
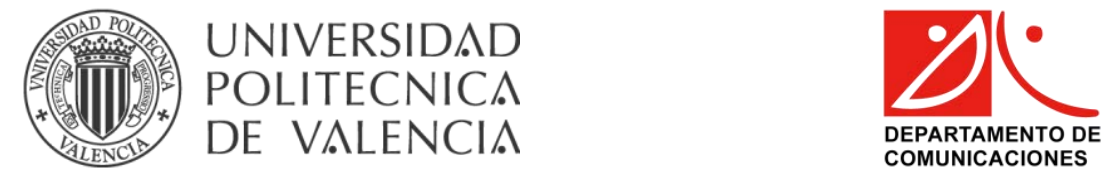

Thesis for the degree of Doctor of Philosophy

\title{
Optical processing in the microwave and terahertz regions
}

\author{
Jesús Palací López
}

Supervisor:

Borja Vidal Rodríguez

Valencia, Spain 2013 



\section{Agradecimientos}

Este libro recoge los frutos de una labor de investigación desarrollada durante varios años y a la cual han contribuido muchísimas personas. Es por ello que, aunque espero no olvidar a nadie, estoy seguro de que así será por lo que pido perdón de antemano.

Antes de nada quiero expresar mi gratitud hacia Javier Martí, como director del Centro de Tecnología Nanofotónica, por haberme dado la oportunidad de realizar la tesis doctoral en su grupo de investigación. Muchas gracias también a Borja Vidal por haberla dirigido, sobre todo por su apoyo constante y gran compañerismo. También a Alex, mi colega en la línea de THz. Mucho ánimo, ya verás como todo sale bien.

Muchas gracias a todos los compañeros con los que he tenido la suerte de compartir estos años. En especial a Guillermo y Jordaco por los zillones de risas compartidas y por la ingesta desmesurada de alimentos poco saludables. Cómo olvidar la amable gruñonería de Mercé, las locas discusiones científicas con Carlos, el humor del inmutable Rubén, al simpático Javi aguantando de todo con una sonrisa, a Josema recordándonos que no ha almorzado aún, o la alegre risa de Pak. Muchas gracias también al resto de personas del área. A Marta por su amabilidad y ayuda, a Ruth por su buen humor, a María por las divertidas luchas por dispositivos y al reciente Eloi por su extroversión. También a Joaquín por su sonrisa y fruta fresca, y a Sarita por las anchoas. Muchas gracias a Pablo, Antoine, Anita (bellotera) y Mariam por toda la ayuda prestada.

También a toda la gente "del correr": Dani, Carlos, Jose, Amadeu, Luis, Gianni y Satur. Y a la "del comer": a Juan por sus deliciosos platos, y a María y Nuria por sus historias y sentido del humor. También a los profes de la segunda planta, siempre dispuestos a discutir cualquier idea: Roberto, Jaime y Alex. Mucha suerte y felicidad para los quasi-papás David y Clara. Y gracias a todas las personas de secretaría y RRHH que han ayudado tanto estos años: Susana, Caterina e Isabel. 
Imposible olvidar a todos los que han pasado por el NTC, desde Claudio y Verónica hasta Begoña, María, Ingrid y Rubén. Guardo un especial recuerdo de Valentín y Rakesh, con quienes me inicié al mundo de la investigación. Mención de honor para José Caraquitena, un grande que se nos fue.

I am really grateful to Peter Jepsen, who nicely received me in his amazing research group (greetings to Jonas, Krzysztof, Dmitry and Maksim!).

I cannot forget people who provided useful comments during the writing of this thesis. Thanks a lot Nathan, Pablo and Juan Luis!

Finalmente, cómo no agradecer el apoyo y la simpatía de mis amigos. Gracias a Gayard y Almu (aunque se vayan a casar), a Poyastre por su humor del internet, a Carlos por su amistad durante tantos años, a Salva por haserme reír con su asento y a Toni por liarla de vez en cuando para que no nos aburramos.

También a mi familia por su incondicional apoyo. En especial a mis iaios Gonzalo y Paquita, quienes aguantaron dos horas y media de lectura de tesis en idioma ajeno sin dar apenas cabezadas. A Hortensia también por venir con ellos. Al tito Juanjo, la tía Beatriz y a mi primo Ale. A Javi por ser un gran amigo durante tanto tiempo y a Carmen por sus carcajadas capaces de romper cristales.

Finalmente a mis padres. Ellos me han criado y educado y lo poco que soy se lo debo enteramente a ellos. También a mi hermano, porque la vida sería mucho más aburrida sin él. Y a Chancla, mi perro de un palmo favorito. 


\section{Table of contents}

Abstract iii

List of papers vii

Abbreviations used in the text $\quad x i$

1 Introduction 1

1.1 Fiber-optics Systems ..................................................... 3

1.1.1 Optical Fibers ….................................................. 5

1.1.2 Electro-optical Modulation ..................................... $\quad 12$

1.1.3 Photodetection ....................................................... 14

1.2 Microwave Photonics ........................................................... 16

1.2.1 Microwave Photonics Filters ................................... 17

1.3 Terahertz Photonics ....................................................... 20

1.3.1 Terahertz Generation ............................................ $\quad 20$

1.3.2 Terahertz Detection ................................................ 21

1.3.3 Terahertz Photonics in the Telecom Band ............ 23

1.3.4 Terahertz Time-Domain Spectroscopy Systems .... 25

1.4 Motivation and Outline ................................................... 27

$2 \quad$ Photonic Microwave Filtering $\quad 29$

2.1 Filter Response Reconfigurability through Four-Wave Mixing .................................................................................... 30

2.2 Single Band Pass Response based on a Phase-Shifted Fiber Bragg Grating ................................................................... 32

2.3 Single Band Pass Filtering based on Phase Modulation and Microring Resonators 
$3 \quad$ Fiber-based Terahertz Time-Domain Spectroscopy Systems

3.1 Enhancing Spectral Brightness in the Terahertz Band... 40

3.1.1 Terahertz Shaping based on Third-Order Dispersion and Self-Phase Modulation .............................. 44

3.1.2 Terahertz Shaping based on Time-Domain Modulation of the Optical Spectrum 44

3.2 Towards Reduced Acquisition Times

3.2.1 Pulse Delay based on Gain Saturation in Semiconductor Optical Amplifiers

3.2.2 Optical Delay Line based on Frequency-to-Time Modulation

4 General discussion

5 Conclusion 55

References

59

Papers A-G 


\section{Abstract}

The thesis focuses on signal processing in the microwave and terahertz regions by using optical devices operating in the telecommunication band. The limitations of processing in these bands employing conventional technology can be circumvented using optical technology. On one hand, microwave electronics have high losses and are bandwidth-limited. In this case fiberoptics technology provides advantages such as low losses and almost unlimited bandwidth. On the other hand, processing of terahertz signals has been traditionally performed using free-space elements which are bulky and show long-term stability problems. Thanks to the recent development of terahertz sources and detectors compatible with $1.5 \mu \mathrm{m}$ light processing can be performed using fiber-compatible devices, allowing for compact and stable processing systems. The aim of this thesis is the development of fiber optic solutions to address limitations in the processing of signals in the lower part of the electromagnetic spectrum from radio to THz. In particular, novel photonic microwave filter structures as well as fiber optic processing for terahertz spectroscopy are analyzed.

In the field of photonic processing of microwave signals, several new architectures have been studied. Cascaded four-wave mixing is employed to increase the number of taps of finite-impulse response filters based on dispersion. Non-periodic bandpass responses that are necessary for radio frequency applications are also implemented by electrical sampling of narrow optical filters such as phase-shifted fiber Bragg gratings and silicon microring resonators.

With respect to the processing of terahertz signals, techniques to obtain locally increased power density are proposed. One relies on the non-linear distribution of ultrashort pulses through optical fiber while the other is based on the time modulation of the optical source spectrum by means of dispersion and a semiconductor optical amplifier interferometric structure. The increase of generated terahertz power due to the combined effect of sources with enhanced efficiency and optical processing techniques is expected to extend the applicability of tabletop terahertz systems. Finally, the fabrication of optical delays by means of fibre-based components to replace slow delay lines based on mirrors and translation stages is addressed. The solutions proposed are based on the saturation of a semiconductor optical amplifier and single sideband carrier suppressed modulation of the spectrum. While the first approach does not introduce pulse degradation the obtained delays are small, as opposed to the second approach which obtains large delays at expenses of pulse widening. 


\section{Resumen}

El objetivo de la tesis es el procesado de señales en las bandas de microondas y terahercios mediante dispositivos ópticos operando en la banda de comunicaciones. El procesado mediante tecnología convencional presenta una serie de limitaciones que la tecnología óptica permite solventar. Por un lado, los dispositivos electrónicos de microondas tienen pérdidas considerables y están limitados en ancho de banda. En este caso la tecnología de fibra óptica proporciona ventajas en términos de bajas pérdidas y ancho de banda prácticamente ilimitado. Por otro lado, el procesado de señales de terahercios se ha llevado a cabo tradicionalmente mediante elementos en espacio libre con los problemas de tamaño y estabilidad que ello implica. Gracias al reciente desarrollo de generadores y detectores de terahercios alimentados por luz a $1.55 \mu \mathrm{m}$ el procesado puede llevarse a cabo utilizando tecnología óptica, lo que proporciona sistemas de procesado más compactos y estables.

La tesis se centra en el desarrollo de arquitecturas basadas en fibra que solventen las limitaciones actuales del procesado de señales cuyas frecuencias se sitúan entre las bandas de radio y THz. En el área de procesado fotónico de señales de microondas se estudian diversas arquitecturas. Se propone la aplicación del efecto de mezclado de cuatro ondas en cascada como una manera de incrementar el número de coeficientes de filtros de respuesta finita basados en dispersión. También se proponen implementaciones de filtros pasobanda no periódicos útiles en aplicaciones de radiofrecuencia basados en la impresión de filtros ópticos en el dominio eléctrico. En un caso se utiliza una red de Bragg en fibra con un desfase sintonizable en su estructura periódica mientras que en el otro se usa un micro anillo resonante fabricado en silicio.

En cuanto al procesado de señales de terahercios se proponen técnicas para aumentar localmente la densidad espectral de potencia. Una se basa en la distribución no lineal de pulsos ultracortos por fibra óptica mientras que la otra modula el espectro de la fuente óptica en el dominio temporal mediante dispersión y una estructura interferométrica de amplificadores ópticos de semiconductor. Se espera que el aumento de la potencia de terahercios generada, tanto mediante fuentes más eficientes como mediante procesado óptico, permita utilizar estos sistemas para llevar a cabo espectroscopía no lineal y detección a distancia. Finalmente, también se estudia la generación de retardos ópticos con el objetivo de sustituir las lentas líneas de retardo basadas en espejos y etapas de traslación motorizadas que se utilizan habitualmente. Las soluciones propuestas se basan en saturación de un amplificador óptico de semiconductor así como en la modulación banda lateral única con portadora suprimida del espectro de los pulsos. La primera solución proporciona retardos pequeños aunque es escalable y no ensancha los pulsos de femtosegundos, mientras que la segunda consigue retardos considerables a cambio de ensanchar los pulsos debido a la dispersión de tercer orden de la fibra. 


\section{Resum}

L'objectiu de la tesi és el processat de senyals en les bandes de microones i terahercis mitjançant dispositius òptics operant en la banda de comunicacions. El processat mitjançant tecnologia convencional presenta una sèrie de limitacions que la tecnologia òptica permet resoldre. Per una banda, els dispositius electrònics de microones tenen pèrdues considerables i estan limitats en ample de banda. En aquest cas la tecnologia de fibra òptica proporciona avantatges en termes de baixes pèrdues i ample de banda pràcticament il - limitat. Per altra banda, el processat de senyals de terahercis s'ha dut a terme tradicionalment mitjançant elements en espai lliure amb els problemes de grandària i estabilitat que això implica. Gràcies al recent desenvolupament de generadores i detectores de terahercis alimentat per llum a $1.55 \mu \mathrm{m}$ el processat pot portar-se a terme utilitzant tecnologia òptica, la qual cosa proporciona sistemes de processat més compactes i estables.

La tesi es centra en el filtrat de senyals de microones així com en el processat de polsos òptics de femtosegons tant per a incrementar la densitat espectral de radiació de terahercis com per a implementar línies de retard. Es proposa l'aplicació de l'efecte de mesclat de quatre ones en cascada com una manera d'incrementar el nombre de coeficients de filtres de resposta finita basats en dispersió. També es proposen implementacions de filtres passabanda no periòdics útils en aplicacions de radiofreqüència basats en la impressió de filtres òptics en el domini elèctric. En un cas s'utilitza una xarxa de Bragg en fibra amb un desfasament sintonitzable en la seua estructura periòdica mentre que en l'altre s'empra un micro anell ressonant fabricat en silici.

En quant al processat de senyals de terahercis es proposen tècniques per a augmentar localment la densitat espectral de potència. Una es basa en la distribució no lineal de polsos ultracurts per fibra òptica mentre que l'altra modula l'espectre de la font òptica en el domini temporal mitjançant dispersió i una estructura interferomètrica d'amplificadores òptics de semiconductor. S'espera que l'augment de la potència de terahercis generada, tant mitjançant fonts més eficients com mitjançant processat òptic, permet utilitzar estos sistemes per portar a cap espectroscòpia no lineal i detecció a distància.

Finalment, també s'estudia la generació de retards òptics amb l'objectiu de substituir les línies de retard basades en espills i etapes de translació motoritzats que s'utilitzen habitualment. Les solucions proposades es basen en saturació d'un amplificador òptic de semiconductor això com en la modulació banda lateral única amb portadora suprimida de l'espectre dels polsos. La primera solució proporciona retards xicotets encara que és escalable i no eixampla els polsos de femtosegons, mentre que la segona aconsegueix retards considerables a canvi d'eixamplar els polsos degut a la dispersió de tercer ordre de la fibra. 


\section{List of papers}

The following papers have been derived from the work of the Thesis:

[A] B. Vidal, J. Palací and J. Capmany, "Reconfigurable Photonic Microwave Filter based on Four-Wave Mixing," IEEE Photon. J., Vol. 4, No. 3, pp. 759-764, June 2012.

[B] J. Palací, P. Pérez-Millán, G. E. Villanueva, J. L. Cruz, M. V. Andrés, J. Martí and B. Vidal, "Tunable Photonic Microwave Filter with Single Bandpass based on a Phase-Shifted Fiber Bragg Grating," IEEE Photon. Technol. Lett., Vol. 22, No. 19, pp. 1467-1469, Oct 2010.

[C] J. Palací, G. E. Villanueva, J. V. Galán, J. Martí and B. Vidal, "Single Bandpass Photonic Microwave Filter based on a Notch Ring Resonator," IEEE Photon. Technol. Lett., Vol. 22, No. 17, pp. 1276-1278, Sept 2010 .

[D] J. Palací and B. Vidal, "Terahertz Radiation Shaping based on ThirdOrder Dispersion and Self-Phase Modulation in Standard Single-Mode Optical Fiber," J Infrared Milli Terahz Waves, Vol. 33, No. 6, pp. 605614, April 2012.

[E] J. Palací, A. Bockelt and B. Vidal, "Terahertz Radiation Shaping based on Optical Spectrum Modulation in the Time Domain," Opt. Express, Vol. 20, No. 21, pp. 23117-23125, Sept 2012.

[F] J. Palací, A. Bockelt and B. Vidal, "SOA-based Optical Processing for Terahertz Time-Domain Spectroscopy," Electron. Lett., Vol. 48, No. 10, pp. 593-594, May 2012.

[G] J. Palací and B. Vidal, "Tunable Optical Delay Line based on SingleSideband Suppressed-Carrier Modulation," IEEE Photon. Technol. Lett., Vol. 25, No. 1, pp. 43-46, Jan 2013. 
Other contributions by the author not included in the Thesis:

[H] R. Sambaraju, J. L. Corral, J. Palací, V. Polo and J. Martí, "Performance Analysis of Photonic Vector Modulation Techniques for Multi-Gb/s Wireless Links," J. Lightwave Technol., Vol. 26, No. 15, pp. $2684-2691,2008$.

[I] R. Sambaraju, J. Palací, V. Polo and J. L. Corral, "Photonic Envelope Detector for Broadband Wireless Signals using a Single Mach-Zehnder Modulator and a Fibre Bragg Grating," in 34th European Conference on Optical Communication. ECOC 2008, Brussels, Belgium, Sept 2008.

[J] R. Sambaraju, J. Palací, R. Alemany, V. Polo and J. L. Corral, "Photonic Vector Demodulation of $2.5 \mathrm{~Gb} / \mathrm{s}$ QAM Modulated Wireless Signals," in IEEE International Topical Meeting on Microwave Photonics. MWP'08, Gold Coast, Australia, Oct 2008.

[K] G. E. Villanueva, P. Pérez-Millán, J. Palací, J. L. Cruz, M. V. Andrés and J. Martí, "Tunable Microwave Signal Generation using DualWavelength DFB Erbium-doped Fiber Laser," in IEEE International Topical Meeting on Microwave Photonics. MWP'09, Valencia, Spain, Oct 2009 .

[L] J. Palací, J. Herrera and J. Martí, "EAM-SOA-based Millimeter-Wave Frequency Up-Conversion for Radio-over-Fiber Applications," in IEEE International Topical Meeting on Microwave Photonics. MWP'09, Valencia, Spain, Oct 2009.

[M] G. E. Villanueva, P. Pérez-Millán, J. Palací, J. L. Cruz, M. V. Andrés and J. Martí, "Dual-Wavelength DFB Erbium-doped Fiber Laser with Tunable Wavelength Spacing," IEEE Photon. Technol. Lett., Vol. 22, No. 4, pp. 254-256, Feb 2010.

[N] G. E. Villanueva, J. Palací, J. L. Cruz, M. V. Andrés, J. Martí and P. Pérez-Millán, "High Frequency Microwave Signal Generation using DualWavelength Emission of Cascaded DFB Fiber Lasers with Wavelength Spacing Tunability," Optics Commun., Vol. 283, No. 24, pp. 5165-5168, 2010 .

[O] G. Serafino, P. Ghelfi, G. E. Villanueva, J. Palací, P. Pérez-Millán, J. Cruz, C. Porzi and A. Bogoni, "Stable Optically Generated RF Signals from a Fibre Mode-Locked Laser," in IEEE 23rd Annual Meeting of the Photonics Society. Denver, Colorado, USA, Nov 2010. 
$[\mathrm{P}]$ J. Palací, G. E. Villanueva and J. Herrera, "EAM-SOA MillimeterWave Frequency Up-Converter for Radio-over-Fiber Applications," Optics Commun., Vol. 284, No. 1, pp. 98-102, 2011.

[Q] G. Serafino, P. Ghelfi, P. Pérez-Millán, G. E. Villanueva, J. Palací, J. L. Cruz and A. Bogoni, "Phase and Amplitude Stability of EHF-band Radar Carriers Generated from an Active Mode-Locked Laser," J. Lightwave Technol., Vol. 29, No. 23, pp. 3551-3559, Dec 2011.

[R] J. Palací and B. Vidal, "Tunable and Reconfigurable Narrow-band THz Generation using Photoconductive Antennas and Chirped-Pulse Mixing," in IEEE International Topical Meeting on Microwave Photonics. MWP'11, Singapore, Singapore, Oct 2011.

[S] J. Palací and B. Vidal, "Distributed THz Transmitter/Receiver based

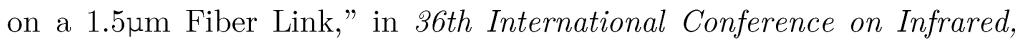
Millimeter and Terahertz Waves. IRMMW-THz 2011, Houston, Texas, Oct 2011.

[T] J. Palací, A. Bockelt and B. Vidal, "Ultra-Short Pulse Shaping based on Time-Domain Spectrum Modulation in a Semiconductor Optical Amplifier," in IEEE International Topical Meeting on Microwave Photonics. MWP'12, Noordwijk, Holland, Sept 2012.

[U] B. Vidal, A. Bockelt and J. Palací, "Cascaded four-wave mixing for microwave photonic harmonic multiplication," IEEE Photon. Technol. Lett., Vol. 25, No. 1, pp. 100-103, Jan 2013. 


\section{Abbreviations used in the text}

APD

ASOPS

CFBG

$\mathrm{CPA}$

$\mathrm{CPM}$

$\mathrm{CW}$

DCF

DFB

DGD

DSF

DSP

EAM

ECL

$\mathrm{EOM}$

FBG

FSR

FWM

GVD

IF

LAPCA

LIA

LT/LTG

MWP
Avalanche photodiode

Asynchronous optical sampling

Chirped FBG

Chirped pulse amplification

Chirped-pulse mixing

Continuous wave

Dispersion compensating fiber

Distributed feedback

Differential group delay

Dispersion-shifted fiber

Digital signal processor

Electro-absorption modulator

External cavity laser

External optical modulator

Fiber Bragg grating

Free spectral range

Four-wave mixing

Group-velocity dispersion

Intermediate frequency

Large-area PCA

Lock-in amplifier

Low-temperature grown

Microwave photonics 
MZM Mach-Zehnder modulator

NLSE Non-linear Schrödinger equation

ODL Optical delay line

PCA Photoconductive antenna

PD Photodetector/photodiode

PMD Polarization-mode dispersion

PMF Polarization maintaining fiber

PSFBG Phase-shifted FBG

QCL Quantum cascade laser

RF Radio frequency

RIN Relative intensity noise

RoF Radio over fiber

SBS Stimulated Brillouin scattering

SLM Spatial light modulator

SoP State of polarization

SPM Self-phase modulation

SRS Stimulated Raman scattering

SSB Single sideband

SSB-SC SSB suppressed carrier

SSMF Standard single-mode fiber

TBP Time-bandwidth product

THz-TDS Terahertz time-domain spectroscopy

TOD Third-order dispersion

TPA Two-photon absorption

TTD True-time delay

UTC-PD Uni-travelling-carrier photodiode

XGM Cross-gain modulation

XPM Cross-phase modulation 


\section{Chapter 1}

\section{Introduction}

Ancient Greeks noticed light travels in straight lines and studied some of its properties such as reflection and refraction. Not much progress was done until $1000 \mathrm{AD}$ when pioneer studies of refraction in lenses and reflections from spherical and parabolic mirrors were carried out by the Arab scholar Alhazan. He was the first to understand vision as the process of light arriving to the eyes. However, the nature of light was not well understood for a long time. Sir Isaac Newton performed his famous experiment in 1665 , proving by means of prisms that white color is made up of all the visible colors. The first discovery of electromagnetic waves out of the visible spectrum occurred in 1800 when infrared light was discovered by the astronomer William Herschel, who theorized the existence of invisible light after splitting light with a prism and finding thermal energy beyond red. This showed that there were unknown ways of radiation. Johann Ritter noticed the existence of rays able to induce certain chemical reactions at the other side of the spectrum. They were called ultraviolet radiation. However, the electromagnetic nature of light was yet to be discovered.

Michael Faraday noticed in 1845 that the polarization of light responded to magnetic fields, and a few years later James Maxwell 
developed equations describing the behavior of electromagnetic waves. The theoretical speed of these waves matched the one measured for light, suggesting its electromagnetic nature. In 1886 Heinrich Hertz generated radio waves in an attempt to prove Maxwell's equations and observed reflection and refraction. He later generated and measured microwaves. The discovery of x-rays and gamma rays in 1895 and 1900, respectively, completed the electromagnetic spectrum we know, shown in Fig. 1.1.

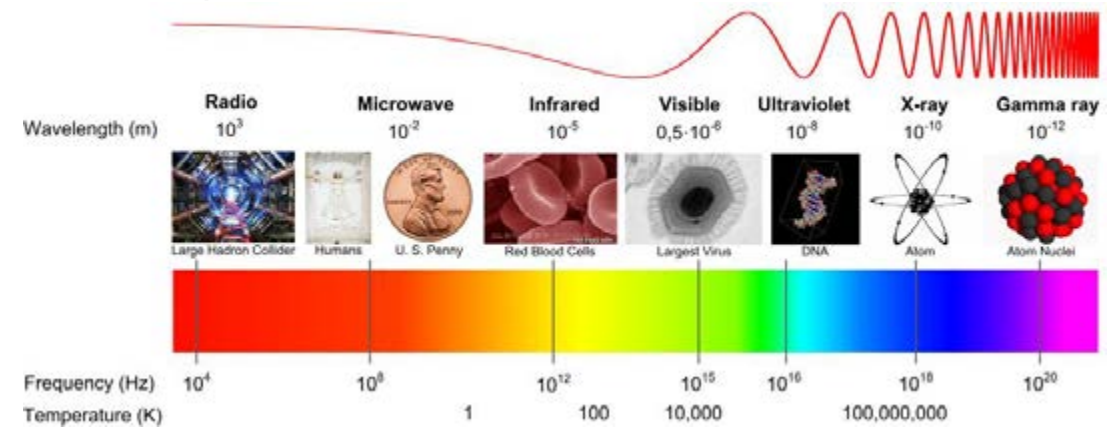

Figure 1.1: The electromagnetic spectrum.

Many applications followed the discovery of the electromagnetic spectrum. It has been exploited for many applications such as broadcasting, data transmission, radar, spectroscopy and imaging and different technologies have been developed for different spectral bands (microwave, submillimeter, optical...). The development of any technology is directly related to its commercial applications. For example, microwave technology advanced fast during the World War II due to interest in developing utilities such as radar, wireless communications and positioning systems. Semiconductor devices were also developed to replace bulky and expensive electron tubes. Similarly, the boom of optical technology came with the development of the laser and optical fiber. Although initial applications focused on data transmission due to the excellent guiding properties of fiber, optical technology has been exploited in processing applications such as sensing [1], imaging [2] and microwave photonics [3].

According to the wavelength we may find interesting features not attainable in other frequency ranges. Optical fiber, for example, is an 
excellent propagation medium with low loss and huge bandwidth. On the contrary, microwave cables show high attenuation, and microwave devices show limited bandwidth. Thus, performing the distribution of wideband microwave signals in the optical domain might be convenient. Although using a region of the electromagnetic spectrum to work with signals in a complete different band can be unnatural, it opens new paths and allows performances not possible with the conventional processing in the same band. Down-conversion of mature optical sources could also provide flexible and reliable light sources in different frequency bands where sources are complicated or immature. The efficient generation and detection of energy in the terahertz band is especially difficult. Acceptable power levels, increased flexibility and processing capability can be obtained by feeding photoconducting materials with optical sources.

This thesis studies techniques to optically process signals in the microwave and terahertz bands using devices developed for the telecommunication band. Microwave photonic filters with comparably enhanced capabilities such as dynamic response reconfigurability and continuous tunability are proposed. The optical processing of ultrashort optical pulses is performed to obtain increased spectral density in the terahertz band as well as develop optical delay lines with potential to reduce the acquisition time of terahertz traces.

\subsection{Fiber-optics Systems}

Lasers are devices that generate and amplify light [4]. The principle of operation of the laser was invented at microwave frequencies, where it was called microwave amplification by stimulated emission of radiation or maser. When we refer to "light" it comprises a huge range of frequencies, from the long infrared region to the X-ray region. Although there is a great variety of lasers all of them share three fundamental elements: a laser medium showing absorption and gain, a pumping process to excite it and feedback elements which determine if the radiation travels through the medium once or it bounces back and forth through the cavity. The first case is employed for the amplification of optical signals while the second is for the generation of radiation through laser oscillation. The latter is shown in Fig. 1.2. 
The first laser was proposed and demonstrated by the beginning of the 60's [5]. Despite its initial lack of utility, intense scientific development in the area led to an uncountable number of applications. Optical fiber provided a suitable medium to guide light and by the end of the 60's GaAs semiconductor lasers operating at room temperature were available [6]. The availability of compact and coherent sources of light along with a low-loss transmission medium led to the development of fiber-optics communications systems able to transmit signals for long distances. Further development of fiber amplifiers by the end of the 80 's contributed to noticeably increase the performance of these systems [7].

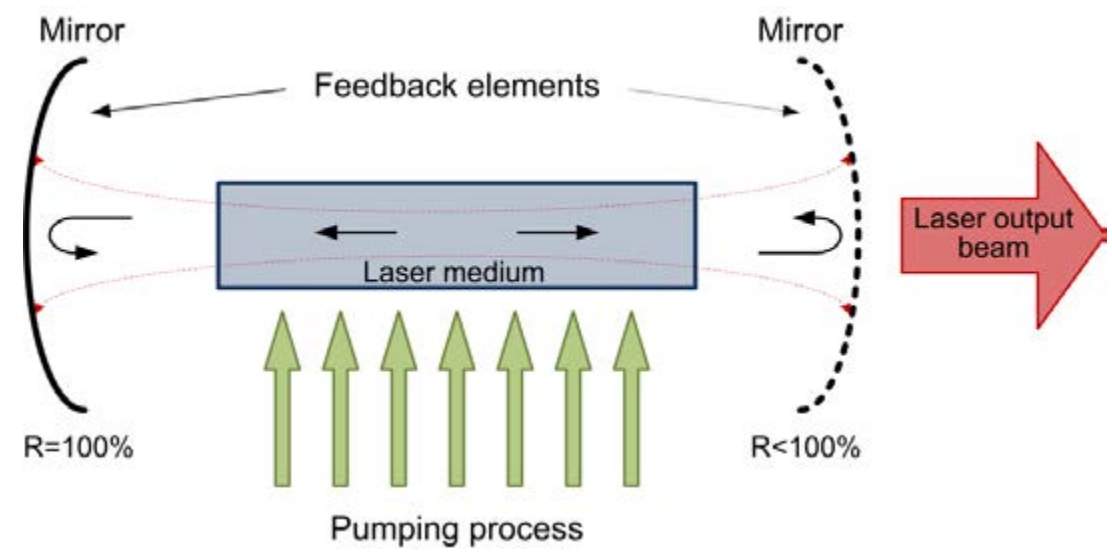

Figure 1.2: Fundamental elements of a laser oscillator.

Although data distribution was first exploited, the optical components developed were soon employed for other applications such as signal processing, sensing, imaging, etc. One of these spin-off applications was the development of the field known as microwave photonics (MWP), which benefits from the low loss and large bandwidth of optical fiber to provide increased performance over traditional microwave systems. Its impact is especially important in communications, radio-over-fiber systems, and signal generation/processing.

A different field where fiber optic technology can provide benefits is beyond the microwave band, in the spectral region known as the 
$\mathrm{THz}$ gap. Different approaches have been proposed to generate radiation in this band and probably optical technology is the most versatile and closer to market. It is mainly implemented using freespace components. However, recent developments allow the use of fiber optic technology to simplify the implementation.

In this section the basic properties of the optical components employed along the thesis are addressed to ease a better understanding of the applications proposed.

\subsubsection{Optical Fibers}

An optical fiber is basically a cylinder made of silica glass which has been covered by a material with lower refractive index. Because of refractive index variations light is guided through the structure. The first glass fibers were fabricated in the 20's [8], although their applicability was very limited up to the 50's when the addition of a cladding led to an important improvement of their guiding capabilities [9]. Their losses were also reduced along the 70 s from $1000 \mathrm{~dB} / \mathrm{km}$ to $0.2 \mathrm{~dB} / \mathrm{km}$ at $1.55 \mu \mathrm{m}$, making this technology commercially attractive [10]. The availability of optical waveguides with such small losses revolutionized the field of optical fiber communications [11]. Although optical fibers were mostly applied for long distance distribution of information, it soon became clear that their dispersive and nonlinear properties could be employed for signal processing [12].

\subsubsection{Geometry and Propagation}

Optical fibers are cylindrical multilayer dielectric structures. Figure 1.3 shows its cross section structure where a core made of silica is covered by a cladding and a jacket, both with lower refractive indexes to confine light. According to the radial variation of the refractive index of the core they can be classified as step-index or graded-index fibers. For the first type there is no variation while for the second one the index decreases for increasing radius, usually in a parabolic fashion. The guiding properties of optical fibers can be observed in a first approximation ${ }^{1}$ by means of a ray picture such as the one shown

\footnotetext{
${ }^{1}$ Assuming the size of the fiber is much larger than the wavelength of the light.
} 
in Fig. 1.3. A ray incident in the center of the core with a certain angle changes its direction of propagation according to the Snell law,

$$
n_{0} \sin \theta_{i}=n_{1} \sin \theta_{r}
$$

The ray bends toward the axial axis and, considering the stepindex fiber scenario, it is guided through the fiber as long as the angle of incidence with respect to the core-cladding interface is large enough to produce total internal reflection. The maximum angle of accepted light depends on the difference in the refractive indexes between the core and the cladding and can be estimated by applying Eq. (1.1) twice.

(a)

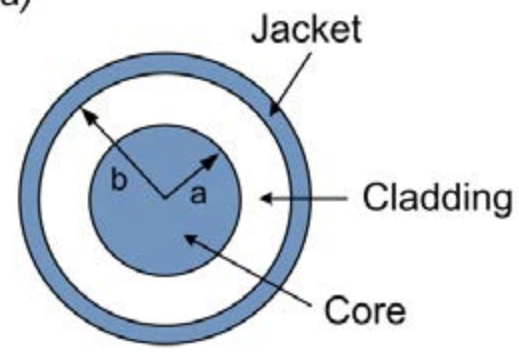

(b)

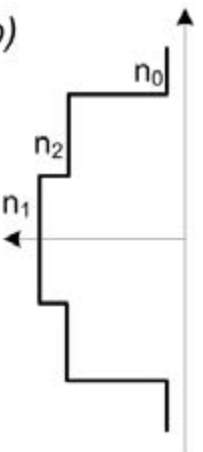

Step-index

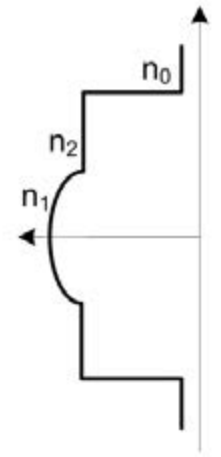

Graded-index

(c)

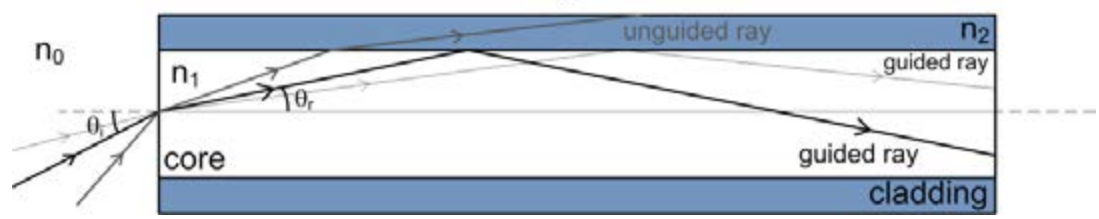

Figure 1.3: (a) Cross section, (b) refractive index profiles, and (c) schematic showing total intern reflection in step-index fibers.

According to Fig. 1.3 there are infinite possible paths for the light propagating through the fiber, which is the origin of multipath dispersion since rays with different angle of incidence arrive at the output of the fiber at a different time. This is especially detrimental for the transmission of pulses, which are considerably broadened thus 
limiting the amount of information that can be transmitted, as well as the reason why grade-index fibers were designed. In these fibers rays with a different angle of incidence propagate at different speeds. Through design of the refractive index profile it is possible to match the distance-index product in such a way that different rays arrive at the same time and multipath dispersion is suppressed.

The performance of optical fibers as transmission medium was further improved by using fibers with radius comparable to the wavelength of the transmitted light. In this case geometrical optics cannot be employed and the Maxwell's equations

$$
\begin{aligned}
& \nabla \times \vec{E}=-\partial \vec{B} / \partial t \\
& \nabla \times \vec{H}=\vec{J}+\partial \vec{D} / \partial t \\
& \nabla \cdot \vec{D}=\vec{\rho}_{f} \\
& \nabla \cdot \vec{B}=0
\end{aligned}
$$

must be solved to analyze propagation. $\vec{E}$ and $\vec{H}$ are electric and magnetic vectors, $\vec{D}$ and $\vec{B}$ correspond to electric and magnetic flux densities, and $\vec{J}$ and $\vec{\rho}_{f}$ are the current density vector and the charge density, respectively.

The constitutive relations

$$
\begin{aligned}
& \vec{D}=\varepsilon_{0} \vec{E}+\vec{P} \\
& \vec{B}=\mu_{0} \vec{H}+\vec{M}
\end{aligned}
$$

relate the flux densities arising due to the fields and the induced electric and magnetic polarizations, $\vec{P}$ and $\vec{M} . \varepsilon_{0}$ and $\mu_{0}$ are the vacuum permittivity and permeability, respectively.

For a nonconducting and nonmagnetic medium such us silica $J=\rho_{f}$ $=\mathrm{M}=0$. Developing Eqs (1.2-1.7) while neglecting attenuation and nonlinear effects and assuming that the index of refraction does not change spatially inside the material leads to the Helmholtz equation [11]

$$
\nabla^{2} \tilde{E}+n^{2}(\omega) \frac{\omega^{2}}{c^{2}} \tilde{E}=0
$$

where $\tilde{E}$ stands for the Fourier transform of the electrical field. 
An optical mode is a term commonly used in optics to refer a specific solution of Eq. (1.8). Optical modes satisfy the boundary conditions imposed by the medium, are uniquely determined by their respective propagation constant $\beta$ and show a spatial profile that does not change with propagation. Although there are several types of modes, fiber optic systems focus on the guided ones.

It is convenient to define the normalized frequency

$$
V=\frac{\omega}{c} a \sqrt{n_{1}^{2}-n_{2}^{2}},
$$

which is related to the number of modes generated in a fiber. High values of $\mathrm{V}$ are desirable because of the reduced attenuation provided by the high confinement of the optical field, although values below 2.405 are necessary to maintain single-mode operation. Typical singlemode fibers propagating signals in the telecom band show core and cladding radius of $4 \mu \mathrm{m}$ and $125 \mu \mathrm{m}$, and indices of refraction of approximately 1.44 and 1.46 for core and cladding, respectively.

\subsubsection{Dispersion in Single-Mode Fibers}

Although single-mode fibers suppress intermodal dispersion, group velocity is usually frequency dependent. Thus, propagating pulses widen because their spectral components travel at different group velocities. This effect is known as intramodal or group-velocity dispersion.

Group-velocity dispersion (GVD) originates from two different sources, material dispersion and waveguide dispersion. The former arises from the dependence of the refractive index on the optical frequency while the latter depends on the physical characteristics of the fiber: radius and index difference. The dimensions of the fiber are usually tailored for the total dispersion to take certain values. Thus, it is possible to shift the dispersion in such a way that the zerowavelength dispersion falls into the range of interest (dispersion shifted fiber or DSF) or to obtain dispersion which is opposite to that of standard fiber for compensation purposes (dispersion compensating fiber or DCF).

It is convenient to model the effect of dispersion by expanding the mode-propagation constant around a given optical frequency $\omega_{0}$, 


$$
\beta(\omega)=n(\omega) \frac{\omega}{c}=\beta_{0}+\beta_{1}\left(\omega-\omega_{0}\right)+\frac{1}{2} \beta_{2}\left(\omega-\omega_{0}\right)^{2}+\frac{1}{6} \beta_{3}\left(\omega-\omega_{0}\right)^{3}+\ldots,
$$

$\beta_{n}$ being the nth-derivative of $\beta$ with respect to the optical frequency, $\omega . \beta_{0}$ is a constant, $\beta_{1}$ is for the propagation of the pulse envelope at the group velocity $n_{g}$ and higher order terms represent dispersion. For standard optical fibers dispersion is mainly given by $\beta_{2}$ and, once it has been compensated, by $\beta_{3}$. Higher order terms are neglected in a first approach although they may become important in long distance dispersioncompensated fiber links.

\subsubsection{Fiber Birefringence}

When ideal single-mode fibers with perfect cylindrical symmetry are considered only one mode exists. However, for real fibers imperfections in the shape of the core and mechanical stress lead to the existence of two orthogonal modes propagating through the fiber. This is known as modal birefringence and is the origin of polarization-mode dispersion (PMD). The propagation constant, and therefore the mode index, is slightly different between signals propagating through the fast and slow axis of the fiber, $x$ and $y$ respectively. The intensity of modal birefringence is given by the difference between mode indices as

$$
B=\left|n_{x}-n_{y}\right| .
$$

For a given $B$ the modes periodically exchange their power as they propagate through fiber. The period of this exchange is given by the beat length parameter,

$$
L_{B}=\lambda / B
$$

However, random changes of the birefringence originating from temperature changes and vibrations result in random coupling between the orthogonal modes. Accordingly, the total contribution of PMD must be treated as a stochastic process. Its effect is often characterized by means of the differential group delay (DGD), which is an estimation of the average time delay between the states of 
polarization ( $\mathrm{SoP}$ ) travelling through the slow and fast axis of the fiber. Although PMD is not especially detrimental for continuous-wave light it degrades transmitted pulses [13]. Different spectral components travel at different speeds according to differences between group velocities, considerably widening optical pulses transmitted through long fiber links. Although the random nature of PMD makes its compensation more difficult, dynamically adaptable compensating systems are currently available.

Fiber birefringence can also be exploited to obtain different functionalities in fiber-optic systems. For example, it has been employed to make couplers, splitter, isolators and optical filters [14] thanks to the development of polarization maintaining fiber (PMF) [15]. This fiber introduces high birefringence by means of non-circular cores which are tailored by means of small stressing pieces as shown in Fig. 1.4. Although there are several types of PMF according to the design of its cross section, PANDA and bow-tie are the most extensively used ones. PMF is able to propagate optical signals with immunity to environmental perturbations as long as the field is linearly polarized and aligned with the principal axis of the fiber.

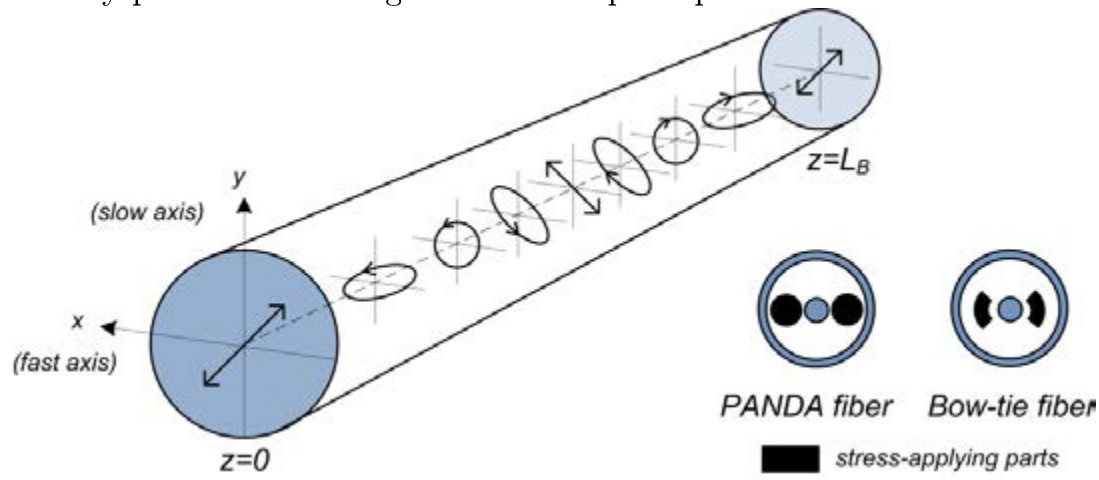

Figure 1.4: State of polarization in a birefringent fiber and cross sections of two types of polarization maintaining fibers.

\subsubsection{Nonlinear Effects}

Nonlinear optical effects are phenomena that arise from the propagation of intense optical fields through a medium [12]. Since the optical field is confined into a small cross section the response of the 
fiber becomes nonlinear due to high power density, affecting propagating signals and generating new optical fields.

Although nonlinear effects are detrimental in transmission systems they are widely used to process light. They can be classified as elastic and inelastic. The former are related to instantaneous changes in the electric polarization $\mathbf{P}$, which lead to changes in the phase and frequency of the signals, while for the latter incident photons are annihilated to create a photon of lower energy and a phonon.

Self-phase modulation (SPM), cross-phase modulation (XPM) and four-wave mixing (FWM) are elastic processes with almost instantaneous response time (below the femtosecond). Stimulated inelastic scattering processes such as Raman and Brillouin, however, are much slower. Stimulated Raman scattering (SRS) and stimulated Brillouin scattering (SBS) take place in a tenth of picosecond and nanosecond timescales, respectively. The SBS effect can be neglected when working with ultra short pulses.

Elastic processes are modeled through the nonlinear permittivity which leads to an intensity dependent refractive index,

$$
\tilde{n}=n+n_{2}|E|^{2},
$$

$n_{2}$ being the nonlinear index coefficient.

Equation (1.8) can be further developed including Eqs. (1.10,1.13). In the Thesis continuous waves of low power or optical pulses of the order of femtoseconds are going to be studied. Under these conditions the nonlinear Schrödinger equation (NLSE) for propagation of pulses through optical fiber is obtained [12]:

$$
\frac{\partial A}{\partial z}+\frac{\alpha}{2} A+i \frac{\beta_{2}}{2} \frac{\partial^{2} A}{\partial T^{2}}-\frac{\beta_{3}}{6} \frac{\partial^{3} A}{\partial T^{3}}=i \gamma\left(|A|^{2} A+\frac{i}{\omega_{0}} \frac{\partial}{\partial T}\left(|A|^{2} A\right)-T_{R} A \frac{\partial|A|^{2}}{\partial T}\right)
$$

The left part of the equation is for attenuation and dispersion while the right one represents nonlinear effects ${ }^{2}$. $A$ is for the slowly varying pulse envelope, $\omega_{0}$ is frequency, $T_{R}$ is for the self-frequency shift induced by intrapulse Raman scattering, $T$ is a time variable

${ }^{2}$ From left to right: self-phase modulation, self-steeping and the Raman effect. 
which takes the position of the pulse propagating along the z-axis as reference and $\gamma$ is the fiber nonlinear parameter defined as

$$
\gamma=n_{2} \omega_{0} / c A_{e f f}
$$

$\mathrm{A}_{\text {eff }}$ being the effective core area which is approximately $\pi \mathrm{w}^{2}$, and $\mathrm{w}$ the width of the fundamental mode propagating through the fiber which depends on the core radius and core-cladding index difference.

According to the presented parameters Eq. (1.14) can be numerically solved to obtain the combined effect of dispersion and nonlinearities on the pulse propagating through optical fiber.

\subsubsection{Electro-optical Modulation}

Semiconductor lasers can be directly modulated through their bias current. To modulate other types of lasers the external modulation of the generated field becomes necessary. External modulation is appealing even for semiconductor lasers because it allows high frequency modulation not attainable by direct modulation while improving the performance of the photonic link through increased linearity. External optical modulators (EOMs) are classified according to the parameter of the optical signal that is modulated. Phase modulators are commonly based on the Pockel's effect of electro-optic crystals such as lithium niobate $\left(\mathrm{LiNbO}_{3}\right)$ while amplitude modulation is often performed by means of interferometric structures (MachZehnder and Michelson) or materials with controllable absorption (electro-absorption modulators, EAM).

Figure 1.5 shows the phase modulation of an optical signal in a $\mathrm{LiNbO}_{3}$ crystal, which is widely used for modulators in the telecom band. When an electrical field is applied to the electrodes a change in the refractive index of the crystal is induced leading to variations in the phase of the optical signal due to differences in the effective propagation length. Due to the crystalline structure of $\mathrm{LiNbO}_{3}$ the phase modulation is more efficient for a certain optical axis of the crystal. Accordingly the use of polarization controllers is recommended for increased modulation efficiency. 
The Mach-Zehnder modulator (MZM) is widely employed for amplitude modulation. It is based on a Mach-Zehnder interferometer where the phase modulation performed on one or both arms is converted into amplitude modulation. The modulation voltage applied to the modulator is usually comprised of constant and time-varying components, $V_{\text {bias }}$ and $V_{\text {mod }}(t)$, respectively. $V_{\text {bias }}$ is chosen to operate the modulator in different points of its transfer function according to the particular application. For example, data modulation requires linear responses and quadrature biasing (QB) is needed. On the other hand, for nonlinear applications, maximum or minimum transmission biasing (MaTB and MiTB, respectively) provide better performance. Figure 1.6 shows the structure of a MZM as well as its transfer function. The half-wave voltage $\left(V_{\pi}\right)$, this is the voltage required to induce a $\pi$ phase shift between the arms of the interferometer, is a quality parameter of the modulator since it is related to the efficiency of the optical modulation. Small values of $\mathrm{V}_{\pi}$ are desired for increased efficiency.

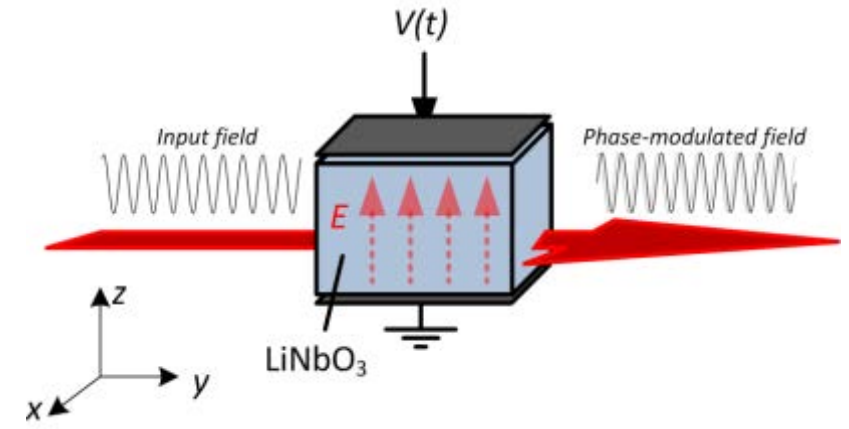

Figure 1.5: Electro-optical phase modulation.
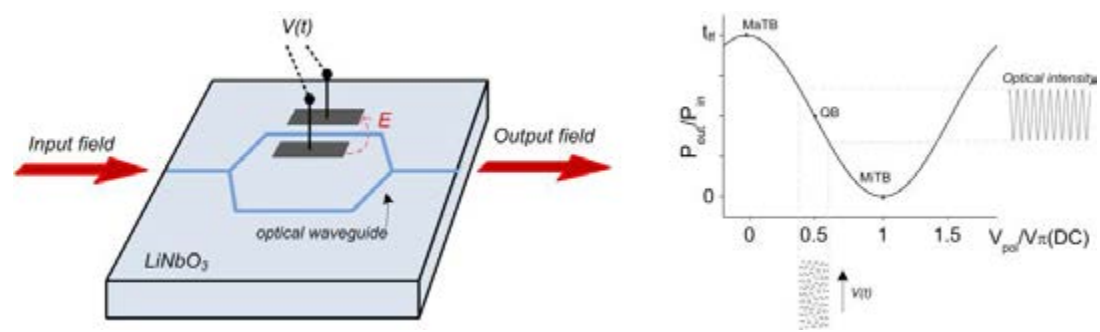

Figure 1.6: Schematic of a Mach-Zehnder modulator and its transfer function. 
For the modulating voltage

$$
V(t)=V_{\text {bias }}+V_{\text {mod }}(t)=V_{\pi}\left(V_{\text {pol }}+m \cdot \cos \left(\omega_{\text {LO }} t\right)\right),
$$

$V_{\text {pol }}$ and $m$ being the normalized biasing and modulating amplitudes, respectively, and $\omega_{L O}$ the modulating frequency coming from a local oscillator, the optical field at the output of the MZM is given by

$$
E(t)=\sqrt{2 \mathrm{P} t_{f f}} \cos \left(\frac{\pi}{2}\left(V_{p o l}+m \cdot \cos \left(\omega_{L O} t\right)\right)\right) \cos \left(v_{0}\right),
$$

where $P$ stands for the optical power at the input of the MZM, $t_{f f}$ for insertion losses and $v_{0}$ for the frequency of the optical carrier.

Equation (1.15) can be expressed in terms of Bessel functions as

$$
\begin{aligned}
& E(t) \propto J_{0}\left(m \frac{\pi}{2}\right) \cos \left(V_{\text {pol }} \frac{\pi}{2}\right) \cos \left(v_{0} t\right)-J_{1}\left(m \frac{\pi}{2}\right) \sin \left(V_{\text {pol }} \frac{\pi}{2}\right) \cos \left(\left(v_{0}-\omega_{L O}\right) t\right) \\
& -J_{1}\left(m \frac{\pi}{2}\right) \sin \left(V_{p o l} \frac{\pi}{2}\right) \cos \left(\left(v_{0}+\omega_{L O}\right) t\right)-J_{2}\left(m \frac{\pi}{2}\right) \cos \left(V_{p o l} \frac{\pi}{2}\right) \cos \left(\left(v_{0}-2 \omega_{L O}\right) t\right) \\
& -J_{2}\left(m \frac{\pi}{2}\right) \cos \left(V_{p o l} \frac{\pi}{2}\right) \cos \left(\left(v_{0}+2 \omega_{L O}\right) t\right)+J_{3}\left(m \frac{\pi}{2}\right) \sin \left(V_{p o l} \frac{\pi}{2}\right) \cos \left(\left(v_{0}-3 \omega_{L O}\right) t\right) \\
& +J_{3}\left(m \frac{\pi}{2}\right) \sin \left(V_{\text {pol }} \frac{\pi}{2}\right) \cos \left(\left(v_{0}+3 \omega_{L O}\right) t\right) \ldots
\end{aligned}
$$

where $J_{n}(\cdot)$ is the $n$-th order Bessel function of the first kind. As observed from Eq. (1.18) several frequencies arise from the singlefrequency modulation of the laser due to the nonlinear response of the MZM. The amplitude of the components is given by the Bessel functions depending on the index of modulation of the MZM and by sinusoidal terms depending on the bias point. Thus, it is possible to employ the modulator for linear or nonlinear operation by controlling the value of these parameters.

\subsubsection{Photodetection}

Being able to recover electrical signals which have been optically processed and/or transmitted is fundamental for fiber optics systems. The term photodetector (PD) refers to optoelectronic devices which absorb optical energy to generate electrical signals. Ideal PDs should have high sensitivity, low noise and fast response. Compatibility with optical fibers is also required, meaning that their size must be similar 
to that of the core of fiber. All these requirements are fulfilled by semiconductor-based PDs.

Optical absorption is the fundamental mechanism behind the photodetection process. The simplest photodetector consists of a semiconductor slab in which incident photons with energy exceeding the bandgap of the material generate electron-hole pairs. GaAs is employed for the $800 \mathrm{~nm}$ region while $\mathrm{Si}$ is suitable at $1 \mu \mathrm{m}$ and InGaAs compounds are used for the telecom band of $1.5 \mu \mathrm{m}$. As long as an electrical field is applied to the material the accelerated electrons and holes originate an electric current and the signal is recovered. Important parameters defining the performance of a PD are responsivity, bandwidth and dark current. Responsivity measures how efficient the photodetector is in terms of generated current per incident optical power. The bandwidth of the PD is given by the speed at which it responds to variations in the optical power. It should be fast enough to resolve the optical waveform of interest, which for high-bandwidth communication applications implies bandwidths of several tens of $\mathrm{GHz}$. The bandwidth depends on the time required by charges to travel to the electrodes and also on the response time of the electrical circuit in charge of processing the measured current. The former depends on the width of the junction while the latter depends on both the capacitance of the PD and the time constant of the receiver system. Accordingly, there is a trade-off between short transit times and high junction capacitances that ultimately limits the detection bandwidth. The dark current, which is the current generated in the absence of optical power, is the last important parameter of a $\mathrm{PD}$ and its value should be negligible.

There are several types of photodiodes. The most common is the p-i-n PD, which consists of an intrinsic layer placed between p- and ndoped layers. Good performance is provided by these structures despite the existent trade-off between high responsivity and fast response. This trade-off arises from saturation of the current density in the junction. Although wider junction areas may provide increased output current the bandwidth lowers. Avalanche photodiodes (APD) are suitable for low optical power operation because they provide larger responsivities thanks to a gain layer which generates additional 
electron-hole pairs through impact ionization. New designs of the epitaxial layers such as the uni-travelling-carrier photodiode (UTCPD) have been proposed for increased bandwidth. In the UTC-PD [16] the absorption takes place in the p-layer, not in the intrinsic one. Holes transport does not limit the bandwidth of the device as opposed to $\mathrm{p}-\mathrm{i}-\mathrm{n}$ PDs because pair electron-holes are generated in the p-layer. This allows for faster responses limited by the velocity of the electrons. This device has shown operation at speeds exceeding $1 \mathrm{THz}$ [17] with high linearity suitable for data generation. Higher frequencies are attainable by means of photoconductive materials with subpicosecond responses such as the ones employed in photoconductive antennas.

\subsection{Microwave Photonics}

The development of optical devices described in the previous section originated the field of microwave photonics. This interdisciplinary field benefits from the complementary features that optical technology can provide to microwave signals. Motivation on MWP research is justified by the capability of optical devices to distribute signals over long distances and process huge spectral bandwidths.

The first MWP systems were employed for optical distribution of electrical signals as shown in Fig. 1.7. An electrooptical device modulates the electrical signal onto one, or more, optical carriers which are distributed through an optical medium (usually fiber) before being recovered in an optoelectronic converter. Such a system is known as a photonic microwave link. Its main advantages come from the medium properties: low distortion and attenuation which allows the distribution of signals for hundreds of kilometers with acceptable degradation; independence of data format, which means that baseband and radio frequency ( $R F$ ) signals can be transmitted with equal performance; low weight and immunity to electromagnetic interferences. This justified the appearance of radio-over-fiber (RoF) systems which perform distribution of radio signals, usually allocated in the microwave and millimeter bands, from a central station (CS) to one or more base station (BS). The electrical signals transmitted can be allocated in baseband with respect to the optical carrier, at an 
intermediate frequency (IF) or directly at the desired radiation band. The best option will depend on the number of BSs although the latter is commonly employed because it allows the use of simple BSs.

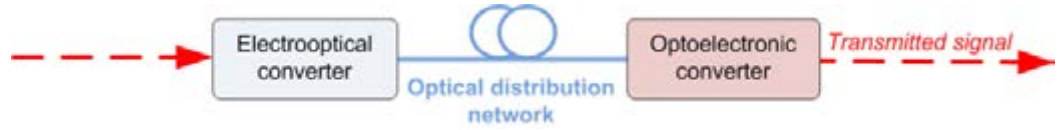

Figure 1.7: Microwave photonic link.

\subsubsection{Microwave Photonic Filters}

MWP technology soon showed potential to perform additional functionalities on electrical signals, not being limited to mere distribution [18]. The application of optical technology to the processing of electrical signals was first proposed in 1976 by Wilner [19]. The optical processing of electrical signals provides the unique capability of processing large bandwidth signals at high frequencies with a performance hardly attainable by means of electrical processing. Moreover, since the processing is performed in the optical domain inefficient conversions between the optical and electrical domains are avoided when signals already injected into an optical distribution system are processed $[20,21]$.

Two different approximations can be taken regarding optical processing: free-space and guided optics. Solutions based on free-space optics use spatial light modulators (SLM) to modify the polarization, phase and/or amplitude of an optical beam as a function of space and time according to an electrical or optical signal which is often twodimensional. The SLM employed in optical processing are usually based on nematic and ferroelectric liquid crystals, electro-optical crystals, photorefractive materials and multiple quantum well layers [22]. Although SLMs are able to perform different kinds of processing with a high degree of parallelism they show problems of stability and dependence with respect to temperature changes and mechanical vibrations. Their bulkiness, high losses and low modulation frequency limit the maximum true-time delay (TTD) they are able to achieve, which is much smaller than the ones obtained in integrated optics systems. Due to these disadvantages the solutions presented in this thesis will focus on guided optics solutions. However, an interested 
reader should check [22-25] for some interesting SLM-based architectures.

The main application fields of the optical processing of electrical signals are analog-digital conversion [26], arbitrary signal generation [27], optical beam-forming [28] and optical filtering [29]. From now on this section focuses on the optical filtering of electrical signals.

In signal processing systems a filter is a device that selects (removes) the wanted (unwanted) components or features from a signal. Signal filtering has been performed by means of baseband digital signal processors (DSPs) [30] or microwave structures as ring resonators, coupled waveguides and impedance mismatching [31] during decades. However, demand for the processing of signals with increasing bandwidth prevents the use of traditional techniques due to several limitations that may be overcome by optical processing techniques.

Microwave photonic filters are photonic subsystems which perform the equivalent tasks of microwave filters but in the optical domain. This alternative approach to microwave filtering offers benefits as spectrally constant loss, electromagnetic immunity, tunability and reconfiguration of the filter response [32]. Thus, photonic filters are able to process wideband signals from low frequencies (a few $\mathrm{MHz}$ ) to the microwave, millimeter and higher bands thanks to the large bandwidth of the optical delay lines available. As opposed to electronic filters, the response of the microwave photonic filters is independent of the electrical frequency because the central frequency of the filter depends exclusively on the optical delay introduced in the structure. Indeed, in practical systems the frequency response is limited by the bandwidth of the electrooptic and optoelectronic converters (modulators and photodiodes, respectively).

Transversal filters similar to the ones employed in digital processing were first implemented. The microwave amplitude transfer function of an N-tap transversal photonic filter assuming optimum state of polarization for the optical signals is given by [33]

$$
\left|H_{R F}(f)\right|=\Re \cos \left(\frac{\beta f^{2}}{2}\right)\left|\sum_{k=1}^{N} P_{k} e^{-j[2 \pi f(k-1) \Delta \tau]}\right|,
$$


$\Re$ being the photo-diode responsivity, $\beta$ the fiber dispersion parameter, $f$ the electrical frequency, $P$ the optical power and $\Delta \tau$ delay. The response obtained is periodic in frequency with a spectral periodicity called free spectral range (FSR). The selectivity, which is the ratio of FSR over the $-3 \mathrm{~dB}$ bandwidth of the main lobe, as well as the relation between the main and secondary lobes are often employed to quantify the performance of filters.
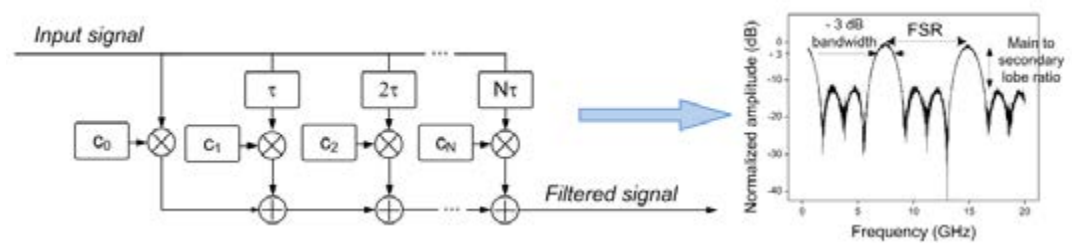

Figure 1.8: Concept of N-tap transversal filter and experimental response of a uniform 4-tap photonic microwave filter.

By using photonic filters it is possible to perform processing of signals in the microwave and millimeter bands in both time and frequency domains. Notch, band pass and high pass responses are examples of spectral filtering, while temporal filtering is performed in coding and decoding, correlation functions and analog-digital or digital-analog conversion [22].

Photonic filters can also be categorized according to their coherence. The coherence of an optical field is related to how an optical signal split and combined again after propagating different distances interfere. Although an ideal monochromatic signal would perfectly interfere because its phase varies with time in a linear fashion, real optical sources are not completely monochromatic and their phase and amplitude show irregular fluctuations at a speed dependent on the line width of the source, $\Delta v$. It is convenient to define the coherence time, $\tau_{c}$, as [34]

$$
\tau_{c}=\frac{1}{4 \pi \Delta v} .
$$

Thus, there is a maximum value for the differential delay of our imaginary interferometer which allows proper interference of the signal. It should be small compared to $\tau_{c \text {. }}$ 
Consequently, coherent or incoherent processing of the signal is performed depending on the delays employed in the implementation of the photonic filter. The main reason to perform coherent filtering is that they allow the implementation of negative taps due to the conservation of phase. However, since the phase of the optical signal is conserved they are very sensitive to vibrations and changes in temperature and polarization [35]. This limits their applicability and promotes the development of incoherent systems, which are less flexible but more robust. Consequently, the filtering schemes proposed in this thesis are to fall into this category.

To sum up, the optical processing of electric signals in the microwave and millimeter bands provides important advantages compared with traditional all-electronic processing, especially in terms of tunability and reconfiguration of the implemented responses. The contribution of this thesis to the development of novel optical filtering structures is presented in Chapter 2.

\subsection{Terahertz Photonics}

The terahertz $(\mathrm{THz})$ band, also referred as submillimeter or terahertz radiation, lies between the microwave and infrared regions of the spectrum. Although there is no standard definition for this band it is commonly accepted that it comprises frequencies from $100 \mathrm{GHz}$ to 10 $\mathrm{THz}$, equivalent to wavelengths from $3 \mathrm{~mm}$ to $30 \mu \mathrm{m}$ or energies between $0.5 \mathrm{meV}$ and $50 \mathrm{meV}$ [36]. The $\mathrm{THz}$ region has long been studied in basic sciences such as astrophysics and molecular chemistry. However, signals falling into this spectral band have not been broadly utilized due to the unavailability of simple and efficient generation and detection processes. This is why this band has been traditionally known as the "THz gap". This section focuses on the different $\mathrm{THz}$ technologies existent with emphasis on photonic-based approaches.

\subsubsection{Terahertz Generation}

Molecular gas lasers such as far-IR and p-type Ge lasers [37, 38], synchrotrons [39], free-electron lasers [40] and back-ward wave oscillators [41] have been employed as bright $\mathrm{THz}$ sources for a long time despite high cost and bulkiness. Technological advances in the 
last decades have led to a wide range of techniques able to generate signals in this frequency band, most of them relying on the frequency conversion of other sources. Techniques based on the up-conversion of microwave signals by means of frequency mixing provide good performance up to a few hundreds of $\mathrm{GHz}$ [42] while solutions based on down-conversion from the infrared band are less limited in frequency and more flexible. Direct generation of signals in the $\mathrm{THz}$ band is also a possibility and the recent development of quantum cascade lasers (QCL) [43] providing high output power is promising although they require cryogenic cooling and the lack of tunability of their emission wavelengths must be addressed. From now on we focus on photonic-based solutions.

Several approaches have been proposed to generate $\mathrm{THz}$ radiation by means of optical sources. They can be grouped in major big categories. One exploits a nonlinear medium in which incident electromagnetic waves undergo frequency conversion while the other relies on the acceleration of carriers. The first group comprises frequency down-conversion techniques performed in a non-linear crystal: optical rectification of femtosecond pulses [44], which generates broadband $\mathrm{THz}$ radiation, and difference frequency generation in which two continuous-wave lasers beat into the crystal to generate a single $\mathrm{THz}$ frequency [45]. Into the second group we find the generation of time-varying currents such as the ones generated in photoconductive antennas (PCA) [46] excited by ultra-short pulses in switching or the photomixing effect when continuous-wave optical sources are employed. Electron accelerators, which are able to generate high broadband and narrowband $\mathrm{THz}$ energies, also fall into this category. In free-electron lasers and backward wave oscillators an electron beam is undulated by a periodic structure, a magnet array and a metal grating, respectively.

\subsubsection{Terahertz Detection}

$\mathrm{THz}$ detection techniques are classified according to whether they are able to recover the $\mathrm{THz}$ field (coherent detection) or only its power (incoherent detection). Coherent schemes are usually necessary to perform spectroscopy measurements when both the amplitude and 
phase of the $\mathrm{THz}$ signals must be resolved to determine both the absorption and dispersion of the sample.

Free-space electro-optic sampling [47] and photoconductive antennas [48] are widespread solutions to measure $\mathrm{THz}$ pulses. In both of them the $\mathrm{THz}$ signal is time sampled using ultra-short pulses. The former is based on the Pockel's effect: the $\mathrm{THz}$ field induces birefringence in a nonlinear optical crystal which is measured by means of an optical probe, while for the latter an optical pulse generates photo carriers which interact with the $\mathrm{THz}$ field. Both schemes require varying the optical delay between the optical pulse and the $\mathrm{THz}$ signal, which is usually performed by means of mirrors and motorized translation stages. A common $\mathrm{THz}$ technique for the coherent generation and detection of waves in this band using femtosecond pulses is THz time-domain spectroscopy (THz-TDS) [49].

Photomixing and heterodyne detection are usually employed to measure continuous wave $\mathrm{THz}$ radiation. The former is usually performed in photo-conductive antennas where both the THz energy and the optical probe made of two continuous wave optical components are focused into the gap of the antenna. The amplitude of the detected current depends on the phase shift between the beating of the two components and the detected $\mathrm{THz}$ component. Thus, by changing the phase of the optical probe the $\mathrm{THz}$ signal is mapped in time [50]. Heterodyne detection works in a similar way. Instead of using two-wavelength optical sources to select the $\mathrm{THz}$ signal to be measured a local oscillator is directly mixed with the submillimeter radiation and an intermediate frequency which amplitude and phase can be measured using conventional electronic devices is obtained. Schottky diodes are often employed as mixers. This approach is usually preferred when sub-THz waves are used.

Several detectors are available if only the intensity of the $\mathrm{THz}$ field is needed (incoherent measurements). Thermal sensors such as Golay cells [51], bolometers [52] or pyroelectric devices [53] are widely used. All of them contain a radiation absorber attached to a heat sink in such a way that incident $\mathrm{THz}$ radiation increases the temperature of the device. The $\mathrm{THz}$ power is estimated through temperature increases. Most bolometers require cryogenic cooling to achieve the 
necessary sensitivity to detect $\mathrm{THz}$ radiation. Pyroelectric detectors use materials that induce an electric polarization as a result of temperature changes. Finally, Golay cells transfer the heat to a small volume of gas where its dilatation pushes a membrane and this change is measured. These detectors respond in a wide bandwidth but are slow compared to other approaches because their principle of operation is based on thermal processes.

\subsubsection{Terahertz Photonics in the Telecom Band}

Optical sources in the band of $800 \mathrm{~nm}$ have been traditionally employed to generate and detect $\mathrm{THz}$ radiation. These sources provide high photon energies which are necessary to excite traditional photoconductive materials such as low temperature grown GaAs (LTG-GaAs). However, sources in the $800 \mathrm{~nm}$ band (usually, Ti:Sapphire lasers) are bulky and expensive.

Extensive research in photonic technology operating in the 1550 $\mathrm{nm}$ band has been carried out during the last decades to allow the deployment of the telecommunication infrastructure needed for the information society. It has led to the development of a wide range of reliable and cost-effective components. Compact and reliable femtosecond lasers [54] are commercially available as well as CW sources such as distributed feedback (DFB) or external cavity lasers (ECL). Other devices developed for the telecom industry include semiconductor- and fiber-based amplifiers and electrooptical modulators. $\mathrm{THz}$ applications could benefit from more compact and cheaper implementations using the optical telecommunication devices at $1550 \mathrm{~nm}$. However, the lack of photoconductive materials with energy band-gaps in the order of the $1550 \mathrm{~nm}$ photon energy, i.e. 0.75 $\mathrm{eV}$, prevented the use of devices in this band for $\mathrm{THz}$ applications.

Frequency-doubling was initially proposed to combine advantages of $1550 \mathrm{~nm}$ sources and $800 \mathrm{~nm}$ materials. However, frequencydoubling schemes add additional complexity and the conversion efficiency is limited, producing low levels of $\mathrm{THz}$ radiation.

Another approach is the development of nonlinear crystals operating in this band. An inorganic semiconductor, GaAs, has been demonstrated to generate and detect $\mathrm{THz}$ signals by means of optical 
rectification of $1550 \mathrm{~nm}$ pulses [55]. However, its electrooptic coefficient is much smaller than that of $800 \mathrm{~nm}$ materials as ZnTe. Organic nonlinear crystals such as DAST [56] are a good alternative because of the high nonlinear optical susceptibilities they show in the telecom band as well as their adaptability to specific applications through molecular design [57].

The design of PC switches compatible with $1550 \mathrm{~nm}$ light has also been addressed. The most common approach focus on the design of devices made of InGaAs or InGaAsP epitaxial layers on InP substrates to obtain reduced band-gap energy. A variety of ultrafast recombination mechanisms based on distributed metallic nanoparticles or deep defect levels have been proposed: As precipitates [58], ErAs nanoparticles [59], Br-irradiated defects [60], Fe-ion-implanted deep levels [61] and InGaAs layers with InAlAs barriers [62]. Although these approaches show the emission of $\mathrm{THz}$ radiation the output power is seriously limited by the critical breakdown field, which happens to be much smaller for these materials than for GaAs.

Mechanisms such as two-photon absorption (TPA) [63], the lateral photo-Dember effect [64] or extrinsic photoconductivity in ErAs:GaAs [65] have been proposed for $\mathrm{THz}$ generation and detection. Although sub-picosecond switching responses were observed by means of TPA, its efficiency was low. On the other hand, the lateral photo-Dember effect shows highly efficient $\mathrm{THz}$ generation in a layer of $\operatorname{In}_{0.53} \mathrm{Ga}_{0.47} \mathrm{As}$ without requiring external biasing of the material. Extrinsic photoconductivity provided impressive performance despite of a limited bandwidth: up to $0.1 \mathrm{~mW}$ of $\mathrm{THz}$ power using a standard femtosecond fiber laser.

Since PCAs operating in the telecommunication band are an excellent alternative to reduce the cost of THz-TDs systems, they were employed for $\mathrm{THz}$ generation and detection in the experiments performed along this thesis. The next section describes the very basics of THz-TDS systems operating with photoconductive antennas. 


\subsubsection{Terahertz Time-Domain Spectroscopy Systems}

THz-TDS systems rely on coherent generation and detection of $\mathrm{THz}$ pulses to determine the real and imaginary parts of the propagation constant, which are necessary to fully characterize materials. The availability of ultra-short optical pulses and the development of semiconductor technology have been essential for the development of THz-TDS systems. The use of ultra-short pulses is important because the bandwidth of the generated $\mathrm{THz}$ spectrum is related to the inverse of its duration. Moreover, since the $\mathrm{THz}$ field is mapped in time the width of the pulses injected to the receiver fixes the minimum temporal step of the traces and thus show a strong influence in the maximum $\mathrm{THz}$ frequency that can be resolved. Obviously, fast materials are required to generate $\mathrm{THz}$ radiation and not only the optical pulses duration but also the material performance limits the bandwidth and dynamic range of the system.

Auston and Lee studied in the 70's the optoelectronic switching of a photoconductive material, technique today known as Auston switching. The first demonstrations used high-resistivity silicon or semi-insulating GaAs $[46,66]$ and optical pulses emitted by a modelocked Nd:glass laser. In the early 80's Smith et al. [67] developed radiation-damaged Si-on-sapphire (RD-SOS), a photoconductive film which was extensively used for a decade due to its fast response. The switching of photoconductive material was initially combined with transmission lines to perform both generation and detection of $\mathrm{THz}$ pulses [68]. However, in 1984 Auston et al. [69] emitted, propagated and coherently detected $\mathrm{THz}$ pulses using the photoconductive material as a Hertzian dipole. It was soon noticed that the bandwidth of the emitted pulse extended into the THz range [70] and, in 1989, van Exter, Fattinger and Grischkowsky showed that this kind of system was useful to perform spectroscopy measurements in the $\mathrm{THz}$ range [49]. Their paper can be considered the origin of THz-TDS systems. In the 90's Ti:sapphire femtosecond lasers emitting $800 \mathrm{~nm}$ pulses with duration below 100 fs became available and the recently developed low-temperature grown GaAs (LT-GaAs) began to be used as the preferred photoconductive material in the implementation of 
PCAs due to the combination of both short carrier lifetime and high mobility [71].

The principle of operation of a photoconductive antenna is quite simple. It is basically an electrical switch which closes when exposed to light. A piece of photoconductive material is therefore required to convert light with photon energy higher than the material bandgap into free carriers. This way a transient of current arises from the free electrons and holes previously generated as long as the material is biased by means of electrodes. The current transient generates an electromagnetic field which is the desired $\mathrm{THz}$ radiation. The switchon time mainly depends on the optical pulse duration, while the switch-off time is determined by the lifetime of the generated free carriers. Short carrier lifetimes are thus important for ultrafast optical switching. Although the processes involved in the detection process are not the same, the principle of operation is similar. The main difference is that instead of being biased, the current introduced by the $\mathrm{THz}$ field "moving" the free carriers is measured.

Figure 1.9 shows the generation of a $\mathrm{THz}$ pulse in a photoconductive antenna. Metallic electrodes which have been previously deposited on the photoconductive material are biased by a continuous voltage. Ultra-short optical pulses delivered through free space are focused between the electrodes, where free carriers close the switch. A transient of current with picosecond duration arises from the movement of the free electrons and holes to the electrode with their opposite charge sign, generating a wideband $\mathrm{THz}$ field. One of the problems with this design is that the THz pulse remains "trapped" in the substrate due to the high difference between the index of refraction of the photoconductive material and air. This problem is partially solved by attaching an optical lens to the substrate. The degradation introduced by the impedance mismatching can be reduced by designing the geometry of a lens whose index of refraction lies between the value of the photoconductive material and the one of free space. Hyper-hemispherical lenses built with high resistivity silicon $(\mathrm{n} \approx 3.42)$ are commonly employed because they avoid internal reflections into the lens and minimize spherical aberration. $\mathrm{A} \mathrm{THz}$ beam of $34^{\circ}$ is expected at the output of this lens [36]. 
Figure 1.10 shows a generic THz-TDS system. Off-axis parabolic mirrors are employed to direct the $\mathrm{THz}$ radiation from the source to the receiver. Set-ups with two mirrors provide a collimated $\mathrm{THz}$ beam while those using four mirrors are able to focus the $\mathrm{THz}$ radiation into a given spatial point. The minimum beam waist of a focused $\mathrm{THz}$ beam, which can be estimated through Gaussian optics, is in the range of a few hundreds of microns. Although the beam waist depends on the $\mathrm{THz}$ frequency certain arrangements of lenses are able to generate frequency-independent beam waists [72].

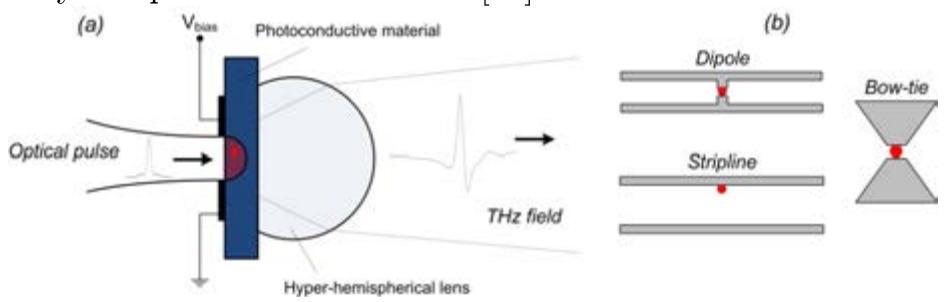

Figure 1.9: (a) Schematic representation of $\mathrm{THz}$ pulse generation in a PCA. (b) Different types of antenna.

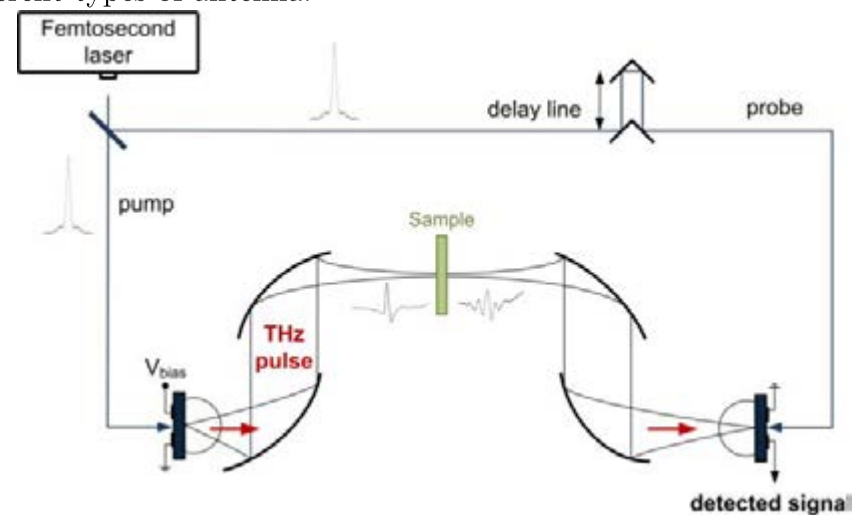

Figure 1.10: THz-pulse emission and detection using photoconductive antennas. Four parabolic mirrors provide a focused $\mathrm{THz}$ beam.

\subsection{Motivation and Outline}

The motivation for this thesis was to investigate optical processing techniques with potential to provide increased performance for signals in the microwave and terahertz bands. Despite the emphasis on demonstrating the feasibility of the proposed techniques by means of experimental work, theory has also been addressed in the form of 
modeling and numerical simulations when required to identify critical parameters of an architecture or confirming the origin of the results.

According to the university regulations, the format for the thesis is a compilation of articles. The thesis is organized as follows. Chapter 1 has presented an introduction which provided a context for the papers and illustrated the very basics of the employed components. Chapter 2 deals with the study of novel photonic microwave filters which provide enhanced capabilities such as reconfigurability and tunability of the response. Chapter 3 is dedicated to the processing of femtosecond pulses to increase both spectral power and acquisition speed in terahertz spectroscopy systems. Chapter 4 provides a general discussion of the results and Chapter 5 sums up the main conclusions of the thesis. 


\section{Chapter 2}

\section{Photonic Microwave Filtering}

Among the different types of photonic microwave filters proposed in the literature [32, 73], transversal filters based on optical delays have attracted considerable interest due to their flexibility and their fast tuning. They allow for scalability and tunability through the control of the number of taps and the optical delay, respectively. Moreover, the incoherent nature of the approach provides stable and robust filter responses. Photonic microwave filters based on wavelength division multiplexed (WDM) signals and dispersive elements have been studied [74]. However, their traditional implementation requires a large number of optical sources to implement complex filter responses, which raises cost and limits their deployment. Several solutions have been proposed to solve this problem, as optical slicing of incoherent sources [75], using comb sources instead of continuous wave ones [76] or combining several sources and detectors [77]. Although the first solution greatly reduces the cost by employing cheap sources, its performance is considerably reduced due to noise. The second one is convenient because of the large number of components generated in optical combs, but separate control of the different taps becomes difficult and costly and the pulsed nature of the filter may prevent its application. The third one reduces the number of components of the 
system but requires a microwave coupler which might reduce the flexibility of the filter in terms of bandwidth.

An additional issue of dispersive-based photonic microwave filters is the periodic nature of the filter response inherent to transversal filters. Several techniques have been proposed to address this issue.

The carrier suppression effect in optical fiber has been employed to remove some of the undesired repetitions in periodic filter responses [78]. However, this approach requires difficult adjustment of the optical source parameters, complicating tuning and reconfigurability of the microwave response.

Schemes based on optical processing in fiber have been proposed [79-81]. However, the implementation can be complex [79] or the use of slicing of broadband sources limits the filter performance [80, 81]. Alternatively some proposals are based on processing using spatial light modulators $[73,82]$ to achieve a larger number of taps with a single component at the cost of using a free-space stage.

Finally, some proposals are based on exploiting optical resonances which suppress or amplify the sidebands of a modulated carrier to perform filtering of the electrical signal [83, 84]. Optical filters are suitable to perform single bandpass filtering of electrical signals through direct mapping of their response because they are not always periodic and those that do show FSRs larger than the bandwidth of most optoelectronic converters.

In this Chapter new architectures for photonic microwave filtering are presented. First, dynamic control over the response of WDM photonic microwave filters by means of the four-wave mixing effect taking place in optical fiber is proposed. Additionally, tunable single bandpass filtering structures based on optical resonances are studied.

\subsection{Filter Response Reconfigurability through Four-Wave Mixing}

Four-wave mixing (FWM) is a parametric process in which the nonlinear processes involved modulate a medium parameter, in this case the refractive index of an optical fiber [12]. In parametric processes a nonlinear polarization characterized by the nonlinear susceptibility is induced in the medium. Second-order susceptibility, 
$\chi^{(2)}$, is responsible for second-order processes such as second-harmonic generation and sum-frequency generation, which are not efficiently generated in optical fiber since silica is an isotropic medium. On the other hand, the third-order susceptibility, $\chi^{(3)}$, is responsible for thirdorder processes such as third-harmonic generation, parametric amplification and FWM which are easily stimulated in nonlinear fibers.

In the general case, FWM generates new waves when three optical carriers of different wavelength and linearly polarized along the same optical axis copropagate through an optical fiber. An induced nonlinear polarization vector is obtained as the product of the thirdorder susceptibility parameter and the optical field. This polarization vector leads to different beatings among the optical carriers which generate new waves whose frequencies are given as a combination of theirs. Phase matching is required for efficient generation of FWM. This is usually obtained through operation near the zero-dispersion wavelength of the fiber. Interested readers may read [12, 85] for a complete analysis of parametric processes. In the nondegenerated case (i.e. two pumps and a signal wave), FWM generates several terms. The weaker ones can be neglected and the frequency of the strongest one, known as the idler wave, is given by,

$$
\omega_{4}=\omega_{1}+\omega_{2}-\omega_{3}
$$

It is also possible to generate idler waves employing only two pump carriers. It must be noticed that any wave with enough power can act as pump. It means that an efficient FWM can generate idler waves which in turn may be powerful enough to stimulate new waves through FWM. This is known as cascaded FWM [86, 87]. Figure 2.1 shows the different FWM scenarios discussed. 

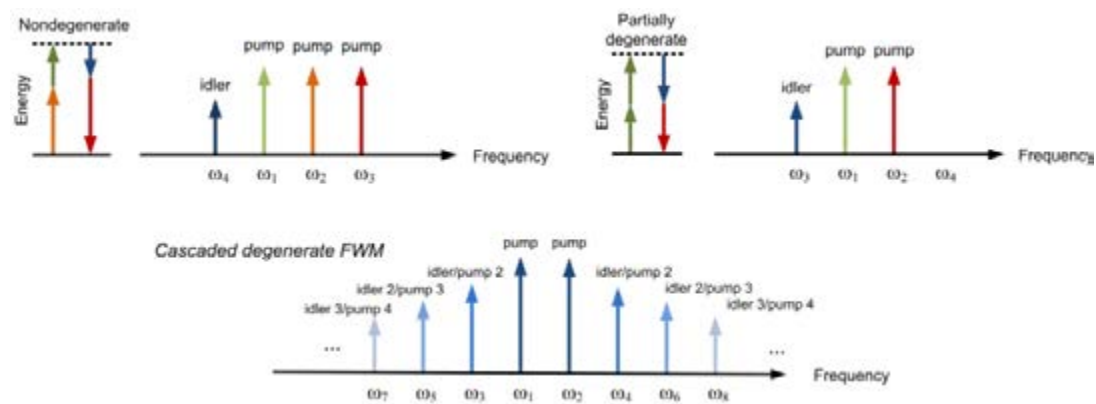

Figure 2.1: Four-wave mixing, degenerate FWM and cascaded degenerate FWM.

Thus, FWM can be exploited for microwave filtering from two perspectives. Firstly, the new generated idler waves are new taps and therefore FWM can be used to reduce the number of components needed to implement a filter of a given number of taps. Secondly, it is possible to control the amplitude distribution of the taps by changing the optical power of two optical carriers propagating through an optical fiber. In WDM photonic filters the bandwidth of the bandpass is directly related to the number of taps/ carriers for a given freespectral range (i.e. for a given delay between taps). A filter based on cascaded FWM should be able to control this bandwidth according to the optical power. Paper A reports experimental measurements showing reconfigurability of the filter response based on FWM. A reel of highly nonlinear fiber was employed for efficient FWM generation and a second reel of standard fiber was used to implement the delays as through chromatic dispersion

$$
\Delta \tau \approx L \cdot D \cdot \Delta \lambda
$$

where D stands for the second order dispersion (SOD) parameter of the $\mathrm{L} \mathrm{km}$ of SSMF and $\Delta \lambda$ is the wavelength difference between the lasers. $\Delta \tau$ is delay as in Eq. (1.19).

\subsection{Single Band Pass Response based on a Phase Shifted Fiber Bragg Grating}

Fiber Bragg gratings (FBG) are widely employed in communication systems due to their simplicity, low insertion losses and compatibility with fiber systems [88]. They consist of a periodic modulation of the 
refractive index of the core of an optical fiber along its longitudinal axis that reflects particular wavelengths and transmits the rest. There are several types of FBG according to different variations in the period of the modulation index, although the simplest configuration is the uniform FBG which shows a constant period along the whole grating. For a period $\Lambda_{\mathrm{B}}$ the reflected wavelength, known as the Bragg wavelength, is given by

$$
\lambda_{\text {Bragg }}=2 n_{\text {eff }} \Lambda_{B}
$$

$\mathrm{n}_{\text {eff }}$ being the effective index of the core mode. For responses in the telecom band, periodicities of approximately half a micron are required. In a practical scenario the grating does not exclusively reflect $\lambda_{\mathrm{B}}$. Due to the finite length of the grating a reflection band whose bandwidth depends on the grating length is obtained. Typical values of 10 to $15 \mathrm{~cm}$ provide reflection bandwidths of $0.2 \mathrm{~nm}$ at $1550 \mathrm{~nm}$.

When a phase shift occurs into the periodic structure of the grating a transmission peak is created into the reflection band. The position of the peak into the band depends on the magnitude of the phase shift. Phase shifts can be introduced during the fabrication process of the grating [89] or externally controlled by means of temperature changes [90], optically controlled variations in the index of refraction [91], magnetostrictive transducers [92] and piezoelectric actuators [93]. Phase-shifted fiber Bragg gratings (PSFBG) have been employed to implement narrow optical filters [89] and dual-wavelength fiber lasers [93]. Paper $\mathbf{B}$ reports a tunable photonic microwave filter based on the mapping of a PSFBG by an optical carrier which has been single-sideband modulated. Because of its simple operation and speed a lead zirconate titanate (PZT) piezoelectric actuator was employed to stretch a piece of uniform FBG, thus providing tunability to the system. Tunable and narrow single-bandpass responses with a bandwidth of $130 \mathrm{MHz}$ and frequencies up to $6 \mathrm{GHz}$ were obtained. The bandwidth of the filter depends on the stretched length, which could be further shortened. Although the tuning range of the system is fundamentally limited by the bandwidth of the FBG it could be extended using chirped gratings. 


\subsection{Single Band Pass Filtering based on Phase Modulation and Microring Resonators}

Optical microresonators have been widely used for different applications such as light generation and amplification, data routing, sensing, switches and logic gates [94]. Microring resonators are structures consistent of a waveguide channeling light in a closed loop. Any loop should be considered, from disks to ellipses, although closed waveguides in the form of rings are often employed. Placing waveguides close to such structure provides access to the structure resonant modes through evanescent coupling, allowing both injection and extraction of resonant wavelengths. They act as optical filters, as shown in Fig. 2.2, where through and drop ports show notch and band pass responses with respect to the input, respectively.
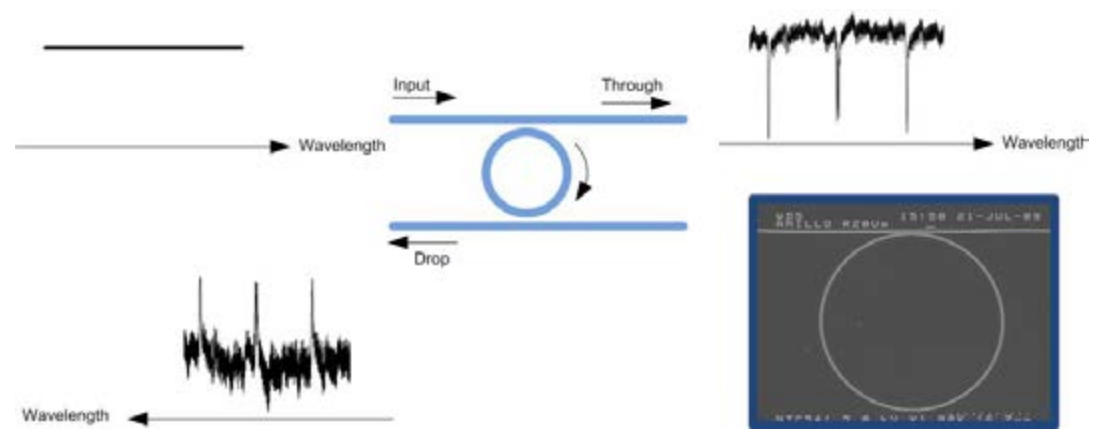

Figure 2.2: Experimental response of a microring resonator. A SEM image is also shown.

Recent efforts are being made towards simpler filtering architectures based on devices that are compatible with complementary metal-oxide-semiconductor (CMOS) silicon-based photonic integration since this technology is expected to reduce cost and size of these structures. Paper $\mathbf{G}$ reports the implementation of a novel tunable single-sideband photonic microwave filter. It is based on phase modulation and a microring resonator whose planar nature is suitable for monolithic integration. Phase-modulated signals show sidebands that are out of phase and result in cancellation of the different terms obtained during photo detection. However, the removal 
of one of the sidebands results in amplitude modulation. We propose sampling of one of the notch responses of a Si ring resonator to obtain microwave band pass filtering from optical notch responses. Due to the large resonator FSR the implemented microwave filter can be considered single band pass. Its response can be tuned using tunable optical sources. 


\section{Chapter 3}

\section{Fiber-based Terahertz Time-Domain Spectroscopy Systems}

As discussed in Section 1.3.4, THz-TDS is a spectroscopic technique which can extract material properties from both the amplitude and phase of the captured radiation. $\mathrm{THz}$ spectrometers are usually implemented using Ti:sapphire sources at $800 \mathrm{~nm}$ and bulk optics which directs the light towards the PCA active area. To maximize the coupling of the optical pulses in the active area, fiber-pigtailed PCA were developed [95]. Packaged PCAs can be obtained by fixing of the aligned optical fiber illuminating the gap and the hiper-hemispherical lens to the substrate, providing compact $\mathrm{THz}$ heads which ease the implementation of THz-TDS systems. An additional step was the development of new materials to allow PCA operating at the telecommunications band. That allows the development of all-fiber THz-TDS instruments [96]. An example of commercially available fiber-coupled THz emitters and receivers is shown in Fig. 3.1 [97]. 


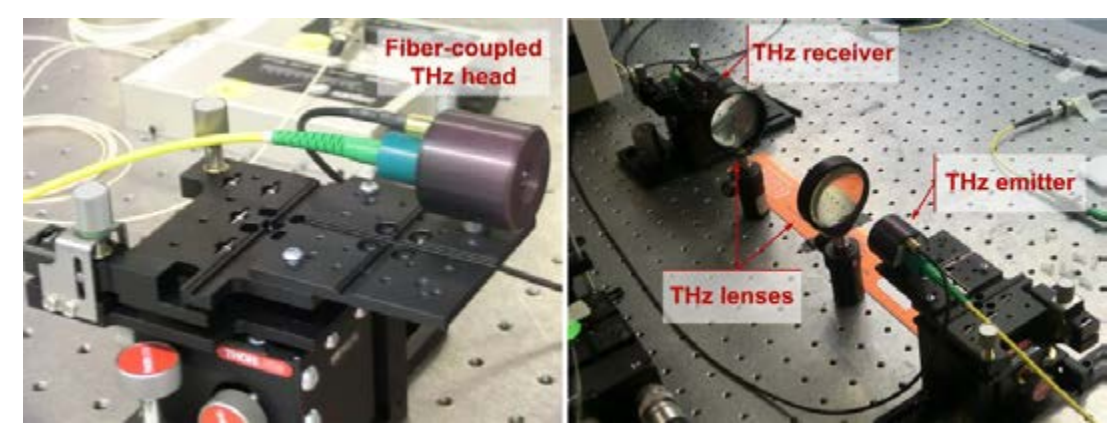

Figure 3.1: Detail of packaged fiber-coupled PCA and THz-TDS system using $\mathrm{THz}$ lenses for beam collimation.

Optical fiber allows the implementation of compact THz-TDS systems, avoids the alignment of the free-space beams of light and results in robust operation with the capability to deploy distributed instruments as well as the reuse of the optical source (the most expensive subsystem) to further reduce the cost of the instrument [98]. However, fiber optics also present implementation issues. As discussed in Chapter 1 optical fiber is a dispersive and non-linear medium. Thus, femtosecond pulses are severely degraded due to their huge bandwidth even for propagation distances of a few centimeters and there is a maximum power that can be handled without degradation [12]. As discussed in [99], dispersion compensation along with careful characterization of the fiber link becomes essential to obtain narrow pulses at the input of the PCAs. However, the matching of the arrival time of the pulses to the input of the PCAs in pump/probe experiments such as THz-TDS spectroscopy is complicated when dispersive media such as optical fiber are employed. Air can be considered neither dispersive nor nonlinear at optics wavelengths. Thus, pulses are not degraded through free-space propagation and their delay can be controlled according to the propagation distance without affecting the performance of the system. However, in fiberbased THz-TDS systems both dispersion and delay management have to be performed simultaneously. Dispersion compensation occurs when

$$
\sum_{i=1}^{n} L_{i} D_{i} \approx 0
$$


$L_{i}$ and $D_{i}$ being length and dispersion of the fiber segments employed in the link.

While for the delay

$$
\frac{\sum_{i=1}^{n} L_{e}^{i} n_{g}^{i}}{c}+\frac{L_{f s}}{c} \approx \frac{\sum_{i=1}^{n} L_{r}^{i} n_{g}^{i}}{c}
$$

must be satisfied. $\mathrm{L}_{\mathrm{e}} \mathrm{L}_{\mathrm{r}}$ and $\mathrm{L}_{\mathrm{fs}}$ are the lengths of the different fibers (standard single-mode fiber or SSMF, DCF, DSF, etc) in the emitter and receiver branches, and that of free-space propagation, respectively. $\mathrm{n}_{\mathrm{g}}$ is for the group indexes of modes propagating in the different types of fiber.

Finding out the combination of optical fibers fulfilling Eqs. (3.13.2) might be not trivial. Moreover, these expressions do not consider nonlinear effects whose effect is important in high-power pulse distribution. However, these equations could be used as an initial approximation before performing experiments.

The setup employed for the measurements performed is shown in Fig. 3.2. Optical pulses with a duration of $100 \mathrm{fs}$ are generated in a passive mode-locked fiber laser at a repetition rate of $50 \mathrm{MHz}$. The optical power is set to values that do not show non-linear degradation in the pulses. No nonlinear degradation up to $5 \mathrm{dBm}$ of average power was observed. DCF was employed to initially time-stretch the optical pulses. Although this type of fiber shows higher nonlinear coefficient due to its comparably smaller core (see Eq. (1.15)) its high $\beta_{2}$ parameter stretches pulses much faster than standard fiber, thus pulses with high peak power travel shorter distances resulting in reduced nonlinear degradation. Pulses are stretched up to the picosecond (papers $\mathbf{D}, \mathbf{F}$ ) or nanosecond range (papers $\mathbf{E}, \mathbf{G}$ ) before being amplified by means of a booster EDFA. Chirped pulse amplification (CPA) is required to obtain enough optical power to operate the PCAs. SSMF is then used to compensate for the dispersion of the fiber link before feeding the antennas. Packaged InGaAs/InAlAs PCAs manufactured and commercialized by Menlo Systems $\mathrm{GmbH}$ are employed to generate and detect $\mathrm{THz}$ radiation. The $\mathrm{THz}$ field is propagated and focused on the receiver gap by using 
$\mathrm{THz}$ lenses (made of polymethylpentene, TPX) before being sampled using a motorized optical delay line (MODL). Phase-sensitive detection at $1 \mathrm{kHz}$ is performed using a lock-in amplifier (LIA) and an optical chopper (OC). $\mathrm{THz}$ traces are recorded in a personal computer using custom Labview-based software. Figure 3.3 shows a recorded pulse as well as its spectrum.

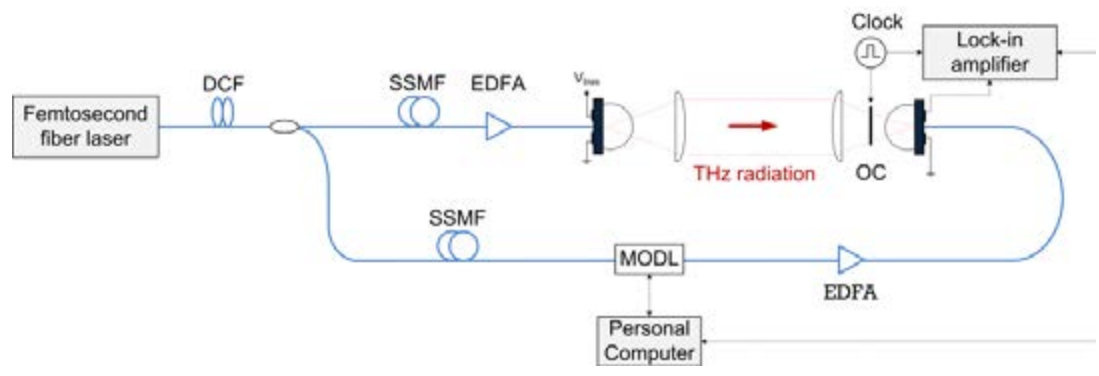

Figure 3.2: Fiber-based THz-TDS system used during the thesis.
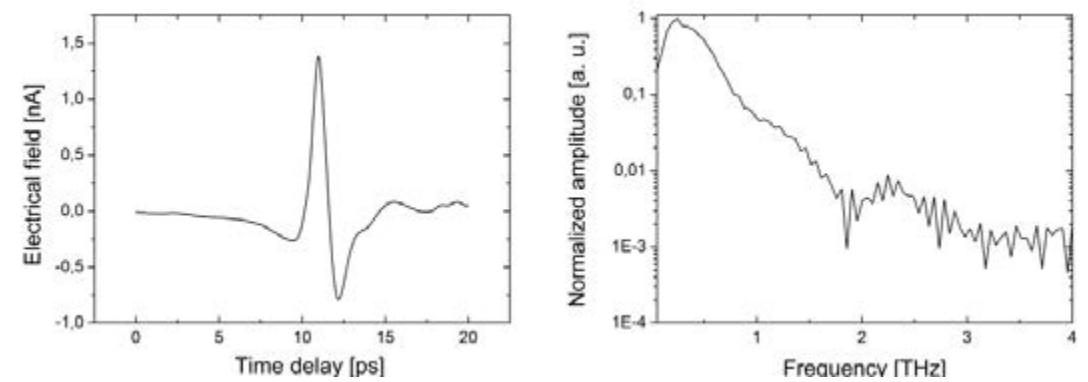

Figure 3.3: THz pulse and its corresponding spectrum measured using the setup shown in Fig. 3.2.

\subsection{Enhancing Spectral Brightness in the Terahertz Band}

Despite of the flexibility provided by THz-TDS systems based on photoconductive antennas the energy of the $\mathrm{THz}$ pulses generated is still low, in the order of fJ [100]. This limits the applicability of this technology in materials with high loss due to the thickness of the piece to be probed or the high attenuation of the material. High spectral brightness is also desirable to increase the dynamic range of 
traditional spectroscopy systems [101] as well as for stand-off applications [102]. Additionally, low radiated power prevents the application of PCAs in nonlinear spectroscopy, which is finding a lot of application in basic physics research [103].

The origin of the low optical-to- $\mathrm{THz}$ conversion efficiency, $10^{-5}$, arises from saturation effects and the limited bias voltage and optical power that antennas can handle [104]. The biasing of small gap sizes of a few $\mu \mathrm{m}$ at tens of $\mathrm{V}$ originates a strong electrical field of a few $\mathrm{kV} / \mathrm{cm}$ across the switch. The breakdown field of the material limits the maximum biasing voltage while the average optical power injected cannot exceed a few tens of $\mathrm{mW}$ because of thermal heating. Therefore, different approaches must be considered to circumvent the problem of low $\mathrm{THz}$ power.

Large area photoconductive antennas (LAPCA) have been proposed to raise emitted $\mathrm{THz}$ power through increased gap areas [105]. High $\mathrm{THz}$ power can be obtained applying bias fields of several $\mathrm{kV}$ to the antenna thanks to increased gap widths in the $\mathrm{cm}$ range. Higher optical powers can be injected due to the enlarged illumination area. Early demonstration of this technique provided $\mathrm{THz}$ pulses with power close to the $\mu \mathrm{J}$ at repetition rates of a few $\mathrm{Hz}[106]$. However, strong material saturation and thermal heating of the device for higher repetition rates are still to be solved. Moreover, the bandwidth of the system is restricted because the $\mathrm{THz}$ energy shifts towards lower frequencies due to reduced acceleration fields and larger electrodes spacing.

Designs based on interdigitated electrodes have been proposed to overcome large thermal loads [107]. Metal electrode width and spacing are of several microns. Odd or even gaps are covered by an additional metallization layer to avoid destructive interference between consecutive gaps due to the opposite sign of the biasing field. The antenna is to be operated using low biasing voltages such as the ones of traditional PCAs while the optical power can be increased according to the enlargement of the illumination area. By using this design an optical-to- $\mathrm{THz}$ conversion ratio of $2 \times 10^{-3}$ has been reported by Beck et al. [108] for optical pulses at a repetition rate of $250 \mathrm{kHz}$ with duration of $50 \mathrm{fs}$ and energy of $4 \mu \mathrm{J}$. The efficiency of this 
approach can be doubled by placing a binary phase mask over the interdigitated structure instead of obscuring every second gap [109]. Thanks to this mask the pulses are slightly delayed in such a way that $\mathrm{THz}$ radiation emitted from the gaps interferes constructively.

To sum up, the combination of interdigitated electrodes along with the enlargement of the illuminated area has been demonstrated to solve the efficiency problems of traditional PCAs.

However, it is not only the $\mathrm{THz}$ source that has a direct influence on the performance of the system. Liu et al. [110] first showed that the use of pulse sequences instead of single pulses circumvents saturation effects and significantly enhances the spectral amplitude of the generated narrowband radiation as compared to broadband generation. Detailed analysis of the physics involved in the process was performed by Weling and Heinz [111]. As opposed to Liu, who generated an ultrafast train of pulses by using spatial phase masks to modulate the optical spectrum, Weling's approach relies on the mixing of a time-dispersed femtosecond pulse with its own delayed replica. This generates a train of $\mathrm{THz}$ pulses whose repetition rate determines the central frequency of the narrowband $\mathrm{THz}$ radiation generated. They named this generation process chirped-pulse mixing (CPM). As shown in Fig. 3.4, for low fluences the effect of CPM is the same as filtering the broadband spectrum. However, for the high-fluence scenario the spectral irradiance does not suffer from saturation in the case of CPM while broadband $\mathrm{THz}$ generation does. The enhancement is due to the reduction of the screening effect which is achieved by spreading the excitation of the material over several intervals or pulses. Thus, saturation of the material occurs at higher fluences compared to single-pulse excitation as long as the pulses are separated long enough for the saturation to decay. Consequently, the more the pulse is stretched the more the saturation fluence is improved. 

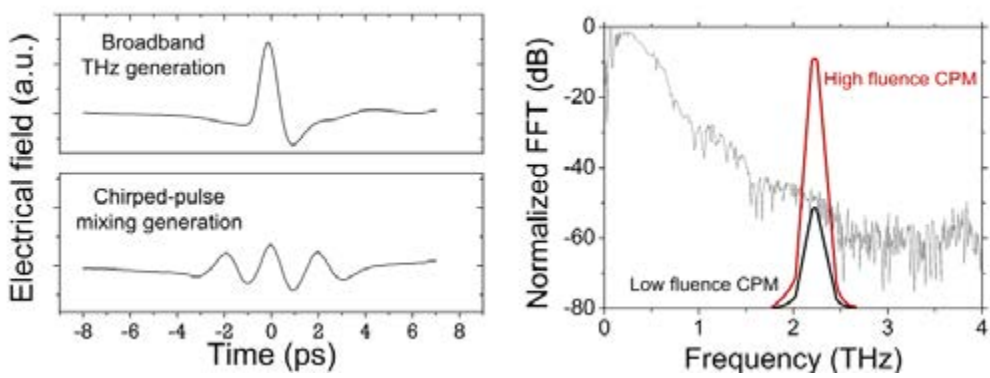

Figure 3.4: Illustration of the mechanism of chirped pulse mixing.

These approaches show that the performance of the system can be locally improved by applying optical processing techniques. Noticeable efforts have been made towards the processing of ultrafast pulses for applications such as phase compensation, pulse compression, selective non-linear spectroscopy, lightwave communications and radiofrequency photonics [112]. The most widely adopted pulse shaping method relies on modulating the spatially dispersed spectral components. A SLM is usually employed to perform both amplitude and phase modulation allowing for computer-controlled reprogrammable pulse shaping. Since the modulation is performed in parallel, signals with a wide bandwidth such as femtosecond pulses can be shaped using slow devices. This scheme has been used to generate narrowband $\mathrm{THz}$ radiation via optical rectification in $\mathrm{ZnTe}$ [113], where radiation spanning the whole range of the crystal was generated for bandwidths as narrow as $140 \mathrm{GHz}$ by means of spectral phase modulation.

Despite good performance of free-space approaches they result in bulky systems susceptible of misalignment. Solutions using fiber technology are compatible with fiber-based THz-TDS systems which circumvent mechanical stability problems. The next sections deal with the implementation of fiber-based solutions able to process femtosecond pulses and generate narrowband $\mathrm{THz}$ radiation. 


\subsubsection{Terahertz Shaping based on Third-Order Dispersion and Self-Phase Modulation}

The dispersive and nonlinear nature of optical fiber has been discussed in Chapter 1. In fiber-based THz-TDS systems, fiber is employed to distribute optical pulses from sources to fiber-pigtailed PCAs. For this scenario dispersion compensation and reduced nonlinear effects are required to reduce pulse distortion as much as possible. However, feeding the antennas with trains of pulses focuses radiation on a given spectral band increasing $\mathrm{THz}$ power density. Shaping of the $\mathrm{THz}$ radiation by controlling nonlinear effects taking place in optical fiber is proposed and demonstrated in Paper D. Breaking of a single optical pulse into a set of pulses through uncompensated third-order dispersion (TOD) enhanced by SPM leads to narrowband $\mathrm{THz}$ generation. EDFAs were used to control the intensity of SPM. Tunability and reconfigurability are possible by changing accumulated dispersion and optical power. The generated radiation was tuned along the whole bandwidth of the THz-TDS system and showed bandwidth reconfigurability. Moreover, the scheme could be implemented with no additional components resulting in a simple and cost-effective solution.

\subsubsection{Terahertz Shaping based on Time-Domain Modulation of the Optical Spectrum}

The processing of ultra-short pulses has traditionally relied on the spatial decomposition of their frequency components to perform spectral modulation. Because traditional modulating devices show a time response much longer than the duration of femtosecond pulses dispersion is required to relax the restrictions. Despite good performance free-space implementations show problems that may be avoided using fiber technology. Spatial dispersion is not simple due to the confined optical field. However, the dispersive character of optical fiber can be employed to perform time-domain spectral decomposition. The spectrum is imprinted in the time domain and can thus be modulated through traditional electro-optical modulation. Pulse shaping [114], frequency conversion [115] and optical delay [116] have been demonstrated using this technique. It allows the generation of large delays in signals of wide bandwidth. A widespread figure of merit 
that accounts not only for the magnitude of the delay but also for the bandwidth of the delayed signal is the time-bandwidth product (TBP).

Time modulation of the spectrum is proposed in paper $\mathbf{E}$. This approach relies on the cross-gain and cross-phase modulation effects (XGM and XPM, respectively) in an interferometric SOA (Semiconductor Optical Amplifier). Pulses are frequency-to-time converted before injection into a Mach-Zehnder interferometric (MZI) structure where they destructively interfere. The system is taken out of destructive interference through the application of an optical signal to one of the arms. Since the same femtosecond source is employed to generate all the shaped pulses the use of electrical generators is not required. Moreover, pulse-by-pulse shaping is possible without any kind of locking. Although approaches based on electrooptical modulation show good performance and flexibility, they are limited by the modulator bandwidth. In our approach the modulation is performed in the optical domain, and is thus restricted by the performance of the SOA. Recovery times below the picoseconds have been demonstrated for SOA structures [117], indicating potential for faster processing than the one obtained using EOMs.

\subsection{Towards Reduced Acquisition Times}

Despite the widespread use of THz-TDS as a fundamental tool in different applications such as material inspection, non-destructive testing, food quality inspection, explosives detection and sensing, the applicability of this technology is restricted to slow time-variant processes due to high acquisition times of THz-TDS systems based on mechanical delay stages. To obtain spectral resolutions in the order of the $\mathrm{GHz}$, the $\mathrm{THz}$ signal recorded must span up to the ns, meaning that the delay stage should move a few tens of $\mathrm{cm}$. Commercial delay stages need at least a few seconds to sweep this range. It is commonly accepted that the main source of noise in THz-TDS systems at low acquisition rates (below the $\mathrm{kHz}$ ) comes from the optical source [118]. The main problem with mechanical delay stages is that they are not able to sweep the whole range in the millisecond range, leading to low dynamic ranges because the relative intensity noise (RIN) of the laser 
is allocated at frequencies below the kilohertz (Fig. 3.5). Thus, averaging becomes essential to perform measurements with acceptable dynamic ranges and acquisition times increase up to several minutes per trace. Moreover, mechanical delay stages are susceptible of calibration errors in both amplitude (imperfect spatial alignment) and delay (calibration errors) and its movement adds noise to the system as long as it is located in the same optical table as the THz-TDS system.

Therefore, to perform fast and high-performance $\mathrm{THz}$ measurements techniques able to implement fast tunable delays that do not rely on mechanical components are necessary. Elzinga et al. [119] proposed a technique to perform pump/probe spectroscopy measurements known as asynchronous optical sampling (ASOPS). It is based on employing two mode-locked lasers with slightly different repetition rates as pump and probe beams. Considering the pump train of pulses as reference, each probe pulse is delayed an increasing duration determined by the difference of the repetition rates. The result is a variation of the optical delay without moving elements. High speed ASOPS has been demonstrated for laser repetition rates of $1 \mathrm{GHz}$ and an offset in the repetition rates of few $\mathrm{kHz}$ [120]. The time required to obtain a $\mathrm{THz}$ trace corresponds to the period of the frequency offset while the delay step can be directly obtained from the repetition rates and the maximum delay corresponds to the smallest of the lasers' periods. Once the THz signal is measured a time conversion is performed from real-time to time-delay as shown in Fig. 3.6. Considering the previous values, $1 \mathrm{~ns}$ is swept in a few tens of $\mu$ s with an approximated step of tens of fs. Due to the high scan rates of these systems the detrimental influence of most of the spectral noise of the optical source, which is usually distributed below $1 \mathrm{kHz}$, is suppressed. Thus, measurements at the shot noise limit can be performed without need for noise suppression devices such as lock-in amplifiers. 


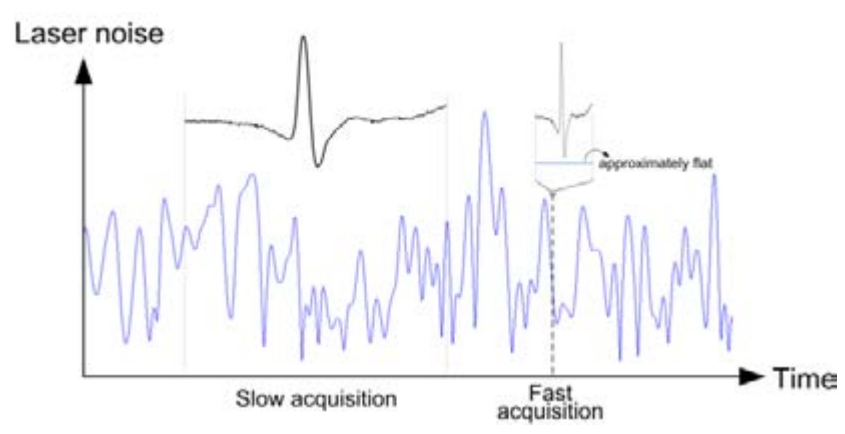

Figure 3.5: Relative intensity noise of the laser in the time domain.
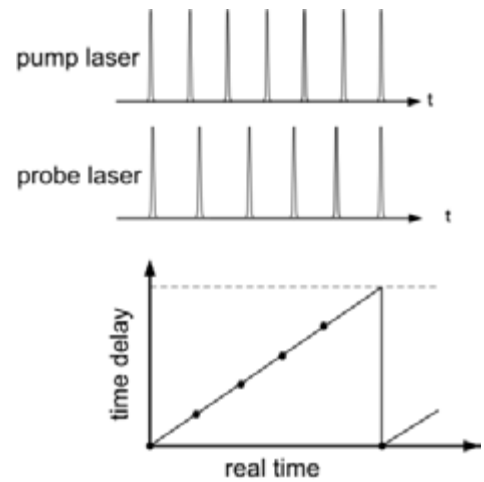

Figure 3.6: Asynchronous optical sampling.

However, for applications requiring shorter scanning ranges most of the swept time is wasted. By increasing the repetition rates it would be possible to reduce the scanning range, but it would be necessary to use optical pulses with lower peak powers for a given maximum power manageable by the PCAs.

A recent approach, known as electronically controlled optical sampling (ECOPS) [121], modifies the scheme of ASOPS by including electronic control to restrict the sweeping range to those parts where meaningful data appear. To do so, both lasers are synchronized in a master and slave configuration by means of a phase-locked loop (PLL), a proportional-integral-differential (PID) actuator and a function generator. While the master laser is free-running the cavity length of the slave laser is controlled by means of a piezoelectric actuator. When the signal generator applies voltage to the phasedetector of the PLL the phase condition of the loop is changed. The 
loop stabilizes the system by applying a voltage to the piezoelectric actuator that changes the cavity length of the slave laser. Therefore, different temporal sweep patterns can be obtained according to the signal applied to the phase detector. In pump/probe experiments such as THz-TDS systems, a square signal is usually the best option since it generates a ramp-type delay.

Despite their good performance, ASOPS and ECOPS require two optical sources. A technique named optical sampling by cavity tuning (OSCAT) which is based on a single ultrafast laser has been recently proposed [122]. It is based on using different lengths for transmitter and receiver paths as well as a single femtosecond fiber laser with a piezoelectric actuator attached to its cavity to slightly change the repetition frequency. According to differences in distribution paths a different repetition rate is applied to the PCAs at the same time. Its work of principle is very much a simplification of ECOPS but saving one of the optical sources by means of temporal multiplexing.

Techniques previously reported rely on the modification of the optical source. Processing of pulses already generated is another possibility which does not rely on the modification of the source.

Different media can be employed to implement optical delays [123] although we focus on fiber-based solutions. In this context, the TBP relates implemented delays and bandwidth of the delayed signal. Large TBP are in general desirable because they allow large delays for wideband signals. Most solutions based on non-linear effects such as SBS [124] or SRS [125] provide small TBP due to their limited bandwidth. The SPM effect was recently reported to implement large delays despite severe pulse widening [126], which is not useful for spectroscopy applications but could be employed in communications applications. Solutions based on gain saturation in a SOA are interesting because even when small delays are obtained, the solution is wideband and highly scalable [127]. Schemes based on electrooptical modulation of the optical spectrum have also been reported through phase [128] and amplitude modulation [116]. Chirped-fiber Bragg gratings (CFBG) or optical fiber are employed to perform frequencyto-time and time-to-frequency conversion, as in paper $\mathbf{E}$. When dealing with femtosecond pulses optical fiber is the best choice due to 
the limited bandwidth of CFBGs, although residual TOD limits the performance of the system.

Next sections focus on the study of alternative schemes able to delay femtosecond pulses. Special emphasis is made on techniques able to sweep long delay ranges at repetition rates over the bandwidth of the optical source RIN, thus the LIA could be removed.

\subsubsection{Pulse Delay based on Gain Saturation in Semiconductor Optical Amplifiers}

When an intense optical field is injected into an SOA its gain and phase profiles temporally change due to carrier density variations arising from saturation. These changes apply to the saturating signal and any other signal injected. This mechanism was reported in paper E to generate a pulse shaper. By using chirped pulses, instead of transform-limited ones, the different spectral components will suffer from different amplitude and phase modulation. Paper $\mathbf{F}$ reports how this modulation can be employed to delay, or advance, femtosecond pulses according to the sign of the chirped pulses. The magnitude of the time shift directly depends on the electrical current biasing the SOA since it is related to its recovery time. Little to no degradation was observed on the performance of the pulses for the whole delay range. Thus, the technique is suitable for THz-TDS systems as reported in the paper since no changes were appreciated in the $\mathrm{THz}$ spectrum for different delays. Although delays obtained are not large enough to sweep $\mathrm{THz}$ traces, the lack of pulse degradation suggests it could be done by cascading several SOAs and using pulses with stronger chirp. The bandwidth of the electrical port of the SOA, already reported in [129], is large enough to perform measurements at $\mathrm{MHz}$ rates.

\subsubsection{Optical Delay Line based on Frequency-to- Time Modulation}

Electrooptical modulation of the optical spectrum of a pulsed source seems to be an interesting approach because it is not fundamentally limited in bandwidth, meaning that in principle ultra-short pulses could be delayed without severe degradation. The main concept 
behind delay through time modulation of spectra is domain switching. As discussed in Chapter 1, the modulation of a CW laser with a LO microwave tone generates different frequency terms (sidebands around the optical carrier). By chirping ultra-short pulses the frequency spectra is being brought to the time domain. Analogously, the time modulation of such chirped signals originates additional terms in the time domain. Delays implemented using this technique depend on both chirp and modulation frequency. Thus, tunable optical delay lines may be obtained employing tunable electrical sources. The main problem of this approach is that several pulses are generated and additional filtering stages are necessary to select the optical pulse. In paper $\mathbf{G}$ the use of a complex modulation such as single-sideband suppressed-carrier (SSB-SC) is proposed to achieve single pulse generation. This approach does not need additional stages and provides tuning ranges large enough to sample $\mathrm{THz}$ traces. Although pulses are widened up to the picosecond range due to TOD of the optical fiber employed to perform frequency-to-time conversion, techniques that have been proposed to compensate for the TOD of fiber links [130] should be considered to provide transform-limited pulses. The tuning speed of available local oscillator allows sweeping of the whole delay range at high frequencies, potentially reducing the effect of RIN noise on recorded $\mathrm{THz}$ traces. 


\section{Chapter 4}

\section{General discussion}

The previous chapters provided the necessary background to understand the ideas developed in the appended papers as well as a brief review of the main ideas developed. The context and motivation behind each idea have also been addressed. This chapter is dedicated to discuss the results obtained.

For photonic microwave filters the work was oriented towards the development of techniques providing enhanced performance compared with pure electrical solutions. The first idea provides dynamic reconfigurability of the response of transversal photonic filters based on dispersion and multiwavelength sources as well as a reduction in the number of components. The measurements performed showed an increase in the number of taps thanks to cascaded effect of degenerative four-wave mixing controlled by Er-doped fiber amplification. The bandpass frequency is tuned independently from the filter shape by changing the emission wavelength of the optical source thanks to the large bandwidth of FWM. Additional control on the coefficients' amplitude distribution was provided by the optical filter implemented through the combined effect of a piece of PMF and the polarization dependence of our $\mathrm{LiNbO}_{3}$-based Mach-Zehnder 
modulator. This allowed the implementation of particular filter shapes, such as a uniform response and a Hanning window. To sum up, we propose a filter whose response can be dynamically controlled and is independent of the bandpass frequency, which is easily tuned and only limited by the bandwidth of the photodetector.

The next two papers focused on the design of single sideband filters compatible with RF applications. Both are based on direct sampling of narrow optical filters in the electrical domain. The first approach employs a phase-shifted fiber Bragg grating. The phase shift could be induced by means of different elements, although the experimental measurements were performed using a piezoelectric actuator. A Mach-Zehnder modulator was single-sideband modulated and its emission wavelength placed next to the grating response in such a way that frequencies modulating the optical carrier experience attenuation except for the transmission peak induced by the phase shift. The demonstrated bandwidth could be further decreased by employing smaller stretching devices, while the tuning range of the filter could be extended by using gratings with a wide response such as chirped FBGs.

The last paper of Chapter 2 also relied on the sampling of optical responses. In this case a microring resonator fabricated in silicon was employed and the optical carrier was phase-modulated. Thus, single bandpass responses are obtained from notch filters as opposed to the conventional need of an optical bandpass filter to implement a bandpass microwave response using single-sideband modulation of the optical carrier. Although experimental results were not able to sample the whole response due to the limited bandwidth of the phasemodulator available, the approach should provide responses much narrower that the one reported in the paper by design of advanced resonators. Continuous development of photonic integration technologies is expected to provide compact and low-cost integrated filters such as the one proposed, since this architecture employs a simple EOM and all the components allow for monolithic integration on a single photonic chip.

Chapter 3 was dedicated to the study of fiber-based $\mathrm{THz}$ processing techniques. Free-space components have been traditionally 
employed to build $\mathrm{THz}$ setups. However, recent availability of photoconducting materials compatible with $1.5 \mu \mathrm{m}$ light allows the application of fiber-based processing techniques to these systems, providing advantages in terms of size and stability. This chapter addressed two main problems of THz-TDS systems: limited power and long acquisition times. Pulse shaping can solve the former by focusing the energy into narrow emission bands. Two schemes have been proposed to solve this problem. The first one relies on nonlinear effects taking place in the optical fiber used to distribute pulses. The amount of optical power controls the intensity of the SPM effect, which is used to break the original pulse into a set of subpulses originated from TOD. Accordingly, the central frequency of the radiation depends on the amount of TOD while the intensity of SPM affects the bandwidth of the radiation. The experimental results presented agreed well with performed numerical simulations and the FWHM emission bandwidth was considerably reduced. This architecture shows potential for simple focusing of the radiation without requiring additional elements. The second architecture is based on modulating the optical spectrum in the time domain. The dispersion of the standard fiber employed to distribute the pulses performs frequency-to-time conversion and the spectral components are differently modulated in an interferometric SOA structure using shaping optical pulses to change the interference condition. Experimental results show independent tunability and reconfigurability of the $\mathrm{THz}$ radiation for the whole bandwidth of the THz-TDS system. The proposed scheme is based on dispersion, which is inherent to the propagation of pulses through optical fiber, and the same optical source that is employed to generate the pulses is also employed as the shaping signal. Thus, the system shows potential to be implemented without requiring more elements than the SOA interferometer.

One of the main limiting factors when performing measurements of $\mathrm{THz}$ traces is the acquisition time. Due to the limited movement speed of mechanical elements the relative intensity noise of the optical source considerably reduces the dynamic range of the system and averaging becomes necessary, further increasing the measurement time. To solve this problem we propose the implementation of delay 
lines using different techniques. One of them is based on the gain saturation of an SOA by chirped pulses. Thus, the spectral components which enter first on the SOA observe different gain and phase shift than the rest of frequencies. When recompressing the pulse a different delay or advance is obtained according to the sign of the initial dispersion. The problem with this technique is that delays obtained are not that large, in the range of a few pulse widths. However, due to small pulse degradation the system is highly scalable and cascaded SOAs should provide increased delays and advances.

The last paper deals with the implementation of larger delays through frequency-to-time conversion, similarly to the previously commented pulse shaping approach. Single-sideband suppressed-carrier modulation allows for the generation of single pulses as opposed to previously reported solutions which require further processing of the generated waveforms. Advances and delays large enough to provide $\mathrm{GHz}$ resolution in the measured spectra were generated, although pulse widening is a problem that must be addressed. However, phase modulation of the stretched pulses has already been demonstrated to reduce pulse widening and could be combined with the proposed scheme to provide such large delays without high degradation. It must be highlighted how all of the processing techniques for terahertz systems proposed along the thesis are based on basic properties of optical fiber: dispersion and nonlinear effects. Detrimental effects of the propagation medium become useful for processing applications. Moreover, fast SOAs and signal generators able to sweep $\mathrm{THz}$ traces at frequencies higher than the noise content of the optical source are available, meaning that the proposed solutions do not only implement optical delays without moving elements but are also able to perform faster measurements with increased dynamic range. 


\section{Chapter 5}

\section{Conclusion}

Optical fiber has become the preferred transmission medium for data exchange due to its excellent properties. Its low losses, especially at $1.5 \mu \mathrm{m}$, allow for long distance transmission of vast amounts of data. Additionally it is robust, flexible, cheap, and has low-weight and volume. These features motivate research and development efforts to satisfy the demands of the information society which is based on optical data transmission. The resulting technology can also be exploited to benefit other regions of the electromagnetic spectrum. A signal in another band can be converted to a new space where it can be processed, transmitted and then converted back to its original frequency. Although at first more complex than direct processing, this conversion opens new possibilities and allows functionalities and performance not attainable in the original domain. In practice the optical processing of signals is only limited by the bandwidth of electrooptic and optoelectronic converters.

This approach has been followed in the Thesis to obtain new functionalities in the microwave and $\mathrm{THz}$ regions of the spectrum. The applications in these two bands followed different paths. While microwave photonics developed parallel to the telecommunication 
industry aiming to provide increased performance to data processing and distribution, the $\mathrm{THz}$ range has long been dedicated to basic sciences. However, the recent availability of photoconductive materials which can be fed using telecom light not only shows potential to reduce the cost of the system but also allows for fiber-based processing techniques. Thus, the bandwidth of electrical signals that can be processed using these techniques comprises now more than 6 decades, from a few $\mathrm{MHz}$ to several $\mathrm{THz}$.

In this Thesis different approaches performing optical processing in both the microwave and terahertz band were addressed. Photonic filters with comparably increased performance with respect to pure electrical solutions were studied. Although the experimental results were obtained at microwave frequencies the principle of operation of the proposed techniques is still valid at $\mathrm{THz}$ frequencies if suitable electrooptic and optoelectronic conversions are available. The study focused on obtaining dynamically reconfigurable and single-bandpass responses. The contributions made are significant because they reduce the cost of photonic microwave finite impulse response filters based on multiwavelength sources and dispersive medium, and propose novel techniques to obtain single-bandpass responses based on simple optical filters with applicability to RF systems and potential for monolithic integration. The processing of ultra-short pulses is also addressed by using fiber-based elements. In this Thesis, dispersive and nonlinear effects taking place in optical fiber are employed to perform optical processing in a simple way while circumventing inconvenient mechanical technologies. Techniques with potential to increase the performance of current $\mathrm{THz}$ systems are investigated. Original concepts for shaping of $\mathrm{THz}$ radiation to obtain increased spectral brightness through concentration of the $\mathrm{THz}$ energy in narrow bandwidths are reported, as well as the initial development of optical delay lines with no moving parts but potential of greatly enhancing the acquisition speed of THz-TDS systems. Thus, the two main limitations problems of current $\mathrm{THz}$ technology, low power and long acquisition times, are addressed.

To sum up, the excellent performance of optical fiber as transmission medium enables not only long distance data transmission 
but also processing of a wide range of frequencies in the optical domain. Optical processing is appealing because it enables functionalities not attainable by processing in other wavelengths. In a practical scenario the maximum frequency that can be processed is given by the conversion bandwidth. In this Thesis several fiber-based processing techniques are studied. Although they are demonstrated at certain frequency ranges all of them are applicable to any other band supported by the optical fiber. 



\section{References}

[1] A. Kersey, M. Davis, H. Patrick, M. LeBlanc, K. Koo, C. Askins, M. Putnam, and E. Friebele, "Fiber grating sensors," J. Lightwave Technol., vol. 15, no. 8, pp. $1442-1463$, Aug. 1997.

[2] A. Katzir, Lasers and Optical Fibers in Medicine. Physical Techniques in Biology and Medicine, (Academic Press, San Diego, 1993).

[3] A. J. Seeds, “Microwave photonics," IEEE Trans. Microw. Theory Tech., vol. 50, no. 3, pp. 877-887, Mar. 2002.

[4] A. E. Siegman, Lasers. Sausalito, CA: University Science Books, 1986.

[5] T. H. Maiman, "Stimulated optical radiation in ruby masers," Nature, vol. 187, pp. 493-494, 1960.

[6] I. Hayashi, M. B. Panish, P. W. Foy, and S. Sumski, "Junction lasers which operate continuously at room temperature," Appl. Phys. Lett., vol. 17, no. 3, pp. 109-111, Aug. 1970.

[7] R. J. Mears, L. Reekie, I. M. Jauncey, and D. N. Payne, "Low-noise Erbium-doped fibre amplifier operating at $1.54 \mu \mathrm{m}$," Electron. Lett., vol. 23, no. 19, pp. 1026-1028, Sept. 1987.

[8] H. Lamm, "Biegsame optische Geräte," Z. Instrumentenk, vol. 50, pp. 579-581, 1930.

[9] A. C. S. van Heel, "A new method of transporting optical images without aberrations," Nature, vol. 173, p. 39, 1954.

[10] T. Miya , Y. Terunuma, T. Hosaka, and T. Miyoshita, "Ultimate lowloss singlemode fibre at $1.55 \mu \mathrm{m}$," Electron. Lett., vol. 15, pp. 106-108, Feb. 1979.

[11] G. P. Agrawal, Fiber-Optic Communication Systems, 2nd ed. (Wiley, New York, 1997).

[12] G. P. Agrawal, Nonlinear fiber optics, 3rd ed. (Academic Press, 2001). 
[13] H. Sunnerud, M. Karlsson, C. Xie, and P. A. Andrekson, "Polarizationmode dispersion in high-speed fiber-optic transmission systems," $J$. Lightwave Technol., vol. 20, pp. 2204-2219, Dec. 2002.

[14] J. Noda, K. Okamoto, and Y. Sasaki, "Polarization-maintaining fibers and their applications," J. Lightwave Technol., vol. 4, no. 8, pp. 10711089, Aug. 1986.

[15] V. Ramaswamy, W. G. French, and R. D. Standley, "Polarization characteristics of noncircular core single-mode fibers," Appl. Opt., vol. 17, pp. 3014-3017, 1978.

[16] H. Ito, T. Furuta, S. Kodama, and T. Ishibashi, "InP/InGaAs unitravelling-carrier photodiode with $310 \mathrm{GHz}$ bandwidth," Electron. Lett., vol. 36 , no. 21 , pp. 1809-1810, Oct. 2000.

[17] H. Ito, T. Furuta, F. Nakajima, K. Yoshino, and T. Ishibashi, "Continuous THz-wave generation using uni-travelling-carrier photodiode," 15th International Symposium on Space Terahertz Technology, pp. 143-150, Massachusetts, April 27-29 2004.

[18] J. Capmany and D. Novak, "Microwave photonics combines two worlds," Nat. Photon., vol. 1, no. 6, pp. 319-330, Jun. 2007.

[19] K. Wilner, A.P. Van Den Heuvel, "Fiber Optic Delay Lines for Microwave Signal Processing," Proceedings of the IEEE, vol. 64, pp. 805$807,1976$.

[20] K.P. Jackson, H.P. Shaw, "Fiber-optic delay-line signal processors", Chapter 7.1, Optical Signal Processing, J.L. Horner editor, pp. 431-476, 1987.

[21] S. Tedjini, A. Ho-Quoc, D.M. Khalil, "All-optical networks as microwave and millimetre-wave circuits", IEEE Transactions on Microwave Theory and Techniques, vol. 43, no. 9, pp. 2428-2434, September 1995.

[22] H. Zmuda, E.N. Toughlian, "Photonic Aspects of Modern Radar", Ed. Artech House, London, 1994.

[23] B. M. Hendrickson, G.A. Koepf, "Optoelectronic Signal Processing for Phased-Array Antennas II," Proceedings of the SPIE (The International Society for Optical Engineering), vol. 1217, 16-17 January, 1990, Los Angeles.

[24] B. M. Hendrickson, G.A. Koepf, "Optoelectronic Signal Processing for Phased-Array Antennas IV," Proceedings of the SPIE (The International Society for Optical Engineering), vol. 2155, 26-27 January, 1994, Los Angeles. 
[25] N. A. Riza, "Selected Papers on Photonic Control Systems for Phased Array Antennas," SPIE Milestone Series, Volume MS- 136, 1997.

[26] B. Jalali, "Time-stretch and wavelength-division sampling: photonic preprocessing techniques for ultrafast analog-to-digital conversion," IEEE LEOS Summer Topical Meeting, pp. 194-1995, Vancouver (Canada), July 2003.

[27] J. Chou, Y. Han, B. Jalali, "Adaptive RF-Photonic Arbitrary Waveform Generator," IEEE Photonics Letters, vol. 15, no. 4, pp. 581-583, April 2003.

[28] B. Vidal, V. Polo, J. L. Corral, J. Martí, "Multibeam Optical Beamforming Architectures for Broadband Wireless Access Networks," IST Mobile \& Wireless Telecommunications Summit 2003, Aveiro (Portugal), 15-18 June, 2003.

[29] J. Capmany, D. Pastor, A. Martinez, B. Ortega, S. Sales, "Microwave photonic filters with negative coefficients based on phase inversion in an electro-optic modulator," Optics Letters, vol. 28, no. 16, pp. 1415-1417, 15th August 2003.

[30] A. Oppenheim and R. Schaffer, "Discrete Time Signal Processing," Englewood Cliffs, NJ, Prentice Hall, 1989.

[31] R.E. Collin, "Foundations of microwave engineering," Ed. International, 2nd ed., New York 2001.

[32] J. Capmany, B. Ortega, and D. Pastor, "A Tutorial on Microwave Photonic Filters," J. Lightwave Technol., vol. 24, no. 1, pp. 201-229, Jan. 2006.

[33] J. Capmany, D. Pastor, and B. Ortega, "New and flexible fiber-optic delay-line filters using chirped fiber Bragg gratings and laser arrays," IEEE Transactions on Microwave Theory and Techniques, vol. 47, no. 7, pp. 1321-1326, July 1999.

[34] M. Born, E. Wolf, "Principles of Optics," Ed. Pergamon Press, 6th ed., New York, Chapter 10, 1980.

[35] C.T. Chang, J.A. Cassaboom, H.F. Taylor, "Fiber optic delay line devices for RF signal processing," Electronics Letters, vol. 13, pp. 678$680,1977$.

[36] Y.-S. Lee, "Principles of Terahertz Science and Technology," Springer, New York, 2009. 
[37] J.Waldman, H. R. Fetterman, P. E. Duffy, T.G. Bryant, and P. E. Tannenwald, "Submillimeter model measurements and their applications to millimeter radar systems," in Proc. 4th Int. Infrared NearMillimeterWaves Conf., Dec. 1979, pp. 49-50.

[38] A. Bergner, U. Heugen, E. Brundermann, G. Schwaab, M. Havenith, D. R. Chamberlin, and E. E. Haller, "New p-Ge THz laser spectrometer for the study of solutions: $\mathrm{THz}$ absorption spectroscopy of water," Rev. Sci. Instrum., vol. 76, no. 6, 063110, Juny 2005.

[39] G. L. Carr, M. C. Martin, W. R. McKinney, K. Jordan, G. R. Neil, and G. P. Williams, "High-power terahertz radiation from relativistic electrons," Nature, vol. 420, pp. 153-156, Nov. 2002.

[40] C. A. Brau, "Free-electron lasers", Science, vol. 239, p. 4844, March 1988.

[41] G. Kozlov and A. Volkov, "Millimeter and Submillimeter Wave Spectroscopy of Solids", edited by G. Grüner (Springer-Verlag, Berlin, 1998), Chap. 3.

[42] A. Maestrini, J. S. Ward, J. J. Gill, H. S. Javadi, E. Schlecht, C. TriponCanseliet, G. Chattopadhyay, and I. Mehdi, "A 540-640-GHz HighEfficiency Four-Anode Frequency Tripler", IEEE Trans. Microw. Theory Tech., vol. 53, no. 9, pp. 2835-2843, Sept. 2005.

[43] Jerome Faist, Federico Capasso, Deborah L. Sivco, Carlo Sirtori, Albert L. Hutchinson, and Alfred Y. Cho, "Quantum Cascade Laser", Science, vol. 264, pp. 553-556, April 1994.

[44] K. H. Yang, P. L. Richards, and Y. R. Shen, "Generation of Far-Infrared Radiation by Picosecond Light Pulses in LiNb03," Appl. Phys. Lett., vol. 19, no. 1, pp. 320-323, Nov. 1971.

[45] F. Zernike and P. R. Berman, "Generation of far infrared as a difference frequency," Phys. Rev. Lett., vol. 15, no. 26, p. 999, 1965.

[46] D. H. Auston, "Picosecond optoelectronic switching and gating in silicon," Appl. Phys. Lett., vol. 26, no. 3, pp. 101-103, 1975.

[47] Q. Wu and X.-C. Zhang, "Free-space electro-optics sampling of midinfrared pulses," Appl. Phys. Lett., vol. 71, no. 10, p. 1285-1286, Sept. 1997.

[48] P. R. Smith, D. H. Auston and M. C. Nuss, "Subpicosecond photoconducting dipole antennas," IEEE Journal of Quantum Electronics, vol. 24, no. 2, p. 255-260, Feb. 1988. 
[49] M. van Exter, Ch. Fattinger, and D. Grischkowsky, "Terahertz timedomain spectroscopy of water vapor," Opt. Lett., vol. 14, no. 20, pp.1128-1130, Oct. 1989.

[50] B. Sartorius, D. Stanze, T. Göbel, D. Schmidt, and M. Schell, "Continuous wave terahertz systems based on $1.5 \mu \mathrm{m}$ telecom technologies ," J Infrared Milli Terahz Waves, Vol. 33, No. 4, pp. 405417, April 2012.

[51] M. J. E. Golay, "Theoretical and practical sensitivity of the pneumatic infrared detector," Rev. Sci. Instr., vol. 20, pp. 816-820, 1949.

[52] P. L. Richards, "Bolometers for infrared and millimeter waves," J. Appl. Phys., vol. 76, no. 1, pp. 1-24, 1994.

[53] H. P. Beerman, "The pyroelectric detector of infrared radiation," IEEE Trans. on Electron Devices, vol. 16, no.6, pp. 554-557, Jun 1969.

[54] M. J. F. Digonnet, "Rare-earth-doped fiber lasers and amplifiers," (CRC Press, NY, 2001), Chap. 8.

[55] M. Nagai, K. Tanaka, H. Ohtake, T. Bessho, T. Sugiura, T. Hirosumi, andM. Yoshida, "Generation and detection of terahertz radiation by electro-optical process in GaAs using $1.56 \mu \mathrm{m}$ fiber laser pulses," Appl. Phys. Lett., vol. 85, pp. 3974-3976, 2004.

[56] F. Pan, G. Knopfle, Ch. Bosshard, S. Follonier, R. Spreiter, M. S. Wong, and P. Gunter, "Electrooptic properties of the organic salt 4-N,Ndimethylamino-4'-N'-methyl-stilbazolium tosylate," Appl. Phys. Lett., vol. 96, pp. 13-15, 1996.

[57] Ch. Bosshard, K. Sutter, Ph. Pretre, J. Hulliger, M. Florsheimer, P. Kaatz, and P. Guunter, "Organic Nonlinear Optical Materials," Advances in Nonlinear Optics Vol. 1, Gordon and Breach, Amsterdam, 1995.

[58] A. Takazato, M. Kamakura, T. Matsui, J. Kitagawa, and Y. Kadoya, "Detection of terahertz waves using low-temperature-grown InGaAs with 1.56 $\mathrm{mm}$ pulse excitation," Appl. Phys. Lett., vol. 90, no. 10, 101119, 2007.

[59] F. Ospald, D. Maryenko, K. von Klitzing, D. C. Driscoll, M. P. Hanson, H. Lu, A. C. Gossard, and J. H. Smet, "1.55 $\mu \mathrm{m}$ ultrafast photoconductive switches based on ErAs:InGaAs," Appl. Phys. Lett., vol. 92, 131117, 2008.

[60] N. Chimot, J. Mangeney, L. Joulaud, P. Crozat, H. Bernas, K. Blary, and J. F. Lampin, "Terahertz radiation from heavy-ion-irradiated 


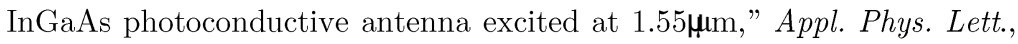
vol. 87 , no. 19, 193510, 2005.

[61] A. Fekecs, M. Bernier, D. Morris, M. Chicoine, F. Schiettekatte, P. Charette, and R. Arès, "Fabrication of high resistivity cold-implanted InGaAsP photoconductors for efficient pulsed terahertz devices," Opt. Mater. Express, vol. 1, no. 7, pp. 1165-1177, 2011.

[62] R. J. B. Dietz, M. Gerhard, D. Stanze, M. Koch, B. Sartorius, and M. Schell, "THz generation at $1.55 \mu \mathrm{m}$ excitation: six-fold increase in $\mathrm{THz}$ conversion efficiency by separated photoconductive and trapping regions," Opt. Express, vol. 19, no. 27, pp.25911-25917, 2011.

[63] H. Erlig, S. Wang, T. Azfar, A. Udupa, H. R. Fetterman, and D. C. Streit, "LT-GaAs detector with 451 fs response at $1.55-\mu \mathrm{m}$ via twophoton absorption," Electron. Lett., vol. 35, no. 2, pp. 173-174, 1999.

[64] G. Klatt, B. Surrer, D. Stephan, O. Schubert, M. Fischer, J. Faist, A. Leitenstorfer, R. Huber, and T. Dekorsy, "Photo-Dember terahertz emitter excited with an Er:fiber laser," Appl. Phys. Lett., vol. 98, 021114, 2011.

[65] J. R. Middendorf and E. R. Brown, "THz generation using extrinsic photoconductivity at $1550 \mathrm{~nm}, "$ Opt. Express, vol. 20, pp. 16504-16509, 2012.

[66] C. H. Lee, "Picosecond optoelectronic switching in GaAs," Appl. Phys. Lett., vol. 30, no. 2, pp. 84-86, 1977.

[67] P. R. Smith, D. H. Auston, and W. M. Augustyniak, "Measurement of GaAs field-effect transistor electronic impulse response by picosecond optical electronics," Appl. Phys. Lett., vol. 39, no. 9, pp. 739-741, 1981.

[68] G. Mourou, C. V. Stancampiano, A. Antonetti, and A. Orszag, "Picosecond microwave pulses generated with a subpicosecond laser-driven semiconductor switch," Appl. Phys. Lett. vol. 39, no. 4, pp. 295-296, 1981.

[69] D. H. Auston and P. R. Smith, "Generation and detection of millimeter waves by picosecond photoconductivity," Appl. Phys. Lett., vol. 43, no. 7, pp. 631-633, 1983.

[70] P. R. Smith, D. H. Auston and M. C. Nuss, "Subpicosecond photoconducting dipole antennas," IEEE J. Quantum Electron., vol. 24, no. 2, pp. 255-260, Feb. 1988.

[71] S. Gupta, M. Frankel, J. Valdmanis, J. Whitaker, G. Mourou, F. Smith, and A. Calawa, "Subpicosecond carrier lifetime in GaAs grown by 
molecular beam epitaxy at low temperatures," Appl. Phys. Lett. vol. 59, no. 25, pp. 3276-3278, 1991.

[72] S. L. Dexheimer, Terahertz Spectroscopy: Principles and Applications. Rochester-New York: CRC Press, 2008.

[73] J. Capmany, B. Ortega, D. Pastor, and S. Sales, "Discrete-time optical processing of microwave signals," J. Lightwave Technol., vol. 23, no. 2, pp. 702-723, 2005.

[74] J. Capmany, D. Pastor, and B. Ortega, "New and flexible fiber-optic delay-line filters using chirped fiber Bragg gratings and laser arrays," IEEE Trans. Microw. Theory Tech., vol. 47, no. 7, pp. 1321-1326, 1999.

[75] A. P. Foord, P. A. Davies, and P. A. Greenhalgh, "Synthesis of microwave and millimetre-wave filters using optical spectrum-slicing," Electron. Lett., vol. 32, no. 4, pp. 390-391, Feb. 1996.

[76] V. R. Supradeepa, C. M. Long, R. Wu, F. Ferdous, E. Hamidi, D. E. Leaird, and A. M. Weiner, "Comb-based radiofrequency photonic filters with rapid tunability and high selectivity," Nat. Photon., vol. 6, no. 3, pp. 186-194, Feb 2012.

[77] B. Vidal, V. Polo, J. L. Corral, and J. Martí, "Efficient architecture for WDM photonic microwave filters," IEEE Photon. Technol. Lett., vol. 16, no. 1, pp. 257-259, January 2004.

[78] R. A. Minasian, K. E. Alameh, and E. H. W. Chan, "Photonics-based interference mitigation filters," IEEE Trans. Microw. Theory Tech., vol. 49, no. 10, pp. 1894-1899, Oct 2001.

[79] B. Vidal, V. Polo, J. L. Corral, and J. Martí, "Harmonic suppressed photonic microwave filter," J. Lightwave Technol., vol. 21, no. 12, pp. 3150-3154, Dec 2003.

[80] J. Mora, B. Ortega, A. Díez, J. L. Cruz, M. V. Andrés, J. Capmany, and D. Pastor, "Photonic microwave tunable single-bandpass filter based on a Mach-Zehnder interferometer," J. Lightwave Technol., vol. 24, no. 7, p. $2500,2006$.

[81] J. Mora, L.R. Chen, and J. Capmany, "Single-bandpass microwave photonic filter with tuning and reconfiguration capabilities," $J$. Lightwave Technol., vol. 26, no. 15, pp. 2663-2670, 2008.

[82] T. X. H. Huang, X. Yi, and R. A. Minasian, "Single passband microwave photonic filter using continuous-time impulse response," Opt. Express, vol. 19, no. 7, pp. 6231, Mar 2011. 
[83] S. Xiao and A. M. Weiner, "Coherent photonic processing of microwave signals using spatial light modulators: Programmable amplitude filters," J. Lightw. Technol, vol. 24, no. 7, pp. 2523-2529, Jul 2006.

[84] B. Vidal, M. A. Piqueras, and J. Martí, "Tunable and reconfigurable photonic microwave filter based on stimulated Brillouin scattering," Opt. Lett., vol. 32, no. 1, pp. 23-25, Jan 2007.

[85] R. W. Boyd, Nonlinear Optics, Third Edition, 3rd ed. Academic Press, Apr 2008.

[86] C.J. McKinstrie and M.G. Raymer, "Four-wave mixing cascades near the zero-dispersion frequency," Opt. Express, vol. 14, pp. 9600, 2006.

[87] A. Cerqueira, J.M. Chavez Boggio, A.A. Rieznik, H.E. HernándezFigueroa, H.L. Fragnito, and J.C. Knight, "Highly efficient generation of broadband cascaded four-wave mixing products", Opt. Express, vol. 16, pp. 2816, 2008.

[88] A. Othonos and K. Kalli, Fiber Bragg Gratings: Fundamentals and Applications in Telecommunications and Sensing. Boston-London: Artech House, 1999.

[89] G. P. Agrawal and S. Radic, "Phase-shifted fiber Bragg gratings and their application for wavelength demultiplexing," IEEE Photon. Technol. Lett., vol. 6, no. 8, pp. 995-997, Aug 1994.

[90] L. Li, A. Schlzgen, X. Zhu, J. V. Moloney, J. Albert, and N. Peyghambarian, "1 w tunable dual-wavelength emission from cascaded distributed feedback fiber lasers," Appl. Phys. Lett., vol. 92, Mar 2008, paper 051111.

[91] R. J.Williams, N. Jovanovic, G. D. Marshall, and M. J.Withford, "Optically switched erbium fibre laser using a tunable fibre-Bragg grating," in Proc. SPIE, vol. 7580, pp. 75800P-1-75800P1-8.

[92] P. Pérez-Millán, J. L. Cruz, and M. V. Andrés, "Active Q-switched distributed feedback erbium-doped fiber lasers," Appl. Phys. Lett., vol. 87 , no. 1, 011104, 2005 .

[93] G. E. Villanueva, P. Pérez-Millán, J. Palací, J. L. Cruz, M. V. Andrés, and J. Martí, "Dual-wavelength DFB erbium-doped fiber laser with tunable wavelength spacing," IEEE Photon. Technol. Lett., vol. 22, no. 4, pp. 254-256, Feb 2010.

[94] J. Heebner, R. Grover and T. A. Ibrahim, "Optical microresonators. Theory, fabrication and applications," (Springer Series in Optical Sciences, USA, 2008). 
[95] S. A. Crooker, "Fiber-coupled antennas for ultrafast coherent terahertz spectroscopy in low temperatures and high magnetic fields," Rev. of Sci. Instrum., vol. 73, no. 9, pp. 3258-3265, Sep 2002.

[96] B. Sartorius, H. Roehle, H. Künzel, J. Böttcher, M. Schlak, D. Stanze,H. Venghaus, and M. Schell, "All-fiber terahertz time-domain spectrometer operating at $1.5 \mu \mathrm{m}$ telecom wavelengths", Opt. Express, vol. 16, no. 13, pp. 9565-9570, Jun 2008.

[97] http://www.menlosystems.com/media/pdf/DataSheet/TERA15FC DataSheet.pdf

[98] J. Palací and B. Vidal, "Distributed THz Transmitter/Receiver based on a 1.5 $\mathrm{m}$ Fiber Link," in 36th International Conference on Infrared, Millimeter and Terahertz Waves. IRMMW-THz 2011, Houston, Texas, Oct 2011 .

[99] F. Eichhorn, R. K. Olsson, J. C. D. Buron, L. Grüner-Nielsen, J. E. Pedersen, and P. U. Jepsen, "Optical fiber link for transmission of 1-nJ femtosecond laser pulses at $1550 \mathrm{~nm}$," Opt. Express, vol. 18, no. 7, pp. 6978-6987, 2010.

[100] Y. Huang, N. Khiabani, and S. Yaochun, "Terahertz photoconductive antenna efficiency," 2011 international Workshop on Antenna Technology, pp. 152-156, Mar 2011.

[101] P. U. Jepsen and B. M. Fischer, "Dynamic range in terahertz timedomain transmission and reflection spectroscopy," Opt. Lett., vol. 30, no. 1, pp. 29-31, 2005.

[102] R. Appleby and H. B. Wallace, "Standoff detection of weapons and contraband in the $100 \mathrm{GHz}$ to $1 \mathrm{THz}$ region,", IEEE Transactions on antennas and propagation, vol. 55, no. 11, pp. 2944-2956, Nov 2007.

[103] M. Tanaka, H. Hirori, and M. Nagai, "THz nonlinear spectroscopy of solids," IEEE Transactions on Terahertz Science and Technology, vol. 1, no. 1, pp. 301-312, Sept 2011.

[104] N. Khiabani, Y. Huang, and S. Yaochun, "Discussion on the main parameters of $\mathrm{THz}$ photoconductive antennas as emitters," in Proceedings of the $5^{\text {th }}$ European Conference on Antennas and Propagation, pp. 462-466, Apr 2011.

[105] J. T. Darrow, B. B. Hu, X.-C. Zhang, and D. H. Auston, "Subpicosecond electromagnetic pulses from large-aperture photoconducting antennas,", Opt. Lett., vol. 15, no. 6, pp. 323-325, 1990. 
[106] D. You, R. R. Jones, P. H. Bucksbaum, and D. R. Dykaar, "Generation of high-power sub-single-cycle 500-fs electromagnetic pulses," Opt. Lett., vol. 18, no. 4, pp. 290-292, 1993.

[107] A. Dreyhaupt, S. Winnerl, T. Dekorsy, and M. Helm, "High-intensity terahertz radiation from a microstructured large-area photoconductor," Appl. Phys. Lett., vol. 86, 121114, 2005.

[108] M. Beck, H. Schäfer, G. Klatt, J. Demsar, S. Winnerl, M. Helm, and T. Dekorsy, "Impulsive terahertz radiation with high electric fields from an amplifier-driven large-area photoconductive antenna," Opt. Express, vol. 18, no. 9, pp. 9251-9257, 2010.

[109] X. Ropagnol, R. Morandotti, T. Ozaki, and M. Reid, "THz pulse shaping and improved optical-to- $\mathrm{THz}$ conversion efficiency using a binary phase mask," Opt. Lett., vol. 36, no. 14, pp. 2662-2664, 2011.

[110] Y. Liu, S.-G. Park, and A. M. Weiner, "Enhancement of narrow-band terahertz radiation from photoconducting antennas by optical pulse shaping," Opt. Lett., vol. 21, no. 21, pp. 1762-1764, 1996.

[111] A.S. Weling and T.F. Heinz, "Enhancement in the spectral irradiance of photoconducting terahertz emitters by chirped-pulse mixing," J. Opt. Soc. Am. B, vol. 16, no. 9, pp. 1455-1467, 1999.

[112] A. M. Weiner, "Ultrafast optical pulse shaping: a tutorial review," Opt. Comm., vol. 284, pp. 3669-3692, 2011

[113] S Vidal, J. Degert, J. Oberlé, and E. Freysz, "Femtosecond optical pulse shaping for tunable terahertz pulse generation," J. Opt. Soc. Am. B, vol. 27, no. 5, pp.1044-1050, 2010.

[114] R. E. Saperstein and Y. Fainman, "Information processing with longitudinal spectral decomposition of ultrafast pulses," Appl. Opt., vol. 47, no. 4, pp. A21-A31, Feb 2008.

[115] H. Chi and J. Yao, "All-fiber chirped microwave pulses generation based on spectral shaping and wavelength-to-time conversion," IEEE Transactions on Microwave Theory and Techniques, vol. 55, no. 9, pp. 1958-1963, Sept 2007.

[116] K. Jamshidi, A. Wiatrek, C. Bersch, G. Onishchukov, G. Leuchs, and T. Schneider, "Widely tunable optical delay generator," Opt. Lett., vol. 35, no. 21, pp. 3592-3594, Nov 2010.

[117] H. Ju, S. Zhang, D. Lenstra, H. de Waardt, E. Tangdiongga, G. Khoe and H. Dorren, "SOA-based all-optical switch with subpicosecond full recovery," Opt. Express, vol. 13, pp. 942-947, 2005. 
[118] I. L. Budunoglu, C. Ülgüdür, B. Oktem, and F. Ö. Ilday, "Intensity noise of mode-locked fiber lasers," Opt. Lett., vol. 34, no. 16, pp. 25162518, Aug 2009.

[119] P. A. Elzinga, F. E. Lytle, Y. Jiang, G. B. King, and N. M. Laurendeau, "Pump/probe spectroscopy by asynchronous optical sampling," Appl. Spectrosc., vol. 41, no. 1, pp. 2-4, 1987

[120] A. Bartels, R. Cerna, C. Kistner, A. Thoma, F. Hudert, C. Janke, and T. Dekorsy, "Ultrafast time-domain spectroscopy based on high-speed asynchronous optical sampling," Rev. Sci. Instrum., vol. 78, no. 3, 035107, 2007.

[121] F. Tauser, C. Rausch, J. H. Posthumus, and F. Lison, "Electronically controlled optical sampling using $100 \mathrm{MHz}$ repetition rate fiber lasers," Proc. of SPIE, vol. 6881, pp. 68810O-68810O-8, 2008.

[122] R. Wilk, T. Hochrein, M. Koch, M. Mei, and R. Holzwarth, "OSCAT: Novel technique for time-resolved experiments without moveable optical delay lines," J. of Infrared, Millimeter and Terahertz Waves, vol. 32, no. 5, pp. 596-602, 2011.

[123] J. B. Khurgin, "Slow light in various media: a tutorial," Advances in Optics and Photonics, vol. 2, no. 3, pp. 287-318, 2010.

[124] K. Y. Song, M. G. Herráez, and L. Thévenaz, "Observation of pulse delaying and advancement in optical fibers using stimulated Brillouin scattering," Opt. Express, vol. 13, no. 1, pp. 82-88, Jan. 2005.

[125] J. E. Sharping, Y. Okawachi, and A. L. Gaeta, "Wide bandwidth slow light using a Raman fiber amplifier," Opt. Express, vol. 13, no. 16, pp. 6092-6098, Aug. 2005.

[126] Y. Okawachi, J. E. Sharping, C. Xu, and A. L. Gaeta, "Large tunable optical delays via self-phase modulation and dispersion," Opt. Express, vol. 14, no. 25, pp. 12022- 12027, Dec 2006.

[127] B. Pesala, F. Sedgwick, A. V. Uskov, and C. Chang-Hasnain, "Electrically tunable fast light at $\mathrm{THz}$ bandwidth using cascaded semiconductor optical amplifiers," Opt. Express, vol. 15, no. 24, pp. 15863-15867, 2007.

[128] M. Xin, F. Yin, C. Lei, M. Chen, H. Chen, C. Tang, and S. Xie, "Broadband tunable optical delay based on real-time Fourier transformation and ramp-type phase modulation," Opt. Lett., vol. 35, no. 10, pp. 1596-1598, May 2010. 
[129] J. Palací, G. E. Villanueva and J. Herrera, "EAM-SOA Millimeter-Wave Frequency Up-Converter for Radio-over-Fiber Applications," Optics Commun., Vol. 284, No. 1, pp. 98-102, 2011.

[130] E. Hellström, H. Sunnerud, M. Westlund, and M. Karlsson, "Third-order dispersion compensation using a phase modulator," J. Lightwave Technol., vol. 21, no. 5, pp. 1188-1197, May 2003. 


\section{Papers A-G}





\section{Paper A}

"Reconfigurable photonic microwave filter based on four-wave mixing"

B. Vidal, J. Palací, and J. Capmany 



\title{
Reconfigurable photonic microwave filter based on four-wave mixing
}

\author{
Borja Vidal $^{1}$, Jesús Palací ${ }^{1}$ and José Capmany ${ }^{2}$ \\ ${ }^{1}$ Nanophotonics Technology Center, Universidad \\ Politécnica de Valencia, 46022 Valencia, Spain \\ ${ }^{2}$ ITEAM Research Institute, Universidad Politécnica \\ de Valencia, 46022 Valencia, Spain
}

\begin{abstract}
A simple technique to reshape the response of tapped delay line photonic microwave filters is demonstrated. The technique is based on the generation and control of a set of optical carriers by means of four-wave mixing. Experimental results show reconfiguration from 2 to 5 taps with uniform and apodized amplitude distributions.
\end{abstract}

\section{Introduction}

Photonic technology offers an alternative implementation of microwave filtering which shows attractive features over its pure electric counterparts: wide bandwidth (up to $100 \mathrm{GHz}$ ), seamless tunability, low sensitivity to electromagnetic interference, and potential of integration with radio over fiber links [1], [2]. In the last years different approaches have been proposed including infiniteimpulse response filters [3], ring resonators [4], [5], nonlinear effects in fiber [6] and finite-impulse response tapped delay line filters [7]-[12].

Among these options, delay line filters have attracted particular interest due to their flexibility to implement a wide range of functionalities such as flat-top bandpass responses based on positive and negative coefficients, fast tuning, etc. On the other side, this approach is traditionally based on using as many optical sources as filter taps which limits its scalability. To overcome this limitation, reduce cost and ease practical deployment, several methods have been studied. These include the use of optical spectrum slicing [7], which reduces the filter performance in terms of noise; and comb sources [10]-[12], which offer good performance but can require complex 
control signaling, increasing the complexity of the system. In [12] fast tuning as well as high stopband attenuation $(70 \mathrm{~dB})$ were demonstrated using comb sources combined with four-wave mixing (FWM) with the double objective of increasing the number of modes and smoothing the comb response. The former approach however does not offer, in principle, the control of the individual laser mode amplitudes and thus have restricted capabilities to dynamically control the filter response by means of tap windowing. Some schemes have addressed this issue by offering limited control or an increase in complexity [13], [14].

In this paper, an approach to increase the number of taps featuring dynamically control the filter shape based on FWM is demonstrated. By controlling the gain of an optical amplifier and the state of polarization the number of taps of the filter as well as its response shape can be controlled.

\section{Principle of operation}

The technique is based on the dynamic control of the shape and number of optical carriers (i.e., filter taps) in a taped delay line FIR filter based on a set of optical carriers and a dispersive medium by means of FWM. FWM is a parametric process given by the thirdorder electric susceptibility of the fiber [15]. In general it occurs when three waves of different wavelength $\left(v_{1}, v_{2}, v_{3}\right)$ copropagate in a fiber generating new waves whose frequencies are a combination of the original frequencies. If all the degenerate and partially degenerate processes are taken into account products at nine new frequencies are produced although the weaker frequencies are usually neglected and only the strongest frequency component at $v_{4}=v_{3}+\left(v_{2}-v_{1}\right)=v_{2}+\left(v_{3}-v_{1}\right)$, known as idler, is considered. In the particular case where only two waves of different frequency are present, case known as partially degenerated FWM, two idler waves are generated as shown in Fig. 1 although by increasing the power of the optical carriers it is possible to generate additional carriers. Thus, by controlling the power of the pump carriers (for instance using an optical amplifier) it is possible to control the number of carriers (filter taps) and their amplitude. Additionally, further control may be obtained by adding optical 
filtering after the FWM carrier generation stage to improve the control of the filter amplitude distribution.

FWM generated idler carriers can be used to increase the number of taps of a photonic microwave filter while keeping control of the relative amplitude among the carriers, i.e., controlling the shape of the filter response.

The technique does not affect the tuning of the filter response, which may be performed either by changing the wavelength spacing between the original optical carriers or changing total dispersion in the dispersive medium, as reported elsewhere [1].

\section{Experimental results}

The experimental setup is shown in Fig. 2. Two continuous-wave optical sources (one distributed feedback laser, DFB, at $1559.6 \mathrm{~nm}$ and one external cavity laser, ECL at $1560.4 \mathrm{~nm}$ ) were used as pump waves. These are amplified by a high-power erbium-doped fiber amplifier (EDFA) and applied to a reel of highly nonlinear fiber (HNLF). The HNLF used has a zero dispersion wavelength $\left(\lambda_{0}\right)$ of $1562 \mathrm{~nm}$, a dispersion slope $\mathrm{S}$ of $0.018 \mathrm{ps} /\left(\mathrm{nm}^{2} \cdot \mathrm{km}\right)$, a nonlinear coefficient $\gamma$ of $10.8 \mathrm{~W}^{-1} \cdot \mathrm{km}^{-1}$ and a length of 900 meters. At the output of the fiber FWM results in the generation of new carriers.

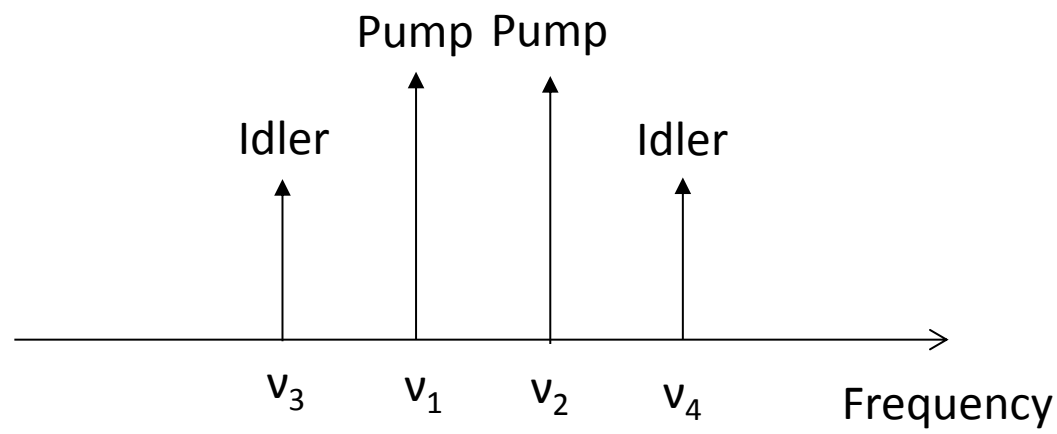

Figure 1: Schematic spectrum of frequency components generated for partially-degenerated FWM. 


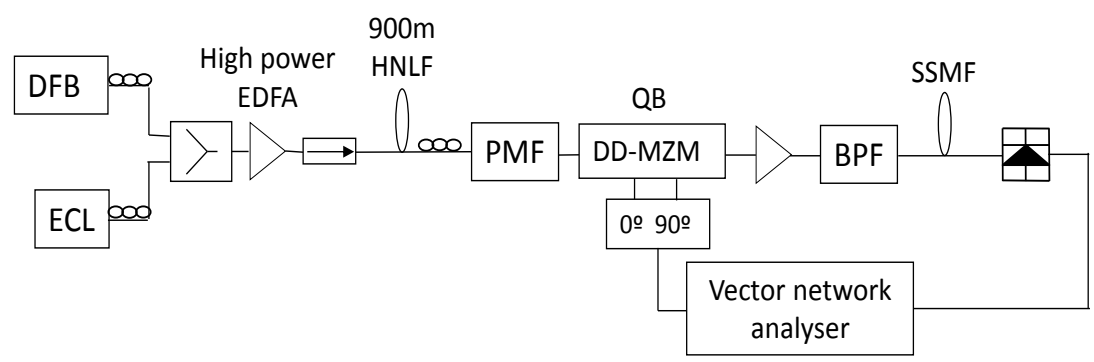

Figure 2: Experimental setup of the reconfigurable multiple tap delay line filter based on two optical carriers. PMF: Polarization maintaining fiber, BPF: bandpass filter.

The filter response is controlled by changing the number and relative amplitude of the optical carriers. It is done by changing the power of the pump carriers through the gain of the optical amplifier. Additionally, to increase control over the optical carriers, the amplitudes of the optical carriers can be adjusted by means of optical filtering [16]-[19]. A polarization controller and a short length of polarization maintaining fiber (differential group delay, DGD, of around $1.5 \mathrm{ps}$ ), is introduced after the HNLF. It results in an optical filter generated by the light travelling along the two axes of the modulator with the DGD introduced by the PMF. Fig. 3 shows the optical filter response obtained for different polarization states at the input of the PMF. As it can be seen the optical filter FSR is around $5.5 \mathrm{~nm}$ which agrees with the theoretical value $(5.4 \mathrm{~nm})$. This optical filtering stage allows further adjustment of the amplitudes of the set of optical carriers. After the filter control stage, optical carriers are modulated in a quadrature-biased (QB) Mach-Zehnder modulator, fed by the microwave signal generated by a vector network analyzer (Agilent PNA-X). To avoid dispersion induced fading a dual-drive MZM is used to get single sideband modulation (SSB). After modulation the signals are amplified and filtered to reduce noise and launched to a reel of $10 \mathrm{~km}$ standard single mode fiber (SSMF) where time delays are generated through fiber dispersion. Finally, the signals are photodetected and the filter response obtained as the $\mathrm{S}_{21}$ parameter in the vector network analyzer. 


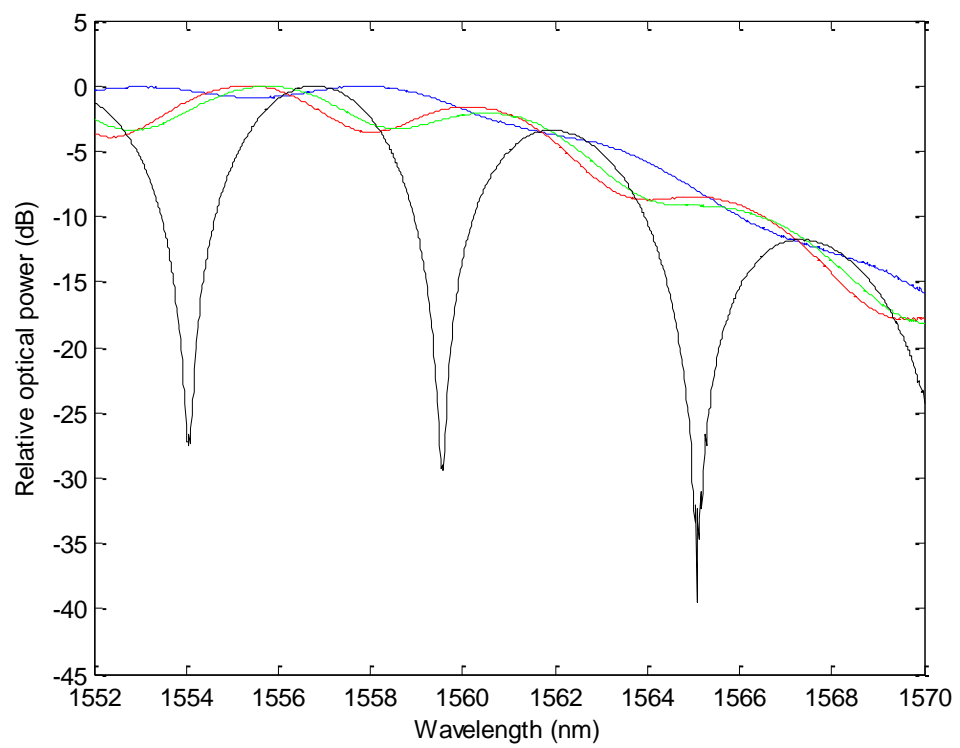

Figure 3: Some optical filter response implemented with a PMF and an external amplitude modulator for different input polarization states.

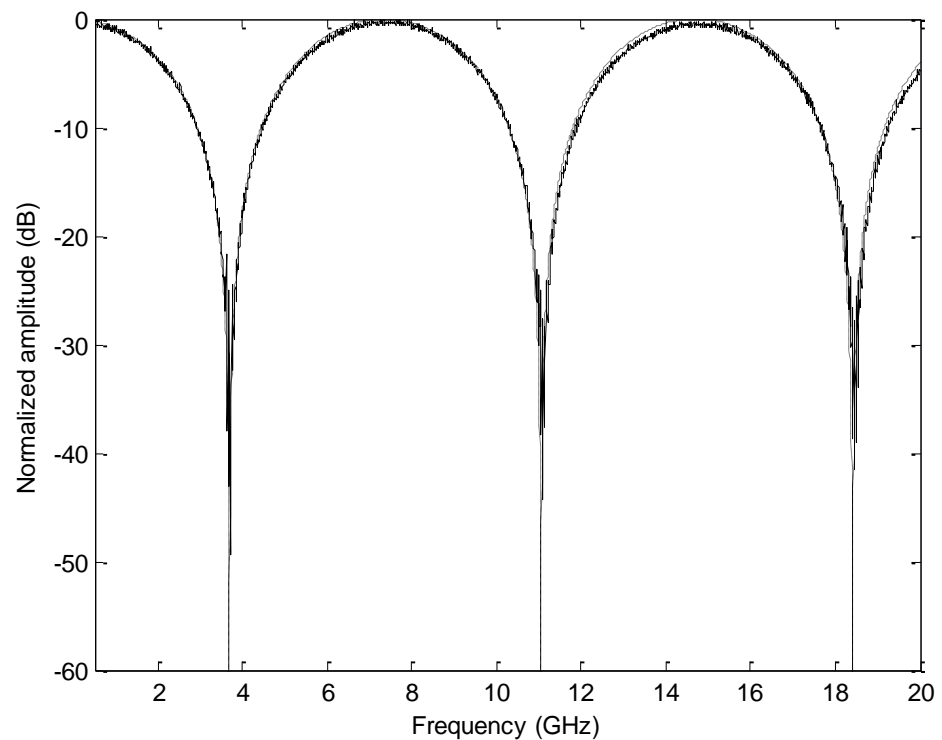

Figure 4: Notch filter response obtained with low EDFA gain. The solid curve represents experimental results and dashed one theoretical results obtained from fitted parameters. 
Controlling the gain of the high-power EDFA and the polarization at the input of the PMF it is possible to control the amplitude distribution of the optical powers and therefore reshape the filter response. Fig. 4 shows the filter response using the setup of Fig. 2 when the high-power EDFA is kept at low gain, no significant FWM is generated and a two-tap notch filter response is obtained as in a conventional tapped delay line photonic microwave filter.

If the gain of the high-power EDFA is increased, idler carriers are generated [Fig. 5(a)]. To shape the filter response the gain of the EDFA is adjusted jointly with the optical filter response generated by the combination of PMF birefringence and the polarization axis of the external modulator.

Adjusting both EDFA gain and polarization at the input of the PMF, a three-tap delay line filter can be implemented as shown in Fig. 5(a) where the optical spectrum showing SSB-modulated optical carriers is depicted. Fig. 5(a) shows how several idler waves are generated but only some of them, three in this case, have enough power to generate significant microwave taps. As it can be seen in Fig. 5(b), a uniform amplitude distribution can be obtained.

Fig. 6 shows results for a four-tap delay line filter. Both uniform and particular amplitude distributions (for instance, a Hanning window) can be obtained with the proposed technique as shown in Figs. 6 and $7(\mathrm{~b})$.

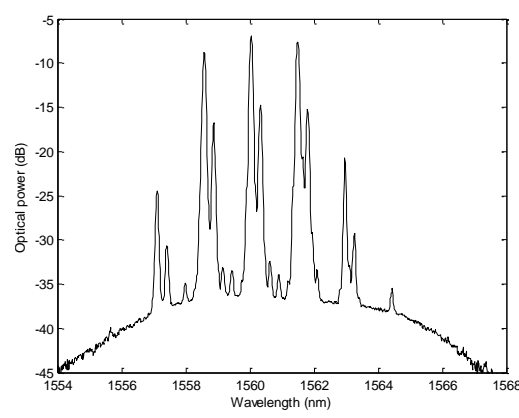

(a)

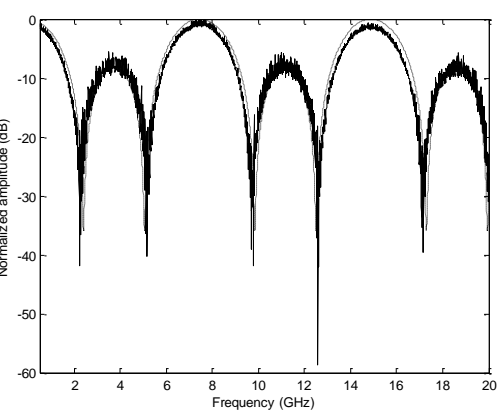

(b)

Figure 5: (a) Optical spectrum. (b) Three-tap filter response. Solid curve experimental results, dashed curve theoretical results obtained from fitted parameters. 


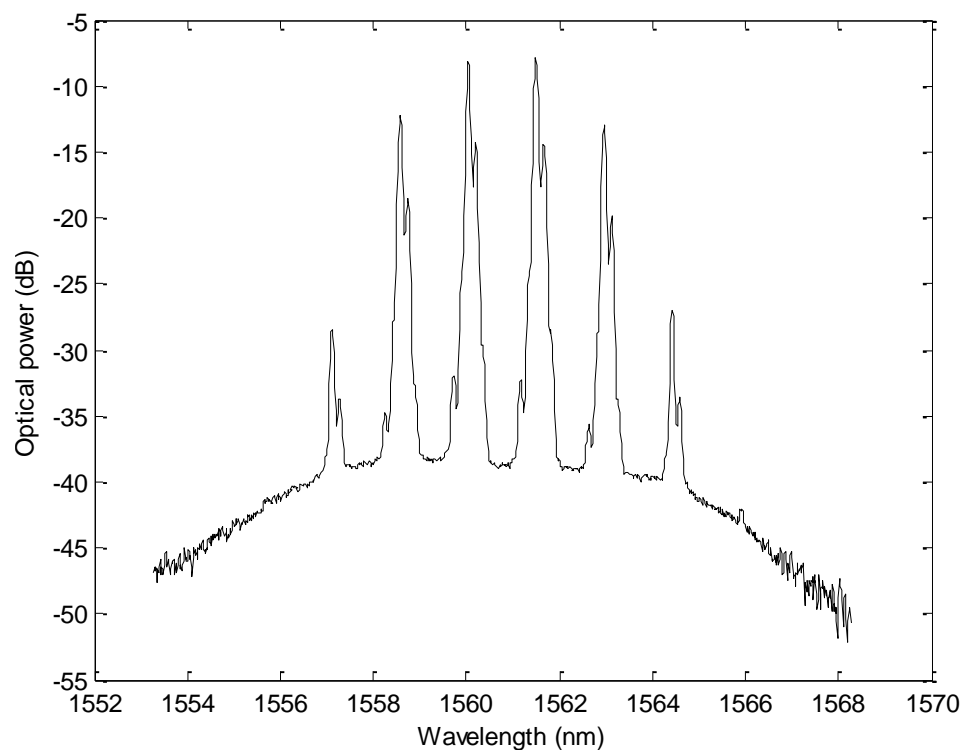

Figure 6: Optical carriers corresponding to a four-tap amplitude distribution according to a Hanning window.

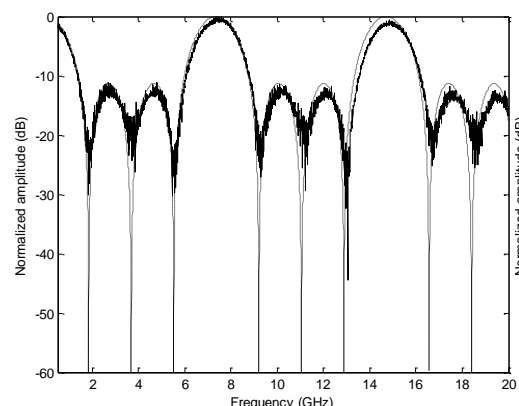

(a)

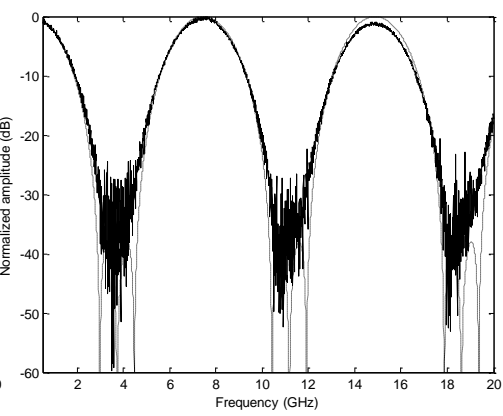

(b)

Figure 7: Four-tap filter response. (a) Uniform amplitude distribution. (b) Hanning window distribution as shown in Figure 5. Solid curves represent experimental results, and dashed curves theoretical results obtained from fitted parameters. 


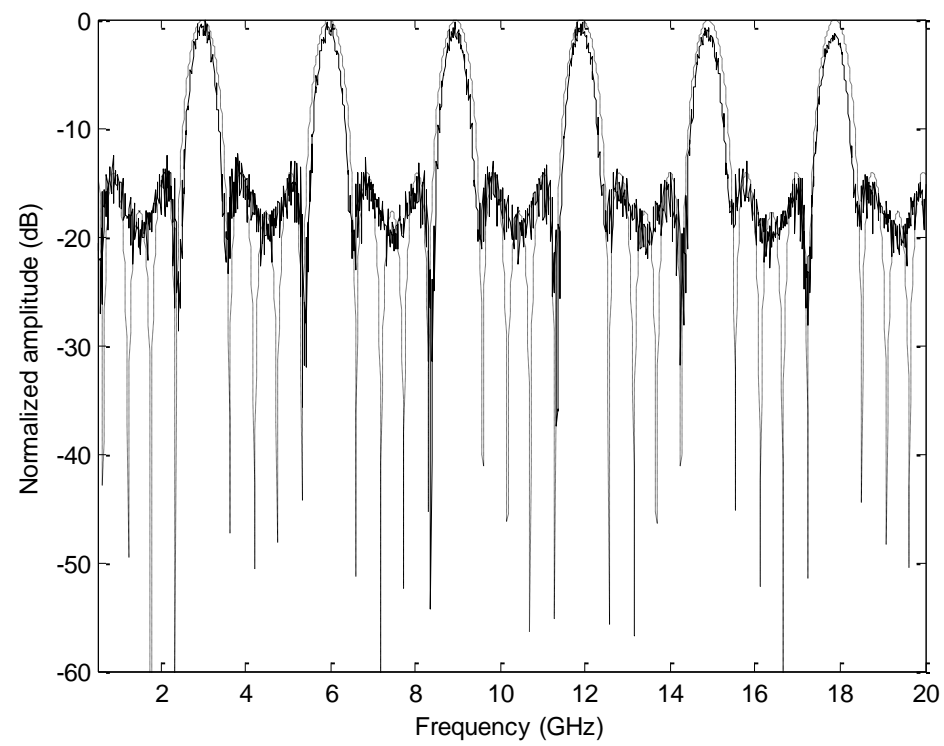

Figure 8: Five-tap filter response. Solid curve experimental results, dashed curve theoretical results obtained from fitted parameters.

Finally, a five-tap apodized filter response $\left(\left[\begin{array}{lllll}0.8 & 1 & 1 & 1 & 0.8\end{array}\right]\right)$ is obtained as shown in Fig. 8. In this case a dispersive fiber of $25 \mathrm{~km}$ was used and the filter used to reduce noise was eliminated to accommodate the FWM-generated optical carriers.

Although the proposed technique has been demonstrated for a modest number of seeding optical carriers the principle can be expanded to generate a number $\mathrm{N}$ of spectral samples starting from $\mathrm{N} / 2$ pumping laser modes.

\section{Conclusion}

An approach to dynamically reshape the transfer function of a multitap photonic microwave filter by changing the number of taps and controlling the amplitude distribution has been demonstrated. Proof-of-concept experiments have shown the dynamic reconfiguration of a photonic microwave filter response by changing both the number of taps from 2 to 5 in a setup with two optical sources and the amplitude window (uniform and Hanning). The scheme is simple and can be easily integrated in radio over fiber links. 


\section{References}

[1] J. Capmany and D. Novak, "Microwave photonics combines two worlds," Nat. Photon., vol. 1, no. 6, pp. 319-330, Jun. 2007.

[2] J. Yao, "Microwave Photonics," J. Lightw. Technol., vol. 27, no. 3, pp. 314-335, 2009.

[3] E. H. W. Chan and R. A. Minasian, "Coherence-free photonic notch filter," Electron. Lett., vol. 40, no. 21, pp. 1375-1377, Oct. 2004.

[4] M. S. Rasras, K. Y. Tu, D. M. Gill, Y. K. Chen, A. E. White, S. S. Patel, A. Pomerene, D. Carothers, J. Beattie, M. Beals, J. Michel, and L. C. Kimerling, "Demonstration of a tunable microwave-photonic notch filter using low-loss silicon ring resonator," J. Lightw. Technol., vol. 27, no. 12, pp. 2105-2110, Jun. 15, 2009.

[5] J. Palací, G. E. Villanueva, J.V. Galán, J. Martí, and B. Vidal, "Single bandpass photonic microwave filter based on a notch ring resonator," IEEE Photon. Technol. Lett., vol. 22, no. 17, pp. 12761278, Sep. 2010.

[6] B. Vidal, M. A. Piqueras, and J. Martí, "Tunable and reconfigurable photonic microwave filter based on stimulated Brillouin scattering," Opt. Lett., vol. 32, no. 1, pp. 23-25, Jan. 1, 2007.

[7] A. P. Foord, P. A. Davies, and P. A. Greenhalgh, "Synthesis of microwave and millimetre-wave filters using optical spectrum-slicing," Electron. Lett., vol. 32, no. 4, pp. 390-391, Feb. 1996.

[8] J. Capmany, D. Pastor, and B. Ortega, "New and flexible fiber-optic delay line filters using chirped fiber Bragg gratings and laser arrays," IEEE Trans. Microw. Theory Tech., vol. 47, no. 7, pp. 1321-1326, Jul. 1999.

[9] B. Vidal, J. L. Corral, and J. Martí, "All-optical WDM multi-tap microwave filter with flat bandpass," Opt. Exp., vol. 14, no. 2, pp. 581586, Jan. 23, 2006.

[10] M. Bolea, J. Mora, B. Ortega, and J. Capmany, "Highly chirped single-bandpass microwave photonic filter with reconfiguration capabilities," Opt. Exp., vol. 19, no. 5, pp. 4566-4576, Feb. 28, 2011.

[11] M. Song, C. M. Long, R. Wu, D. Seo, D. E. Leaird, and A. M. Weiner, "Reconfigurable and tunable flat-top microwave photonic filters utilizing optical frequency combs," IEEE Photon. Technol. Lett., vol. 23, no. 21, pp. 1618-1620, Nov. 2011. 
[12] V. R. Supradeepa, C. M. Long, R. Wu, F. Ferdous, E. Hamidi, D. E. Leaird, and A. M. Weiner, "Comb-based radiofrequency photonic filters with rapid tunability and high selectivity," Nat. Photon., vol. 6, no. 3, pp. 186-194, Feb. 2012.

[13] B. Vidal, M. A. Piqueras, and J. Martí, "Photonic microwave filter based on spectrum slicing with reconfiguration capability," Electron. Lett., vol. 41, no. 23, pp. 1286-1287, Nov. 10, 2005.

[14] J. Capmany, J. Mora, D. Pastor, and B. Ortega, "High-quality online-reconfigurable microwave photonic transversal filter with positive and negative coefficients," IEEE Photon. Technol. Lett., vol. 17, no. 12, pp. 2730-2732, Dec. 2005.

[15] G. P. Agrawal, Nonlinear Fiber Optics, 3rd ed., San Diego, CA: Academic, 2001.

[16] B. Vidal, T. Mengual, C. Ibáñez-López, and J. Martí, "WDM photonic microwave filter with variable cosine windowing based on a DGD module," Photon. Technol. Lett., vol. 18, no. 21, pp. 2272-2274, Nov. 2006.

[17] Q. Wang and J. Yao, "Multitap photonic microwave filters with arbitrary positive and negative coefficients using a polarization modulator and an optical polarizer," IEEE Photon. Technol. Lett., vol. 20, no. 2, pp. 78-80, Jan. 2008.

[18] H. Y. Jiang, L. S. Yan, J. Ye, W. Pan, B. Luo, and X. S. Yao, "Comb filter with independently tunable wavelength spacing and bandwidth using cascaded variable differential group delay elements," Opt. Lett., vol. 36, no. 12, pp. 2305-2307, Jun. 2011.

[19] L. S. Yan, J. Ye, H. Y. Jiang, W. Pan, B. Luo, A. L. Yi, Y. H. Guo, and X. S. Yao, "A photonic comb filter with independently and digitally tunable bandwidth and frequency spacing," IEEE Photon. Technol. Lett., vol. 23, no. 13, pp. 857-859, Jul. 2011. 


\section{Paper B}

\section{"Tunable photonic microwave filter with single bandpass based on a phase-shifted fiber Bragg grating"}

J. Palací, P. Pérez-Millán, G. E. Villanueva, J. L. Cruz,

M. V. Andrés, J. Martí and B. Vidal

IEEE Photon. Technol. Lett., Vol. 22, No. 19, pp. 1467-1469, October 2010 



\title{
Tunable photonic microwave filter with single bandpass based on a phase-shifted fiber Bragg grating
}

\author{
Jesús Palací ${ }^{1}$, Pere Pérez-Millán ${ }^{1}$, Guillermo E. Villanueva ${ }^{1}$, \\ José L. Cruz ${ }^{2}$, Miguel V. Andrés ${ }^{2}$, Javier Martí \\ and Borja Vidal ${ }^{1}$ \\ ${ }^{1}$ Nanophotonics Technology Center, Universidad \\ Politécnica de Valencia, Camino de Vera s/n, 46022 \\ Valencia, Spain \\ ${ }^{2}$ Departamento de Física Aplicada-ICMUV, \\ Universitat de València, 46100 Burjassot, Spain
}

\begin{abstract}
A novel tunable photonic microwave single bandpass filter based on the optical resonance originated by a local phase shift introduced in the periodic structure of a fiber Bragg grating is proposed. Dynamic control of the phase shift is obtained employing a piezoelectric transducer in order to stretch the grating, thus changing the resonance wavelength. A photonic microwave filter is obtained by using an optical single-sideband modulation. Experimental results are provided in order to prove the concept.
\end{abstract}

\section{Introduction}

Photonic technology has found many applications in data transmission, sensing, bio and microwave photonics [1], especially in the microwave and millimeter-wave bands where better performance in comparison with traditional technology can be obtained. One of the most interesting areas where optical technology could provide benefits is photonic microwave filtering. Many different architectures have been proposed [2]-[5], offering advantages such as low and frequencyindependent loss or tunability and reconfiguration of the filter response. However, most optically implemented microwave filters exhibit periodic transfer functions, preventing their use in radio- 
frequency (RF) applications. Several single bandpass microwave filters have been proposed to overcome this drawback [4], [5].

One of the most employed optical filters is the fiber Bragg grating (FBG). It consists of a periodic modulation of the refractive index of the core of an optical fiber along its longitudinal axis. When a phase shift occurs into this periodic structure an optical resonance is induced, allowing the implementation of optical narrow-band filters [6] or fiber lasers [7]. The optical resonance wavelength depends on the phase shift value, thus dynamic control of this value allows the tuning of the resonance inside the FBG bandwidth.

This letter presents a novel architecture for implementing photonic bandpass microwave filters. It is based on optically filtering a single side-band (SSB) modulated signal by means of a phase-shifted fiber Bragg grating (PSFBG). Since the optical filter is employed in transmission, an optical circulator is not required that reduces loss and complexity. The proposed scheme induces a dynamic phase shift employing a plumbum zirconate titanate (PZT) piezoelectric actuator, allowing for continuous tunability of the induced resonance and therefore the microwave filter response. Since the proposed scheme employs a PZT actuator to induce the phase-shift it exhibits benefits in terms of speed (less than $1 \mathrm{~ms}$ according to the PZT specifications) and simplicity in comparison with previously reported architectures based on temperature control [8] or changes in the index of refraction induced by means of an additional optical signal [9]. Although employing a tunable laser to tune the microwave response is not required, and using fixed wavelength lasers could reduce the complexity of the system, tuning the emission wavelength of the laser would provide more flexibility to the system.

\section{Filter operating principle}

The operating principle of the proposed filter is based on optically filtering a SSB-modulated microwave signal. The emission wavelength of a tunable laser source (TLS) is chosen to fall close to the notch optical response of an FBG. Therefore, frequencies falling in the notch response will be attenuated. If a phase shift is created by a local variation of the period and the refractive index of the FBG, an optical resonance appears into the notch response at a wavelength determined 
by the value of the accumulated phase shift induced in the grating. Considering a realistic scenario where the period suffers an harmonic change due to the stretching of the grating [10] as illustrated in Fig. $1(\mathrm{a})$

$$
\Delta \Lambda(z)=\Delta \Lambda_{\max } \sin \left(\frac{\pi}{l} z\right)
$$

where $\Delta \Lambda_{\max }$ stands for the maximum difference between the longest stretched period and the original period $\Lambda_{0}, 1$ is the length of the perturbation, and $\mathrm{z}$ the position into the perturbation. From (1) the accumulated phase shift of the periodic structure can be demonstrated to be as follows [10]:

$$
\phi=4 \pi\left(\frac{\Delta \Lambda_{\max }}{\left(\Lambda_{0} \pi+2 \Delta \Lambda_{\max }\right) \Lambda_{0}}\right) l
$$

Once the phase shift is induced by stretching of the fiber, microwave frequencies falling in the resonance will not be attenuated, thus a microwave bandpass filter will be obtained after photodetection. Given a certain phase shift and perturbation length the resonance condition can also be calculated employing (2) and the coupled mode theory.

Moreover, if a photodiode (PD) having a bandwidth smaller than the FBG full-width at half bandwidth (FWHM) is employed a microwave single bandpass filter will be obtained. Fig. 1(b) summarizes the operating principle of the proposed filter.

(a)
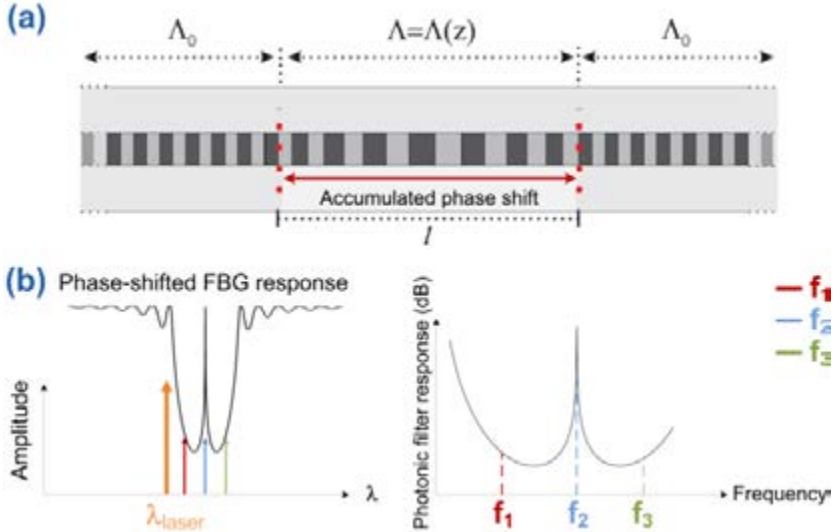

Figure 1: (a) Phase-shifted grating profile. (b) Filter operating principle: grating response with indication of three SSB modulated signals at frequencies $\mathrm{f}_{1}, \mathrm{f}_{2}$ and $\mathrm{f}_{3}$; and filter response after photo-detection. 


\section{Experimental setup}

Fig. 2 shows the scheme of the proposed filter. An optical carrier is injected to a dual-drive Mach-Zehnder modulator (MZM) feed by the microwave signal to be filtered. To obtain a SSB modulation the MZM was quadrature biased and an electrical hybrid was required to feed the arms of the MZM with a $\pi / 2$ shift. The obtained SSB signal is applied to the PSFBG, where a piezoelectric actuator was fixed to the grating. By applying a dc voltage to the PZT actuator its size increases, stretching the fiber and inducing an accumulated phase shift.

Fig. 3(a) illustrates the response of the PSFBG in transmission. Due to the optical spectrum analyzer resolution of $10 \mathrm{pm}$ the actual width of the resonance could not be measured. However, a FWHM of $0.15 \mathrm{~nm}$ for the grating can be extracted, thus a tuning bandwidth of 18.75 GHz for the bandpass filter is obtained. In Fig. 3(b), the simulated spectrum obtained by means of the coupled mode theory is depicted.

The photonic microwave filter implemented was measured employing a network analyzer. A commercially available PZT actuator was glued to the center of the FBG as stretcher. Electrical amplification was placed after photodetection in order to stay over the noise floor of the measuring device. The results obtained are included in Fig. 4 where the microwave bandpass filter obtained exhibits a FWHM of $130 \mathrm{MHz}$ and an extinction ratio (ER) close to $15 \mathrm{~dB}$. The tunability of the filter is performed changing the dc voltage applied to the stretcher. An inset illustrates the existing linear dependence between the peak frequency of the implemented bandpass filter as a function of the dc voltage for a laser wavelength of $1541.6 \mathrm{~nm}$. When a $20.39-\mathrm{V}$ voltage is applied to the actuator the stress suffered by the whole grating is the same, thus no accumulated phase shift is induced and the filter disappears. In Fig. 4, the bandpass filter exhibits two peaks as it is clearly appreciated for the filter centered at $2.6 \mathrm{GHz}$. This is caused by birefringence in the FBG, since a difference in the effective refractive indexes appears between orthogonal polarizations of the optical field. The wavelength of the optical resonance depends on the accumulated phase shift induced by stretching the grating, thus a change in the index of refraction means different resonance conditions. This originates two peaks in one of the photonic microwave responses 
included in Fig. 4. To obtain just one peak a polarization controller could be included before the PSFBG, so the linearly polarized optical field coming from the MZM is adjusted to fit one of the optical axis of the fiber.

The frequency range for tuning the microwave filter is limited by the FBG bandwidth, which could be increased employing gratings with special apodization profiles [11], [12]. The ER of the microwave bandpass filter depends on the depth of the resonance, since this parameter determines how much attenuation is introduced to the optical sideband for frequencies falling out of the optical resonance induced in the FBG. However, the ER will never be greater than the square of the power difference between the optical carrier and the ideally suppressed sideband of the SSB modulation, since the beating between these components will originate a certain electrical power at the output of the PD independent of the beating between the other sideband and the optical carrier. The width of the microwave single bandpass filter is determined by the length of the perturbation induced in the grating, thus employing shorter stretching lengths narrower bandpass filters could be obtained. However, as it is inferred from (2), considering a constant phase shift to shorten the stretching length would mean higher dc voltages are required to increase the stretching of the fiber. Thus, employing small piezoelectric actuators with high-voltage sources would lead to the implementation of tunable microwave bandpass filters with narrower bandpass bandwidths.

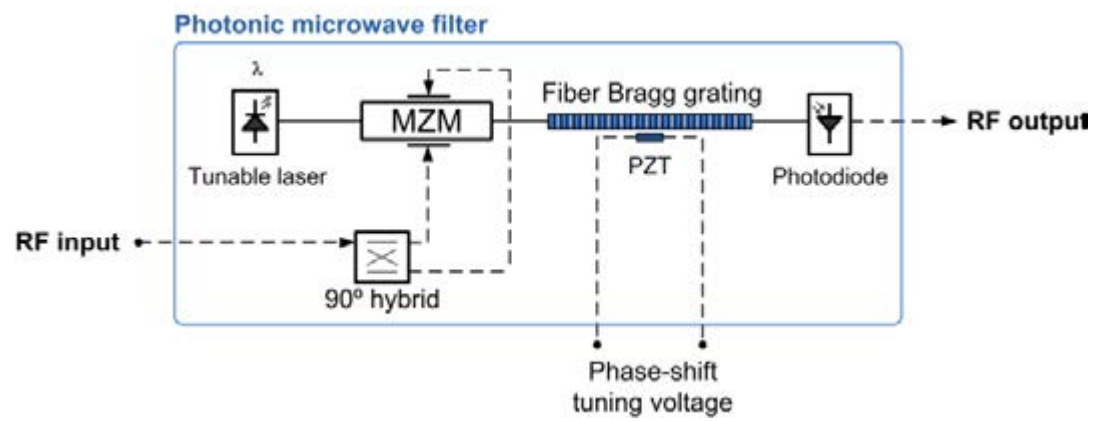

Figure 2: Scheme of the proposed photonic microwave filter. 

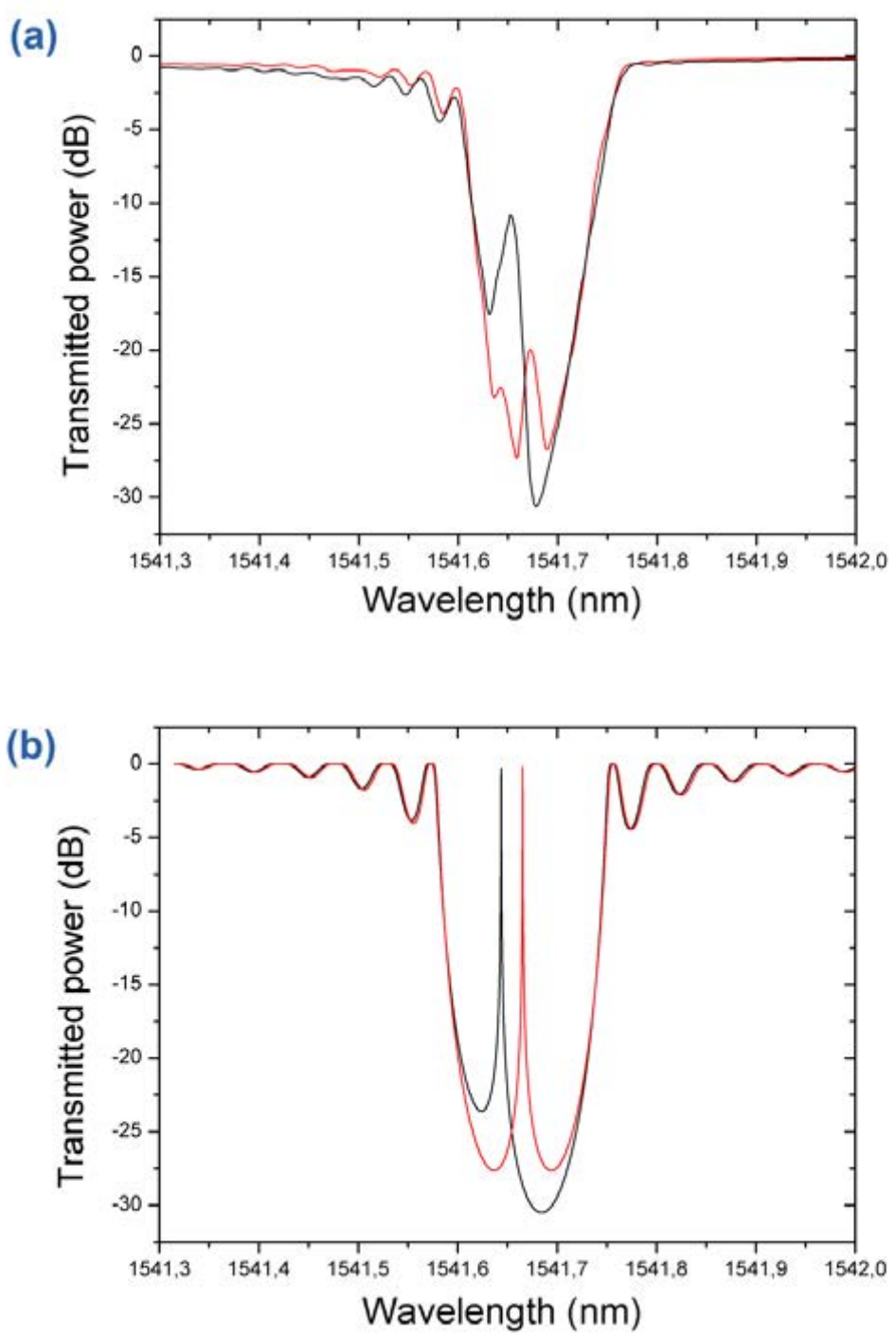

Figure 3: (a) Measured and (b) simulated transmittance spectra for the considered PSFBG. Phase shifts of $4 \pi / 5$ (black lines) and $\pi$ (red lines) are represented. 


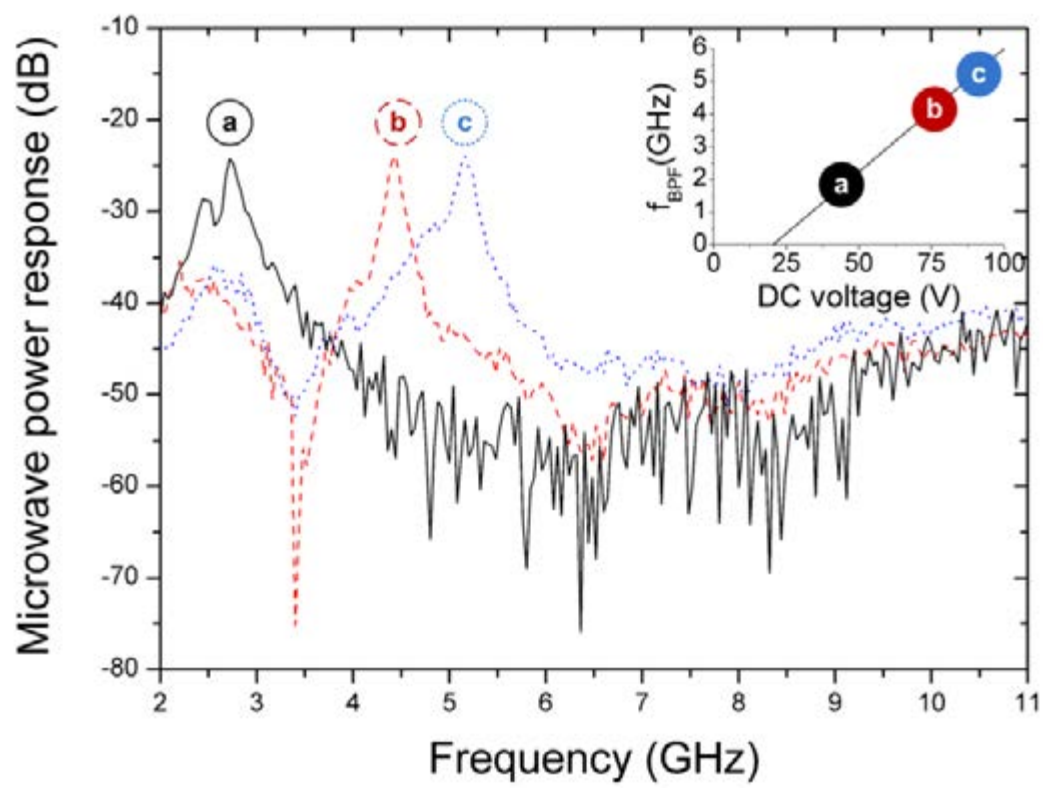

Figure 4: Photonic microwave filter response for several values of dc voltage applied to PZT actuator.

\section{Conclusion}

A tunable photonic microwave single bandpass filter implemented by means of optically filtering a single sideband signal employing a phaseshifted FBG has been proposed. A piezoelectric actuator induces a phase shift by stretching the fiber, thus dynamic tuning of the microwave filter response is achieved controlling the voltage applied to the stretching device. The piezoelectric actuator also provides important benefits in terms of speed and simplicity in comparison with changing temperature or the index of refraction when employing another optical signal.

The performance of the implemented microwave filter depends on the grating bandwidth as well as its reflectivity, thus employing highindex contrast FBGs is recommended. The size of the perturbation induced by the stretcher is also of importance, since it determines the width of the microwave bandpass filter. Although employing shorter perturbation lengths would allow for narrower responses, higher voltages feeding the piezoelectric actuator would be required. Experimental results proving the concept are provided. The tunability 
of the system is also illustrated and good performance for the implemented microwave filter is observed.

\section{References}

[1] J. Yao, "Microwave photonics," J. Lightw. Technol., vol. 27, no. 3, pp. 314-335, Feb. 1, 2009.

[2] J. Mora, B. Ortega, M. V. Andrés, J. Capmany, J. L. Cruz, D. Pastor, and S. Sales, "Tunable all-optical negative multi-tap microwave filters based on uniform fiber Bragg gratings," Opt. Lett., vol. 28, pp. 1308-1310, 2003.

[3] B. Vidal, V. Polo, J. L. Corral, and J. Martí, "Harmonic suppressed photonic microwave filter," J. Lightw. Technol., vol. 21, no. 12, pp. 3150-3154, Dec. 2003.

[4] S. Xiao and A. M. Weiner, "Coherent photonic processing of microwave signals using spatial light modulators: Programmable amplitude filters," J. Lightw. Technol., vol. 24, no. 7, pp. 2523-2529, Jul. 2006.

[5] B. Vidal, M. A. Piqueras, and J. Martí, "Tunable and reconfigurable photonic microwave filter based on stimulated Brillouin scattering," Opt. Lett., vol. 32, no. 1, pp. 23-25, Jan. 1, 2007.

[6] G. P. Agrawal and S. Radic, "Phase-shifted fiber Bragg gratings and their application for wavelength demultiplexing," IEEE Photon. Technol. Lett., vol. 6, no. 8, pp. 995-997, Aug. 1994.

[7] G. E. Villanueva, P. Pérez-Millán, J. Palací, J. L. Cruz, M. V. Andrés, and J. Martí, "Dual-wavelength DFB Erbium-doped fiber laser with tunable wavelength spacing," IEEE Photon. Technol. Lett., vol. 22, no. 4, pp. 254-256, Feb. 2010.

[8] L. Li, A. Schlzgen, X. Zhu, J. V. Moloney, J. Albert, and N. Peyghambarian, "1 $\mathrm{w}$ tunable dual-wavelength emission from cascaded distributed feedback fiber lasers," Appl. Phys. Lett., vol. 92, Mar. 2008, paper 051111.

[9] R. J.Williams, N. Jovanovic, G. D. Marshall, and M. J.Withford, "Optically switched erbium fibre laser using a tunable fibre-Bragg grating," in Proc. SPIE, vol. 7580, pp. 75800P-1-75800P1-8.

[10] P. Pérez-Millán, "Optical fiber Bragg gratings: Fiber lasers and microwave photonics," Ph.D. dissertation, Universitat de València, Valencia, Nov. 2008. 
[11] K. Hashimoto, M. Shiozaki, K. Murashima, T. Iwashima, T. Shibata, and A. Inoue, "Group delay flattened multi-channel FBG for band separation," in Proc. Eur. Conf. Optical Communication ECOC'02., Copenhagen, Denmark, Sep. 2002, vol. 4, pp. 1-2.

[12] M. Ibsen, R. Feced, P. Petropoulos, and M. N. Zervas, "99.9\% reflectivity dispersion-less square-filter fibre Bragg gratings for high speed DWDM networks," in Proc. Optical Fiber Communication Conf., Baltimore, MD, Mar. 2000, pp. 230-232. 



\section{Paper C}

"Single bandpass photonic microwave filter based on a notch ring resonator"

J. Palací, G. E. Villanueva, J. V. Galán, J. Martí and B. Vidal

IEEE Photon. Technol. Lett., Vol. 22, No. 17, pp. 1276-1278, September 2010 



\title{
Single bandpass photonic microwave filter based on a notch ring resonator
}

\author{
Jesús Palací, Guillermo E. Villanueva, José V. Galán, \\ Javier Martí and Borja Vidal \\ Nanophotonics Technology Center, \\ Universidad Politécnica de Valencia, Camino \\ de Vera s/n, 46022 Valencia, Spain
}

\begin{abstract}
A novel tunable single bandpass photonic microwave filter is proposed. It is based on optically filtering one of the sidebands of a phase-modulated optical carrier by means of the notch response of a silicon-on-insulator ring resonator. The filter response can be tuned by changing the laser wavelength. Experimental results to prove the concept are provided.
\end{abstract}

\section{Introduction}

Optical technology can be used to improve the performance of microwave and millimeter-wave systems [1]. Among the different areas where photonics could provide benefits to microwave technology, photonic microwave filters have attracted considerable interest. Different optical implementations of photonic microwave filters have been proposed [2]-[10]. This alternative approach to microwave filtering offers benefits such as low and spectrally constant loss, electromagnetic immunity as well as reconfiguration and tunability. However, photonic microwave filtering has to overcome a few issues to get closer to market. One of them is the need of single bandpass responses as conventional finite impulse response photonic microwave filters have periodic filter transfer functions. That prevents their use in practical radio-frequency $(\mathrm{RF})$ applications. Therefore proposals to reduce this effect have been proposed [6]-[8]. Another important issue is cost and size, and work is being carried out to reduce them through the implementation of photonic filtering structures compatible with complementary metal-oxide semiconductor (CMOS) silicon-based photonic integration [9]. 
This letter presents a photonic microwave filter based on a phase modulator and the use of a notch ring resonator to implement a single bandpass filter. This approach will allow the implementation of very compact, low-cost, low-consumption, integrated photonic microwave filters in a silicon chip.

\section{Filter operating principle}

Given an optical carrier injected to a phase modulator (PM) fed by an RF signal, it is well known that the generated sidebands are out of phase. The optical field at the output of the phase modulator under a small signal model is described by

$$
E_{P M}(t)=E_{0} \cdot J_{0}\left(m_{P M}\right) e^{j \omega_{0} t}+E_{0} \cdot J_{1}\left(m_{P M}\right) e^{j\left(\omega_{0}-\omega_{R F}\right) t}-E_{0} \cdot J_{1}\left(m_{P M}\right) e^{j\left(\omega_{0}+\omega_{R F}\right) t}
$$

where $E_{0}$ is the optical field of the source, $\omega_{0}$ and $\omega_{\mathrm{RF}}$ are the angular frequency of the optical carrier and $R F$ signal, respectively, $J_{n}(\cdot)$ is the nth-order Bessel function of the first kind, and $\mathrm{m}_{\mathrm{PM}}$ is the phase modulation index. If the modulated signal is applied to a photodetector $(\mathrm{PD})$, due to the mentioned -phase difference, no RF signal is obtained. However, this would not occur if one of these bands could be removed. This is the operating principle on which the proposed filter is based.

Fig. 1(a) illustrates the scheme of this filter, while Fig. 1(b) summarizes its principle of operation. An optical carrier is phasemodulated by an RF signal. The output field is then applied to an optical notch filter, in such a way that when only one of the sidebands is removed, the RF signal will be obtained at the output of the PD. Since this will only happen for frequencies falling in the notch peak, an electrical bandpass filter is obtained.

In this way, a bandpass photonic microwave filter is implemented from an optical notch structure reducing the complexity of the system. Moreover, if an optical filter with a free spectral range (FSR) higher than the PD bandwidth is employed, we can consider this microwave filter to be single bandpass. Although other structures could be used, silicon-on insulator (SOI) ring resonators would be suitable due to its notch behavior and high FSR when designed with small radius. It must be highlighted that all the devices could be integrated on a single photonic integrated circuit (PIC). 
(a)

Photonic filter

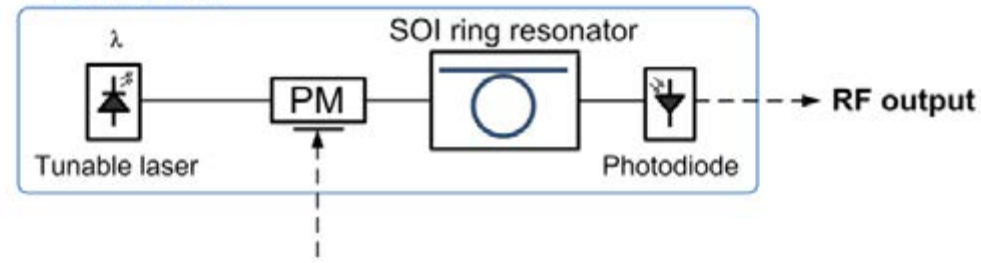

RF input

(b)
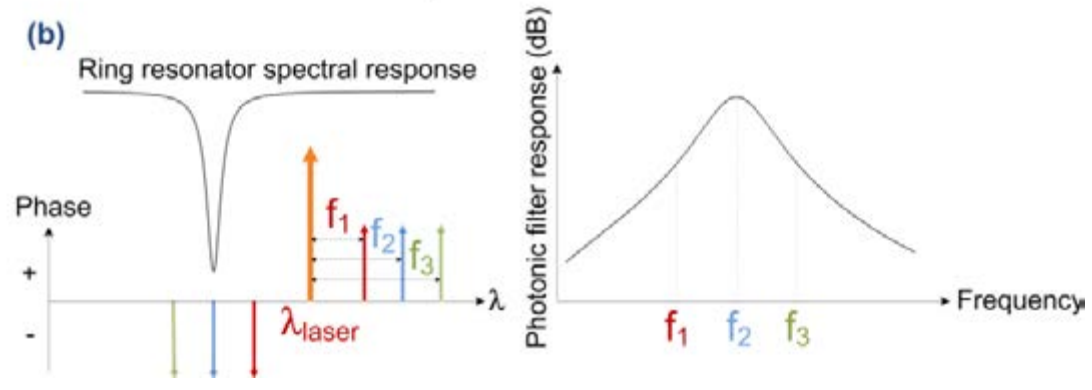

Figure 1: (a) Scheme of the proposed filter. (b) Ring resonator spectral response with the optical spectra at the output of the phase-modulator in the case of some electrical frequencies. The photonic filter response is also shown.

\section{Experimental results}

Experiments have been carried out to show the feasibility of the proposed architecture. A ring resonator was designed with a $20-\mu \mathrm{m}$ radius, where the gap between the waveguide and the ring is $200 \mathrm{~nm}$ because of the desired ring FSR and the rest of the parameters. The wavelength response of the ring was measured employing a tunable laser source (TLS). As Fig. 2 illustrates, an FSR greater than $4 \mathrm{~nm}$ was obtained, meaning that the response of the filter could be considered single bandpass at frequencies up to $250 \mathrm{GHz}$.

The setup shown in Fig. 3 was employed to measure the filter response. The bandwidth of the available phase modulator was only 10 $\mathrm{GHz}$. The network analyzer employed to sweep the RF frequency was also limited in bandwidth to $8.5 \mathrm{GHz}$, so the optical carrier had to be placed close to the resonance. In this case, it was placed at $1551.32 \mathrm{~nm}$ on the right side of the peak. Losses occurring in the alignment stage and propagation inside the SOI ring introduced $35 \mathrm{~dB}$ of attenuation. Almost $30 \mathrm{~dB}$ of these losses are caused by the inefficient process of 
coupling light from an optical fiber to SOI waveguides. By means of erbium-doped fiber amplifiers (EDFAs), $27 \mathrm{dBm}$ of optical power was achieved after the PM in order to obtain enough power at the input of the PD.

Fig. 4 shows one of the ring resonances. Due to the existing dependence between the refractive index in silicon and the optical power travelling through the waveguide, a power as high as $27 \mathrm{dBm}$ at the input of the ring resonator shifted the resonance wavelength more than $250 \mathrm{pm}$ when compared with Fig. 2. By fitting the resonance with a Lorentzian curve, the full-width at half-maximum (FWHM) and Q-factor values were $144 \mathrm{pm}$ and 10 770, respectively. Despite these good values, only about $10 \mathrm{~dB}$ of extinction ratio (ER) were obtained. From this measurement, a 3-dB bandwidth of $18 \mathrm{GHz}$ with an ER near $20 \mathrm{~dB}$ is expected for the microwave photonic filter.

The measured photonic filter response is illustrated in Fig. 5(a), as well as the estimated filter response from the resonance of Fig. 2 and its Lorentzian fit. Due to the limited frequency range which we were able to measure, only the rising slope of the filter was obtained, with an ER of almost $20 \mathrm{~dB}$ as expected. All the curves show the same behavior, although at high frequencies the measured spectra falls in comparison to the estimated ones due to the limited bandwidth of both PD and PM. The PD employed had an electrical amplifier placed after the PIN photodiode, whose gain was not flat over the full range of frequencies considered.

Although it was not possible to measure the whole filter spectra, it was estimated from optical measurements and illustrated in Fig. 5(b). An inset included in this figure shows the estimated responses when employing the Lorentzian fitting curves, where it must be stated that the ER is higher due to the absence of ripple at both sides of the peak. The estimated filter responses were obtained as follows. The optical signal at the output of the phase-modulator when applying an RF tone was obtained according to (1). Considering a laser wavelength emission of $1551.32 \mathrm{~nm}$, loss for each wavelength can be obtained from Fig. 4. Finally, the photodetection process was carried out so the electrical power at each considered frequency, from 1 to $100 \mathrm{GHz}$, was obtained. In order to illustrate the tunability of the system, two more wavelengths were considered as Fig. 5(b) shows. In order to avoid the ripple out of the bandpass, antireflective coating at both the input and 
output of the SOI ring resonator could be employed, suppressing the Fabry-Pérot response introduced by existing reflections at these accesses.

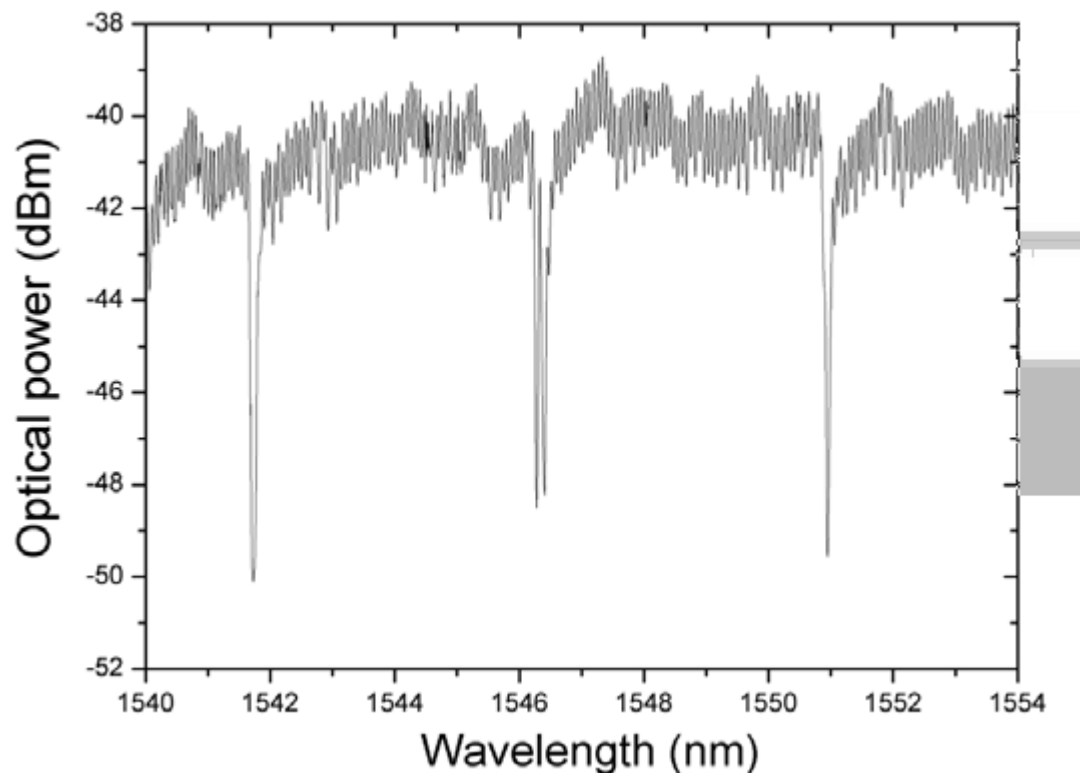

Figure 2: Experimental response of the designed SOI ring resonator as a function of the wavelength.

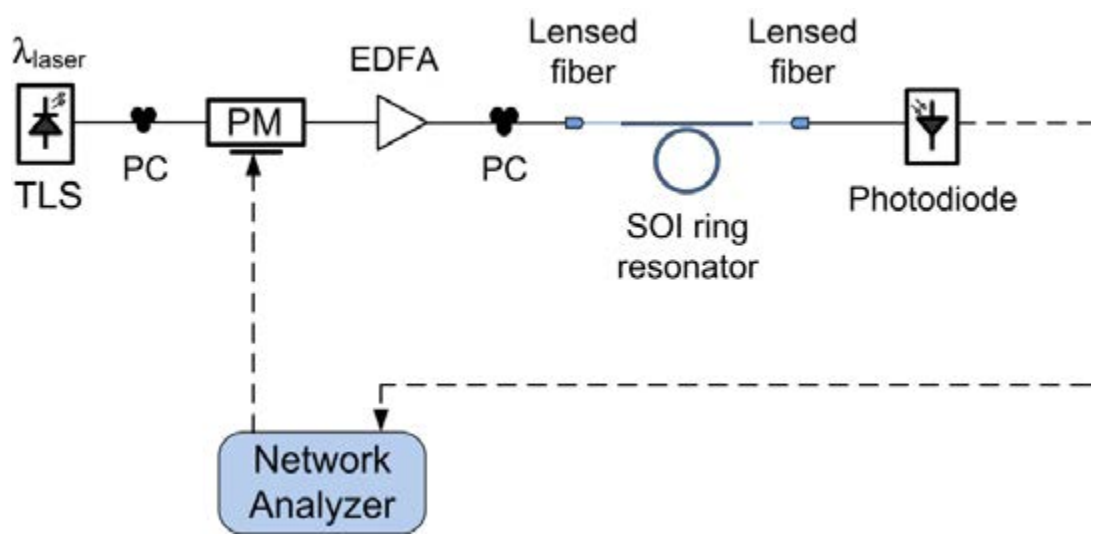

Figure 3: Setup employed while measuring the filter response. 


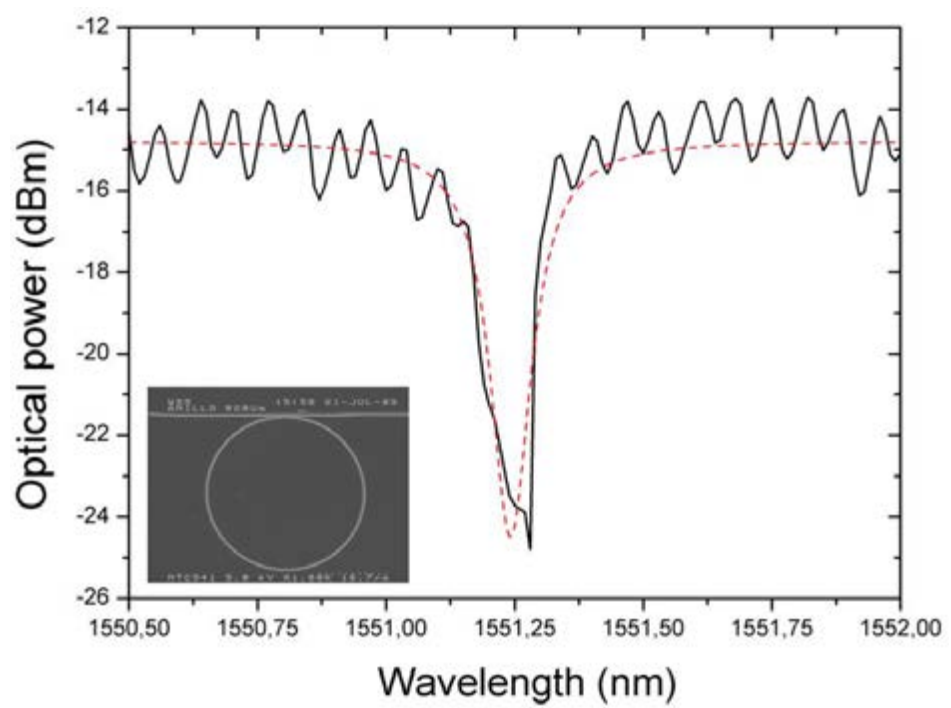

Figure 4: One of the measured resonances. A Lorentzian curve is also included (dashed line), fitting the experimental measurements. Inset: scanning electron microscope (SEM) image of the ring resonator.

The stability of the tunable laser is one important issue to be taken into account, since to implement a stable photonic microwave filter, a stable wavelength reference is needed. The tunable laser employed had $5 \mathrm{pm} / \mathrm{h}$ and $0.01 \mathrm{~dB} / \mathrm{h}$ of wavelength and power stability, respectively. Another important issue is the stability of the SOI ring resonator. The optical notch response of the ring could drift in wavelength due to changes in temperature since in silicon the index of refraction depends on this parameter. Changes in optical power when high power signals are used also result in wavelength drifts. Many solutions are available in order to provide stability to the system, for example including a Peltier cell or dynamically controlling the refractive index of silicon by means of Kerr effect by applying an electrical field.

Improving the ring resonator design and its fabrication process would provide better filter finesse and ER. To date, SOI ring resonators with a Q-factor exceeding 130.000 have been demonstrated [11], giving filter widths smaller than $1 \mathrm{GHz}$. If toroid microcavities are employed instead of a ring resonator [12], Q-factors as high as 100 million would be obtained resulting in filter bandwidths smaller than 1 $\mathrm{MHz}$. 

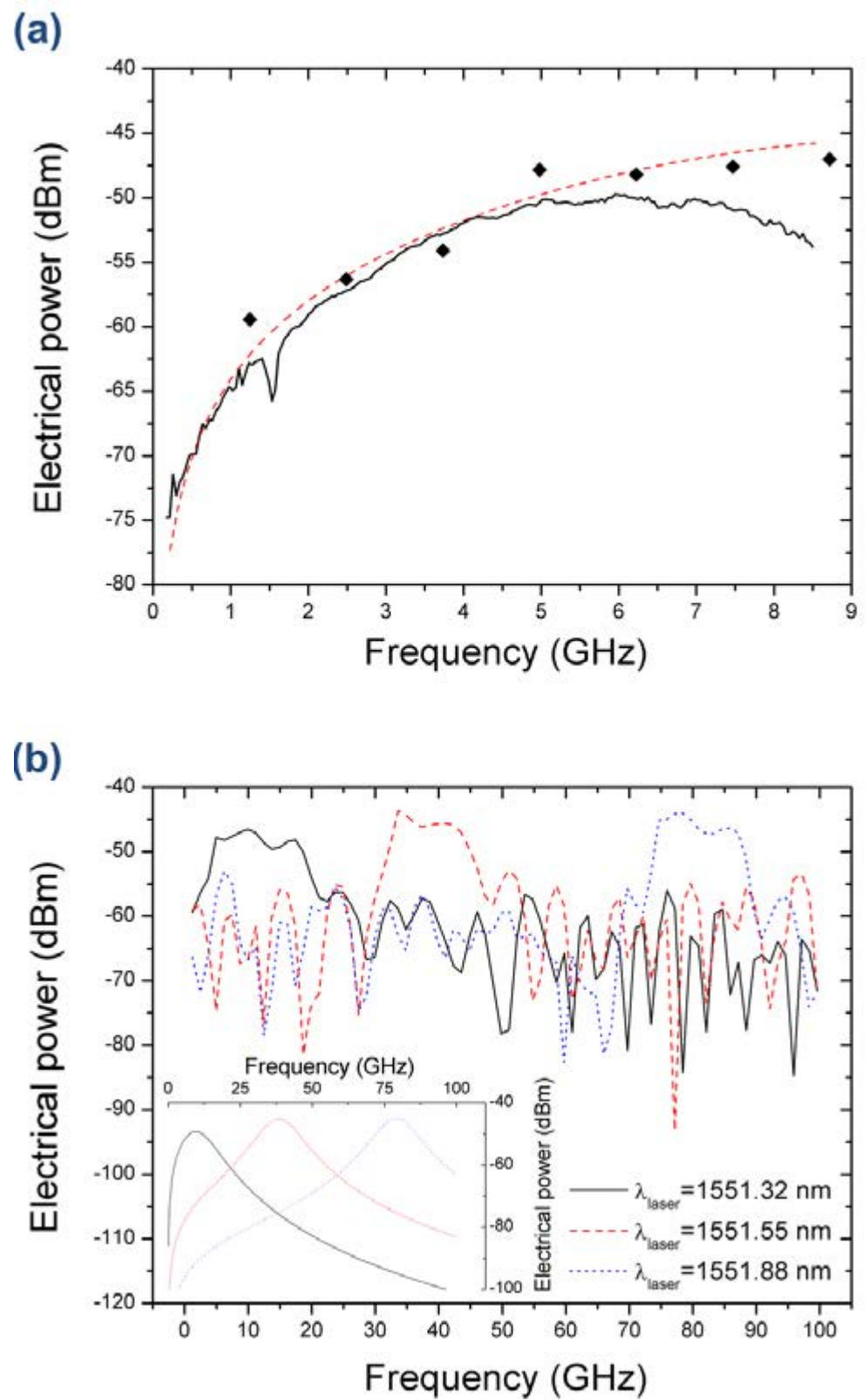

Figure 5: (a) Electric spectra of the photonic filter for a frequency range between $300 \mathrm{kHz}$ and $8.5 \mathrm{GHz}$. Continuous line is for the measured response, while scattered and dashed lines are for the estimated responses employing the measured resonance and its Lorentzian fitting, respectively. (b) Estimated filter response by varying the optical carrier frequency. The estimated response employing the Lorentzian fitting is also included as an inset. 


\section{Conclusion}

A tunable single bandpass photonic microwave filter implemented from a notch optical structure has been proposed. It is based on filtering one of the sidebands generated when phase-modulating an optical carrier signal by means of the notch response of an SOI ring resonator. The proposed filter can be considered single bandpass up to $250 \mathrm{GHz}$, and its frequency is easily tuned when changing the emission wavelength of the laser. Moreover, the whole system could be integrated on a single chip providing low-cost and low-size photonic microwave filters.

\section{References}

[1] J. Yao, "Microwave photonics," J. Lightw. Technol., vol. 27, no. 3, pp. 314-335, Feb. 1, 2009.

[2] B. Vidal, V. Polo, J. L. Corral, and J. Martí, "Photonic microwave filter with tuning and reconfiguration capabilities using optical switches and dispersive media," Electron. Lett., vol. 39, no. 6, pp. 547-549, Mar. 2003.

[3] E. H. W. Chan and R. A. Minasian, "Coherence-free photonic notch filter," Electron. Lett., vol. 40, no. 21, pp. 1375-1377, Mar. 2004.

[4] B. Vidal, M. A. Piqueras, and J. Martí, "Photonic microwave filter based on spectrum slicing with reconfiguration capability," Electron. Lett., vol. 41, no. 21, pp. 1286-1287, Nov. 2005.

[5] B. Vidal, T. Mengual, C. Ibáñez-López, and J. Martí, "WDM photonic microwave filter with variable cosine windowing based on a DGD module," IEEE Photon. Technol. Lett., vol. 18, no. 21, pp. 22722274, Nov. 1, 2006.

[6] B. Vidal, V. Polo, J. L. Corral, and J. Martí, "Harmonic suppressed photonic microwave filter," J. Lightw. Technol, vol. 21, no. 12, pp. 3150-3154, Dec. 2003.

[7] B. Vidal, M. A. Piqueras, and J. Martí, "Tunable and reconfigurable photonic microwave filter based on stimulated Brillouin scattering," Opt. Lett., vol. 32, no. 1, pp. 23-25, Jan. 2007.

[8] X. Yi and R. Minasian, "Microwave photonic filter with single bandpass response," Electron. Lett., vol. 45, no. 7, pp. 362-363, Mar. 2009. 
[9] M. S. Rasras, K. Y. Tu, D. M. Gill, Y. K. Chen, A. E. White, S. S. Patel, A. Pomerene, D. Carothers, J. Beattie, M. Beals, J. Michel, and L. C. Kimerling, "Demonstration of a tunable microwave-photonic notch filter using low-loss silicon ring resonator," J. Lightw. Technol., vol. 27, no. 12, pp. 2105-2110, Jun. 15, 2009.

[10] T.-Y. Kim, C. K. Oh, S.-J. Kim, and C.-S. Park, "Tunable photonic microwave notch filter with negative coefficient based on polarization modulation," IEEE Photon. Technol. Lett., vol. 19, no. 12, pp. 22722274, Jun. 15, 2007.

[11] J. Niehusmann, A. Vorckel, P. H. Bolivar, T.Wahlbrink, W. Henschel, and H. Kurz, "Ultrahigh-quality-factor silicon-on-insulator microring resonator," Opt. Lett., vol. 29, no. 24, pp. 2861-2863, Dec. 2004.

[12] D. K. Armani, T. J. Kippenberg, S. M. Spillane, and K. J. Vahala, "Ultra-high-Q toroid microcavity on a chip," Nature, vol. 421, pp. 925928, 2003. 



\title{
Paper D
}

"Terahertz radiation shaping based on thirdorder dispersion and self-phase modulation in standard single-mode optical fiber"

\author{
J. Palací and B. Vidal
}

J. Infrared Milli. Terahz. Waves, Vol. 33, No. 6, pp. 605-614, April 2012 



\title{
Terahertz radiation shaping based on third-order dispersion and self-phase modulation in standard single-mode optical fiber
}

\author{
Jesús Palací and Borja Vidal \\ Nanophotonics Technology Center, Universidad \\ Politécnica de Valencia, 46022 Valencia, Spain
}

\begin{abstract}
Third-order dispersion and self-phase modulation in standard single-mode fibers are employed in a fiber-based $\mathrm{THz}$ time domain spectroscopy system for radiation shaping. Ultra-short optical pulses are converted into trains of pulses, thus shaping the $\mathrm{THz}$ radiation emitted by photoconductive antennas operating at telecom wavelengths. The proposed architecture allows narrowband and wideband $\mathrm{THz}$ emission as well as tunability of the central frequency. Since the shaping takes place in standard optical fiber the architecture could be potentially implemented without requiring any additional device. Experiments showing the principle of operation have been performed demonstrating tunability of the central frequency between 350 and $800 \mathrm{GHz}$ and bandwidth from 150 $\mathrm{GHz}$ to the full bandwidth of the system.
\end{abstract}

\section{Introduction}

Radiation generated in the sub-millimeter band is finding an increasing number of applications in different fields from astronomy and spectroscopy to communications, nondestructive material testing, imaging and biology/medical sciences $[1,2]$. However, strong challenges persist unsolved in terms of efficient and versatile generation and detection.

Notable progress has been done for signals in the lower part of this band by electrical upconversion from the microwave region with several commercial solutions being available. Quantum-cascade lasers [3] have attracted great interest because they are able to provide relatively high $\mathrm{THz}$ power. However they usually work at high frequencies, limiting their deployment to the upper part of the $\mathrm{THz}$ 
region, and require cryogenic cooling. On the other side photonicbased approaches offer reasonable power levels and flexibility to implement pulsed or continuous-wave solutions [4]. Among the different solutions existent $\mathrm{THz}$ time-domain spectroscopy (THz-TDS) systems must be highlighted due to their wide use. Recently all-fiber THz-TDS systems based on photoconductive antennas (PCAs) have been proposed to reduce size and benefit from the lower cost of components developed for the telecom industry [5].

The $\mathrm{THz}$ band presents high attenuation. However, there are some spectral windows which show reduced loss. Additionally, narrowband radiation by the synthesis of particular terahertz waveforms can concentrate the radiated power in the band of interest circumventing material saturation [6]. Several schemes, some of them related to techniques previously applied to the microwave and millimeter-wave bands [7], have been proposed such as chirped-pulse mixing (CPM) [8] and Fourier synthesis methods based on phase and/or amplitude modulation of the spectrum of ultra-short optical pulses [9].

$\mathrm{THz}$ systems providing narrowband radiation are usually based on free-space components and require additional devices in comparison to standard THz-TDS. This results in bulky and expensive setups which limit the applicability of these techniques. Here we propose shaping of the radiation generated using photo-conductive antennas through both third-order dispersion (TOD) and self-phase modulation (SPM) effects occurring in standard single-mode fiber at $1550 \mathrm{~nm}$. No additional components are needed resulting in a simple and cost-effective solution. The proposed system allows for tunability and reconfigurability of the emitted $\mathrm{THz}$ radiation by changing the accumulated dispersion and the optical pulse peak power. Preliminary experiments have been carried out to validate the feasibility of the technique.

\section{Principle of operation}

The proposed technique converts a single ultra-short optical pulse into a train of pulses by controlling the intensity of non-linear effects occurring in the same optical fiber that is used to distribute the pulse. The technique exploits the interaction between dispersion and nonlinear effects in standard fiber. 
The frequency dependence of the refractive index of a given material plays an important role in the propagation of short optical pulses since its different spectral components travel at different speeds. This results in the time-broadening of the pulse. Expanding the mode-propagation constant, $\beta$, in Taylor series around a given frequency, $\omega_{0}[10]$

$$
\beta(\omega)=n(\omega) \frac{\omega}{c}=\beta_{0}+\beta_{1}\left(\omega-\omega_{0}\right)+\frac{1}{2} \beta_{2}\left(\omega-\omega_{0}\right)^{2}+\frac{1}{6} \beta_{3}\left(\omega-\omega_{0}\right)^{3}+\ldots
$$

where $\omega$ is the angular frequency, $c$ the speed of light and $\beta_{n}$ the nthderivative of $\beta$ with respect to the frequency. While $\beta_{0}$ is a constant, $\beta_{1}$ represents the propagation of the pulse envelope at the group velocity $n_{g}$. Higher order terms represent dispersion and are responsible for pulse broadening and distortion. In standard optical fibers dispersion is mainly given by $\beta_{2}$, known as second-order dispersion (SOD) or the group-velocity dispersion (GVD), and, once it is compensated, by $\beta_{3}$ or TOD. Higher order terms can be neglected in a first approach. However they should be considered when lower-order dispersion terms are compensated and long distance fiber links based on optical sources with a bandwidth comparable to the central wavelength are implemented. It is quite common to use the dispersion parameter (D) as well as its slope (S) instead of the previously defined variables.

$$
\begin{aligned}
& D=-\frac{2 \pi c}{\lambda^{2}} \beta_{2} \\
& S=\left(\frac{2 \pi c}{\lambda^{2}}\right)^{2} \beta_{3}
\end{aligned}
$$

Being $\lambda$ the central wavelength.

In the case of high energy optical pulses, as commonly used to feed photoconductive antennas, non-linear effects in the fiber have to be considered. The propagation of optical signals through a non-linear medium is accurately described by the non-linear Schrödinger equation (NLSE) [11].

$$
\frac{\partial A}{\partial z}+\frac{\alpha}{2} A+i \frac{\beta_{2}}{2} \frac{\partial^{2} A}{\partial T^{2}}-\frac{\beta_{3}}{6} \frac{\partial^{3} A}{\partial T^{3}}=i \gamma\left(|A|^{2} A+\frac{i}{\omega_{0}} \frac{\partial}{\partial T}\left(|A|^{2} A\right)-T_{R} A \frac{\partial|A|^{2}}{\partial T}\right)
$$


$A$ refers to the slowly varying pulse envelope, $\alpha$ is the absorption coefficient, $\gamma$ is the fiber nonlinear parameter, and $T_{R}$ accounts for the Raman effect. An optical field propagating along the z-axis is considered and the time variable $\mathrm{T}$ is defined as $\mathrm{T}=\mathrm{t}-\beta_{1} \cdot \mathrm{z}$ so the frame of reference follows the pulse at its group velocity.

The nonlinear parameter is defined as

$$
\gamma=\frac{n_{2} \omega_{0}}{c A_{\text {eff }}}
$$

where $n_{2}$ is the intensity-dependent part of the refraction index and $A_{\text {eff }}$ is the effective core area. The latter depends on the modal distribution of the fiber mode and therefore on the core radius and index difference between core and cladding.

From (4) it can be seen that dispersion and non-linear effects are interrelated. Depending on the scenario one of these effects can dominate. Thus it is convenient to define lengths scales for dispersion and non-linear effects, $\mathrm{L}_{\mathrm{D}}, \mathrm{L}_{\mathrm{D}}$ and $\mathrm{L}_{\mathrm{NL}}$.

$$
\begin{gathered}
L_{D}=\frac{T_{0}^{2}}{\left|\beta_{2}\right|} \\
L_{D}^{\prime}=\frac{T_{0}^{3}}{\left|\beta_{3}\right|} \\
L_{N L}=\frac{1}{\gamma P_{0}}
\end{gathered}
$$

Being $\mathrm{T}_{0}$ the initial pulse width and $\mathrm{P}_{0}$ its peak power. These values define length thresholds from whose the dispersion and nonlinear effects become significant (i. e., for simple pulse propagation in $\mathrm{L}$ meters of fiber $\mathrm{L}<<\mathrm{L}_{\mathrm{D}}$ and $\mathrm{L}<<\mathrm{L}_{\mathrm{NL}}$ would be desirable so these effects do not have a noticeable effect on the pulse). L' ${ }_{D}$ becomes important once the accumulated SOD has been compensated.

This work aims to generate trains of short pulses with a repetition rate falling in the $\mathrm{THz}$ range thanks to the interaction between dispersion and non-linear effects. Therefore the fiber link must obey $\mathrm{L}>>\mathrm{L}_{\mathrm{NL}}, \mathrm{L}<<\mathrm{L}_{\mathrm{D}}$ and $\mathrm{L}>>\mathrm{L}^{\prime}{ }_{\mathrm{D}}$. The value of $\mathrm{L}_{\mathrm{NL}}$ inversely depends on the peak power of the optical pulse while $L^{\prime}{ }_{D}$ depends on the fiber length. The accumulated SOD can be brought close to zero, therefore 
decreasing the link $\mathrm{L}_{\mathrm{D}}$, by employing dispersion compensating fiber (DCF). It is a specialty fiber whose core radius and core-cladding index difference has been designed in such a way that it presents a dispersion parameter with opposite sign compared to that of standard fiber. The proposed shaping system is shown in Fig. 1.

First of all the optical pulses are time-stretched by means of DCF. Dispersion pre-compensation is performed because broad pulses are less sensitive to nonlinear effects due to its lower peak power, easing its distribution and avoiding degradations. These pulses are driven to both transmitter and receiver by means of an optical splitter. In the transmitter branch the optical power is controlled and pulses propagating through standard single-mode fiber (SSMF) compress again. Pulse breaking arises from the effect of the accumulated TOD once the SOD has been compensated. This process can be reinforced according to the intensity of the predominant non-linear effect, self phase modulation, which originates from the dependence of the fiber refractive index with the optical power and leads to phase-modulating the pulse, getting time-shaped and spectrum-broadened [12]. These effects allow the breaking of a pulse in a set of pulses and the control of the time difference between train pulses by means of TOD, while the number of subpulses and its amplitude are determined by the intensity of the SPM, i. e. the amplitude of the initial optical pulse.

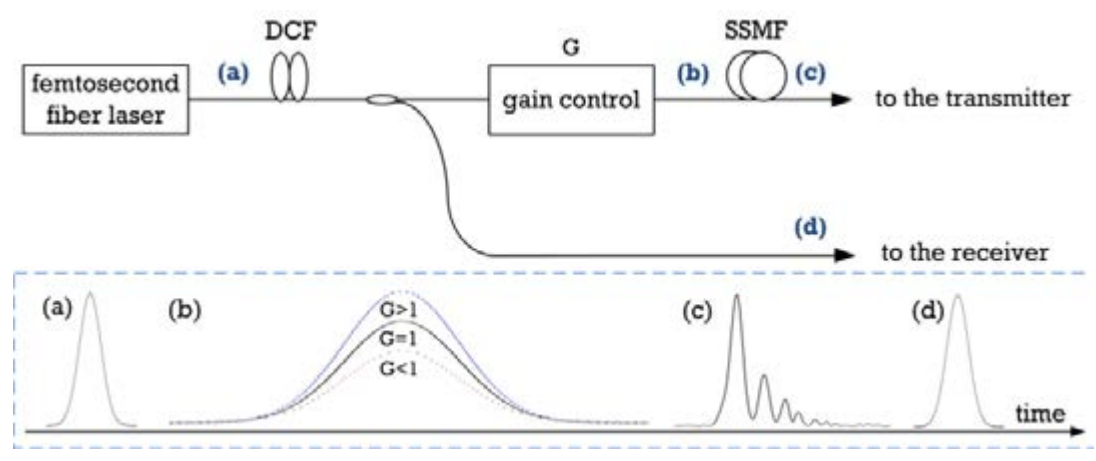

Figure 1: Pulse shaping scheme performed in the fiber distribution link. 


\section{Simulations}

To validate the proposed pulse shaping system the NLSE (4) has been numerically solved using the split-step Fourier method [13]. Numerical simulations have been carried out including SOD, TOD, loss and SPM assuming a 100 fs optical pulse propagating through the scheme shown in Fig. 1. The next table includes the values considered for the different parameters of the numerical simulation

Table 1.- Simulation parameters.

\begin{tabular}{|c|c|c|}
\hline Block & Parameter & Value \\
\hline DCF & $\mathrm{D}$ & $-100 \mathrm{ps} / \mathrm{km} \cdot \mathrm{nm}$ \\
\hline & $\mathrm{S}$ & $0(b)$ and $0.08 \cdot 10^{3} \mathrm{~s} / \mathrm{m}^{3}(c$ to $f)$ \\
\hline & $\mathrm{n}_{2}$ & $2.6 \cdot 10^{-20} \mathrm{~m}^{2} / \mathrm{W}$ \\
\hline & $\alpha$ & $0.5 \mathrm{~dB} / \mathrm{km}$ \\
\hline & Aeff & $50 \mu \mathrm{m}^{2}$ \\
\hline & $\mathrm{n}_{\mathrm{g}}$ & 1.46 \\
\hline Gain control & Length & $6 \mathrm{~m}(b$ to $e), 60 \mathrm{~m}(f)$ \\
\hline SSMF & $\mathrm{G}$ & $16.667 \mathrm{ps} / \mathrm{km} \cdot \mathrm{nm}$ \\
\hline & $\mathrm{D}$ & $0(b)$ and $0.08 \cdot 10^{3} \mathrm{~s} / \mathrm{m}^{3}(c$ to $f)$ \\
\hline & $\mathrm{S}$ & $2.6 \cdot 10^{-20} \mathrm{~m}^{2} / \mathrm{W}$ \\
\hline & $\mathrm{n}_{2}$ & $0.2 \mathrm{~dB} / \mathrm{km}$ \\
\hline & $\alpha$ & $80 \mu \mathrm{m}^{2}$ \\
\hline & Aeff & 1.46 \\
\hline & $\mathrm{n}_{\mathrm{g}}$ & $36 \mathrm{~m}(b$ to $e), 360 \mathrm{~m}(f)$ \\
\hline & Length &
\end{tabular}

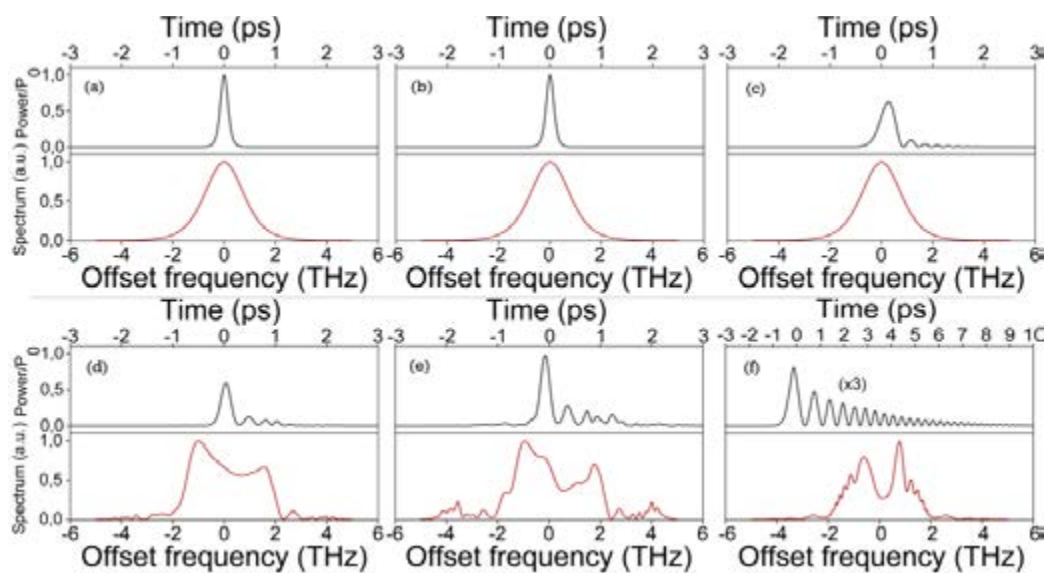

Figure 2: (a)-(f) Shaped pulses and their corresponding spectra for different scenarios 
Due to the splitter and possible splices $6 \mathrm{~dB}$ of power losses are assumed between the DCF and the gain control stage. Results are shown in Fig. 2, where the effect of TOD and SPM is appreciated by changing the amount of fiber (and therefore the accumulated $\beta_{3}$ ), G and the pulse peak power. Fig. 2 (a) shows the initial pulse as well as its spectrum. Fig. 2 (b) and Fig. 2 (c) assume a peak power of $1 \mathrm{~W}$, thus no SPM is generated and their spectra remain unchanged. For the first one the TOD is not considered and therefore the pulse after SOD compensation is the same as the initial one, while for Fig. 2 (c) the optical pulse breaks into a set of sub pulses due to the effect of TOD. A peak power of $630 \mathrm{~W}$, which corresponds to the peak power to be employed in the experimental measurements, is considered from now on. The amplitude of the pulse is increased between the DCF and SSMF sections so SPM arises as it is clearly observed in their spectra. A power gain of 6 and 12 is assumed for Fig. 2 (d) and Fig. 2 (e), respectively. The SPM reinforces the pulse breaking process originated by TOD. By increasing $G$ more pulses are obtained with increasing amplitude. Fig. 2 (f) shows results considering 10 times longer DCF and SSMF. Increasing lengths raise not only the accumulated TOD but also the SPM effect due to the larger pulse-medium interaction time. The time difference between two consecutive pulses is larger since the $\mathrm{THz}$ frequencies generated directly depend on the accumulated TOD. As expected from the theory [12] symmetrical twopeak spectra are obtained as a consequence of the SPM occurring in SOD-compensated fiber links.

\section{Experimental results}

Experiments were carried out according to the setup shown in Fig. 3, where InGaAs-InAlAs PCAs were used in a classical THz-TDS system.

Ultra-short optical pulses (100 fs) with a peak power of $630 \mathrm{~W}$ (average power of $5 \mathrm{dBm}$ at a $50 \mathrm{MHz}$ repetition rate) are dispersion pre-compensated employing DCF. Pre-compensation was performed to avoid unwanted pulse spreading at the input of the PCA employed as receiver while avoiding nonlinearities by reducing the pulse peak power through the optical fiber pigtails. The DCF length was estimated to compensate for the accumulated SOD of the system, 
which was measured by means of an optical network analyzer (Advantest Q7760). This device is able to accurately measure, among other parameters, the length and accumulated dispersion of a fiber link by modulating a tunable continuous wavelength laser with a sweeping frequency and a certain processing of the optical signal before performing photo-detection.

An optical coupler splits these wide pulses to both transmitter and receiver. Optical pulse shaping is carried out in the transmitter part by controlling two different parameters: accumulated TOD and SPM intensity. The former is determined by the length of the SSMF while the latter is varied according to the pump current injected to the Erbium-doped Fiber Amplifier (EDFA). The shaped pulse is applied to an $18 \mathrm{~V}$-biased fiber-pigtailed PCA [5]. This device carries out the photo-detection process and couples most of the energy to free-space by means of a high-resistivity silicon hyper-hemispherical lens. The $\mathrm{THz}$ signal propagates through free-space to the receiver PCA where the terahertz signal is sampled by changing the delay of optical pulses arriving at the receiver. A motorized optical delay line (MODL) was employed to perform these measurements. Phase-sensitive detection with an integration time of $30 \mathrm{~ms}$ using a lock-in amplifier and optically chopping (OC) the amplitude of the $\mathrm{THz}$ signal at $1 \mathrm{kHz}$ was employed to carry out the measurements. Both PCAs were placed at a short distance (a few $\mathrm{cm}$ ) without employing any kind of collimating system for the radiated signal. Therefore we can observe even lowfrequency components (below $100 \mathrm{GHz}$ ) which would be removed through quasi-optic propagation.

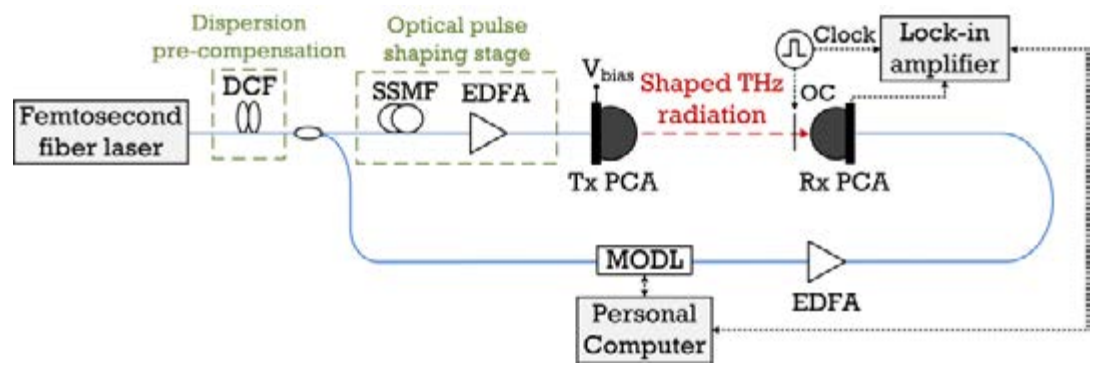

Figure 3: Experimental set-up for generation of shaped $\mathrm{THz}$ radiation. 
After SOD compensation optical pulses with a full-width at half maximum (FWHM) of 200 fs were measured at both PCA inputs for the case of low average optical power $(<10 \mathrm{dBm})$, when optical pulse breaking was negligible (i.e. the system works as a conventional THzTDS system). By increasing the optical power in the fiber of the transmitter path the combined effect of TOD and SPM intensifies and pulse breaking becomes noticeable. This control can be done dynamically, as in our setup by means of the pump current of the EDFA, or it can be fixed by design with a proper combination of laser power and attenuation of fiber splices.

Fig. 4 shows the optical spectra obtained for different pump conditions and a resolution bandwidth of $0.2 \mathrm{~nm}$. The spectrum widening is clearly observed for high EDFA output power values. A certain asymmetry in the spectrum tails is observed due to the gain bandwidth of the EDFA. The spectra with SPM look similar to those obtained in the simulations although differences due to the finite bandwidth of the EDFA gain (not considered in the simulations) are appreciated.

Results obtained for an EDFA pump current of 0.84 A, corresponding to an output power of $13 \mathrm{dBm}$, are shown in Fig. 5. A typical THz-TDS response obtained for an EDFA output power of 9.5 $\mathrm{dBm}$ showing frequency components up to $1 \mathrm{THz}$ is included for comparison purposes. In both cases the EDFA placed at the input of the receiver PCA provided an average power of $9 \mathrm{dBm}$ with no pulse breaking. Narrowband $\mathrm{THz}$ radiation centered at $500 \mathrm{GHz}$ with a FWHM close to $200 \mathrm{GHz}$ was obtained. As it can be observed a noticeable amount of radiation is obtained around the DC component. The proposed architecture can be considered to apply a finite impulse response (FIR) filter response [14] to the input femtosecond pulse. Therefore low-frequency components are expected to appear as well as radiation periodicity. The first one can be appreciated in Fig. 5, where some radiation appears around $100 \mathrm{GHz}$. Due to the non-flat response of the radiation emitted by a PCA, as well as the relatively high central frequency of the shaped radiation, the periodicity (at multiples of the repetition frequency of the train of pulses $n \cdot 500 \mathrm{GHz}$ ) is not observed here. 


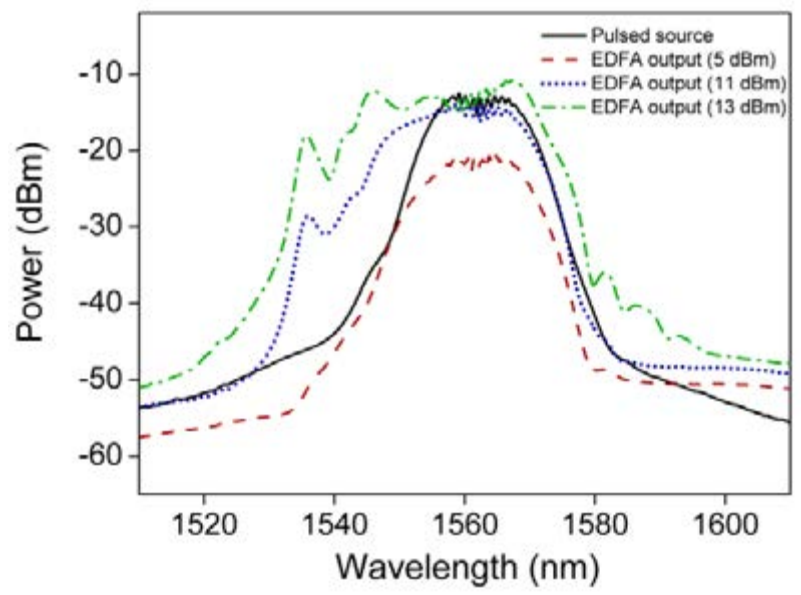

Figure 4: Optical spectra of the source and at the output of the EDFA for different pump conditions.
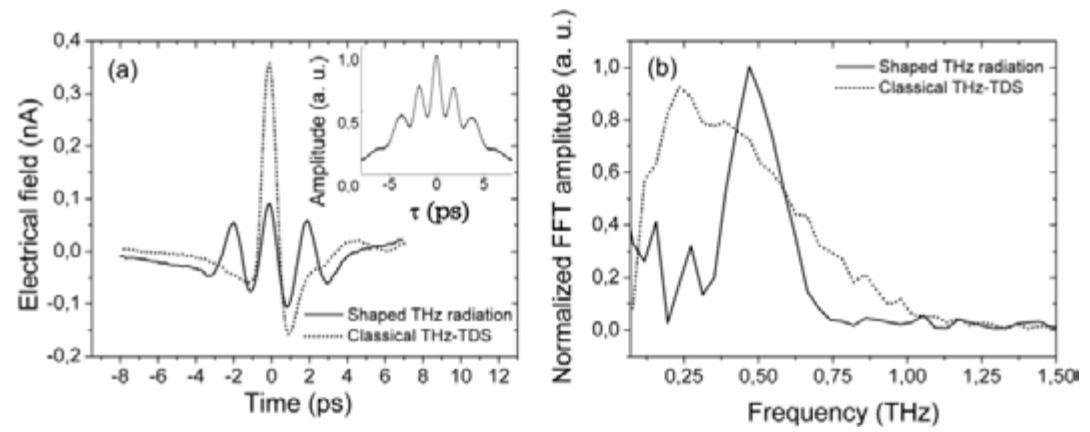

Figure 5: (a) Time-domain $\mathrm{THz}$ waveforms with (continuous) and without (dashed) pulse shaping. Inset: optical autocorrelation trace of the optical pulse employed to perform pulse shaping. (b) Spectra of narrowband $\mathrm{THz}$ radiation (continuous line) and a conventional THz-TDS system without pulse shaping (dashed).

For demonstrating the tunability of the system, accumulated TOD in the transmitter branch was varied while the EDFA pump current was kept constant. In this part of the experiment the ultra-short optical pulse broke in two pulses. Experiments were carried out for different TOD values and it was observed that by removing (adding) fiber, which corresponds to lower (higher) accumulated TOD values, the central frequency of the $\mathrm{THz}$ radiation could be increased (decreased). Even when adding or removing fiber widens the optical 
pulse due to SOD, as long as the accumulated $\beta_{2}$ remains low the nonlinear effects will dominate and the shaping mechanism will still be valid. Since the repetition rate of the femtosecond fiber laser is 50 $\mathrm{MHz}$ the time difference between consecutive pulses is 20 ns. According to the $5 \mathrm{~ns} / \mathrm{m}$ delay of the optical fiber, sections whose length in meters is an integer multiple of 4 will be added or removed in order to measure the $\mathrm{THz}$ pulse with the current alignment.

Fig. 6 shows the experimental results, where time domain $\mathrm{THz}$ traces as well as its corresponding spectra are included. Results were compared to the response of a two-tap FIR filter taking into account the spectral response of the THz-TDS system (Fig. 5). Central frequencies of $350 \mathrm{GHz}, 525 \mathrm{GHz}$ and $800 \mathrm{GHz}$ were obtained, showing the tunability of the proposed pulse shaper. The $350 \mathrm{GHz}$ spectral response also includes a replica at $700 \mathrm{GHz}$ due to the periodicity of the implemented filter, which could not be observed for higher central frequencies due to the limited bandwidth of the THz-TDS system.

The proposed system allows not only tunability but also dynamic reconfigurability of the bandwidth of the generated $\mathrm{THz}$ narrowband signal. It is achieved by controlling the number of pulses in which the original optical pulse breaks up. The intensity of the SPM effect can be controlled by means of the EDFA gain. Measurements were carried out for a central frequency of $600 \mathrm{GHz}$. EDFA output power values were 11.5, 13 and $15 \mathrm{dBm}$. For this set of powers 2, 3 and 4 pulses were obtained, respectively. Results included in Fig. 7 show how by increasing the number of pulses the $\mathrm{THz}$ radiation becomes narrower. The FWHM of the signals decreased up to approximately $150 \mathrm{GHz}$.
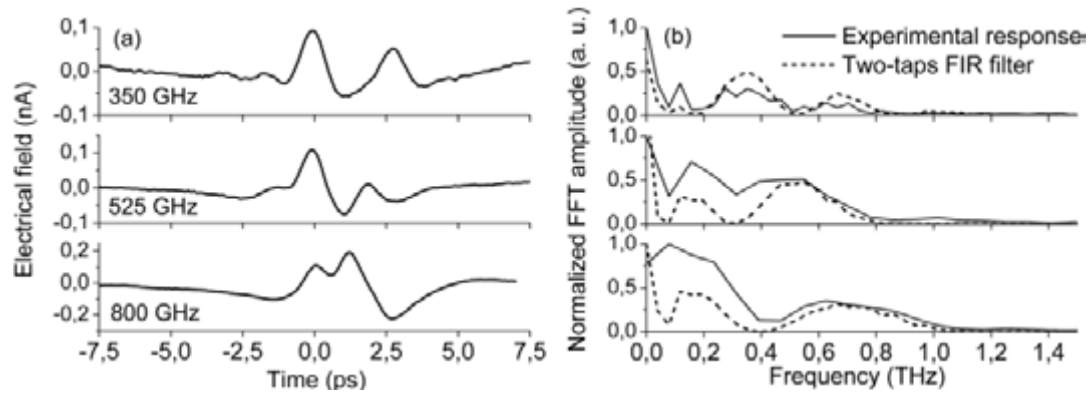

Figure 6: (a) Time-domain $\mathrm{THz}$ waveforms and (b) their corresponding spectra for different values of accumulated TOD. 

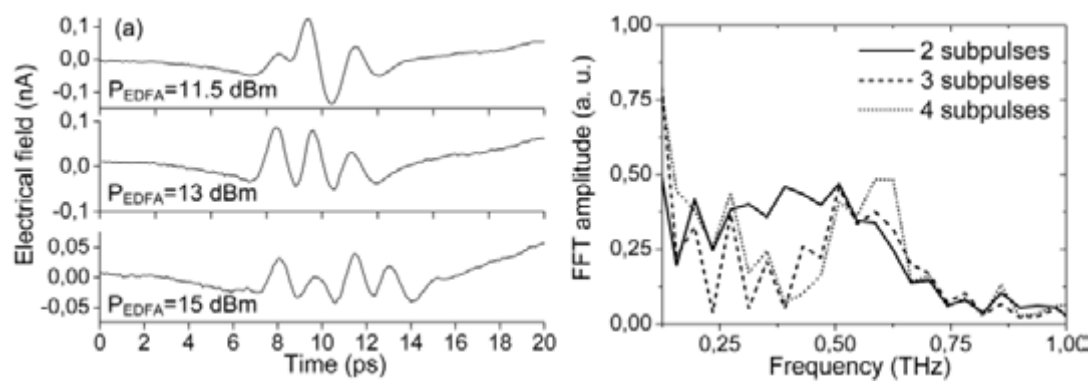

Figure 7: (a) Time-domain $\mathrm{THz}$ waveforms and (b) their corresponding spectra for different pump currents applied to the EDFA.

Although the pump current of an EDFA and the amount of SSMF have been changed in order to demonstrate the flexibility of the system, radiation tuning could be carried out in different ways. The effective amount of TOD could be dynamically tuned by employing a phase modulator since this device has already been employed in active dispersion compensation [15]. And the optical power could be controlled through the pump current of the femtosecond laser as long as it doesn't affect the performance of the ultra-short pulse. In this case no additional devices could be required in order to perform the radiation shaping.

\section{Conclusions}

An all-fiber system for the generation of narrowband $\mathrm{THz}$ radiation based on the breaking of ultra-short optical pulses by means of the effect of third-order dispersion and its enhancement due to selfphase modulation is proposed. The proposed scheme allows for both tunability and reconfigurability. The technique shows potential to be implemented straightforwardly in fiber-based THz-TDS systems without the need of additional components, improving the flexibility of these systems. Numerical simulations as well as experimental measurements showing the principle of operation have been presented. Narrowband $\mathrm{THz}$ radiation is obtained along the full $\mathrm{THz}$ system bandwidth, generating radiation centered up to $800 \mathrm{GHz}$ with fullwidth at half maximum values from $150 \mathrm{GHz}$. 


\section{References}

[1] P.H. Siegel, "Terahertz technology," IEEE Trans. Microwave Theory Tech., vol. 50, no. 3, pp. 910-928, 2002.

[2] M. Tonouchi, "Cutting-edge terahertz technology," Nature Photon. vol. 1, pp. 97-105, 2007.

[3] J. Faist, F. Capasso, D.-L. Sivco, C. Sirtori, A.-L. Hutchinson, and A.-Y- Cho, "Quantum cascade laser," Science, vol. 264, no. 5158, pp. 553-556, April 1994.

[4] D. Saeedkia and S. Safavi-Naeini, "Terahertz photonics: optoelectronic techniques for generation and detection of terahertz waves," J. Lightwave Technol., vol. 26, no. 15, pp. 2409-2423, Aug. 2008.

[5] B. Sartorius, H. Roehle, H. Künzel, J. Böttcher, M. Schlak, D. Stanze, H. Venghaus, M. Schell, "All-fiber terahertz time-domain spectrometer operating at $1.5 \mathrm{\mu m}$ telecom wavelengths," Opt. Express, vol. 16, no. 13, pp. 9565-9570, Jun. 2008.

[6] A.S. Weling, T.F. Heinz, "Enhancement in the spectral irradiance of photoconducting terahertz emitters by chirped-pulse mixing," J. Opt. Soc. Am. B, vol. 16, no. 9, pp. 1455-1467, 1999.

[7] O. Levinson, M. Horowitz, "Generation of complex microwave and millimeter-wave pulses using dispersion and Kerr effect in optical fiber systems," J. Lightwave Technol., vol. 21, no. 5, pp. 1179-1187, May 2003.

[8] J. Stigwall and A. Wiberg, "Tunable terahertz signal generation by chirped pulse photomixing," IEEE Photon. Technol. Lett., vol. 19, no. 12, pp.931-933, 2007.

[9] S. Vidal, J. Degert, J. Oberlé, and E. Freysz, "Femtosecond optical pulse shaping for tunable terahertz pulse gen

[10] eration," J. Opt. Soc. Am. B, vol. 27, no. 5, pp.1044-1050, 2010.

[11] G. P. Agrawal, Nonlinear fiber optics, 3rd edn., San Diego, CA: Academic, 2001, p.1

[12] G. P. Agrawal, Nonlinear fiber optics, 3rd edn., San Diego, CA: Academic, 2001, pp.49

[13] G. P. Agrawal, Nonlinear fiber optics, 3rd edn., San Diego, CA: Academic, 2001, p.97 
[14] G. P. Agrawal, Nonlinear fiber optics, 3rd edn., San Diego, CA: Academic, 2001, pp.51-55

[15] J. Capmany, B. Ortega, and D. Pastor, "A tutorial on microwave photonic filters," J. Lightwave Technol., vol. 24, no. 1, pp. 201-229, Jan. 2006.

[16] E. Hellström, H. Sunnerud, M. Westlund, and M. Karlsson, "Thirdorder dispersion compensation using a phase modulator," J. Lightwave Technol., vol. 21, no. 5, pp. 1188-1197, May 2003. 


\title{
Paper E
}

"Terahertz radiation shaping based on optical spectrum modulation in the time domain"

\author{
J. Palací, A. Bockelt and B. Vidal
}

Opt. Express, Vol. 20, No. 21, pp. 23117-23125, September 2012 



\title{
Terahertz radiation shaping based on optical spectrum modulation in the time domain
}

\author{
Jesús Palací, Alexander Bockelt and Borja Vidal \\ Nanophotonics Technology Center, Universidad \\ Politécnica de Valencia, 46022 Valencia, Spain
}

\begin{abstract}
A terahertz shaping system based on optical fiber components as opposed to traditional free-space solutions is proposed. It is based on the time-domain modulation of the optical source spectrum. Standard single-mode fiber distributes and disperses the pulse before filtering its spectral components by means of the cross-gain and cross-phase modulation effects taking place in an interferometric semiconductor optical amplifier structure. Experimental measurements are obtained, showing the tunability of the system as well as its reconfigurability.
\end{abstract}

\section{Introduction}

Submillimeter radiation shows a great potential in a wide range of fields from imaging and material identification to biology and communications [1]. However the generation and detection in this frequency band remains difficult. Electronic commercial solutions are available up to a few hundreds of $\mathrm{GHz}$ while quantum-cascade lasers (QCLs) [2] are suitable for frequencies over $1 \mathrm{THz}$ although cryogenic operation prevents wide application.

Photonic schemes require an optical source but they are easy to operate, more flexible and able to cover most of the $\mathrm{THz}$ gap. Among the different photonic solutions, such as periodically poled lithium niobate (PPLN) [3], electro-optic sampling using different crystals [4] or Cherenkov radiation [5], photoconductive antennas (PCAs) [6] are widely employed. PCAs, usually fed by Ti:Sapphire lasers, are key components in $\mathrm{THz}$ time-domain spectroscopy (THz-TDS) systems. Recently new commercial PCA designs with lower energy gaps that can be excited with $1.5 \mu \mathrm{m}$ light have reached the market [7]. Therefore cheaper optical sources developed for the telecommunication industry can be employed as well as other devices like Erbium-doped fiber amplifiers (EDFAs), electro-optic modulators (EOMs), 
semiconductor optical amplifiers (SOAs) and single-mode and specialty optical fibers for signal distribution and processing.

The processing of ultra-short optical pulses has been extensively studied due to its applicability in different areas [8]. Most of the proposed solutions are based on the direct control of the optical spectrum of the pulses. Typically, the different components are spatially separated using diffractive elements as gratings, before a phase/amplitude spatial modulation is applied on the different spectral components by means of phase masks [9], reconfigurable metallic structures [10] or spatial light modulators [11]. The latter provides much more flexibility enabling the implementation of quasiarbitrary waveforms. However free-space components are susceptible of misalignment, long-term stability problems and result in bulky systems. On the other hand, the recent increase in the performance of $\mathrm{THz}$ sources excited with $1.5 \mu \mathrm{m}$ sources $[12,13]$ motivates research for fiber-based processing of ultra-short pulses with applicability to the $\mathrm{THz}$ band. Recently, a technique based on the optical heterodyning of a time-stretched ultra-short optical pulse with its delayed replica to generate narrow-band $\mathrm{THz}$ radiation [14] has been demonstrated using fiber-based components [15]. Despite of its good performance and simplicity, the bandwidth of the generated radiation is related to its central frequency, limiting the flexibility of the system. Another approach which has been applied to the microwave range relies on the time-domain modulation of the optical spectrum, obtaining high timebandwidth products and providing accurate control over the generated waveforms [16]. In this architecture optical fiber is employed as the dispersive medium and EOMs shape the time-decomposed spectrum. Thus the performance of the setup is limited by the stretched pulse duration and the modulator speed, which rarely exceeds a few tens of $\mathrm{GHz}$.

SOAs have been traditionally employed for applications requiring short time responses like optical switching [17]. Particularly, multiple quantum well (MQW) optical amplifiers show short-term amplitude and phase dynamics below the ps [18], which makes them suitable for most ultrafast applications.

The generation of certain terahertz waveforms to concentrate the radiation into a given frequency band locally raises the energy density and therefore increases the dynamic range of the system at those 
frequencies [19]. In this work an all-fiber all-optical dynamicallycontrolled THz pulse shaper is proposed and demonstrated. It is based on processing the optical source in a SOA Mach-Zehnder interferometer (SOA-MZI). The different spectral components are shaped in the time domain employing the cross-gain (XGM) and crossphase modulation (XPM) effects taking place in the SOA-MZI. Experimental measurements have been performed validating the feasibility of the technique as well as the tunability of the $\mathrm{THz}$ central frequency and its bandwidth.

\section{Principle of operation}

The proposed technique is based on shaping the spectrum of a pulsed source by changing the interference conditions of an SOA-MZI in which the time-stretched pulses have been injected. Thanks to the fast response of this structure it is possible to separately modulate the different spectral components.

Figure 1(a) shows the schematic of the SOA-MZI as well as the interference experienced by an optical pulse entering the structure. The optical pulse is injected into both SOAs by means of several integrated optical couplers. Pulses in both branches of the interferometer are amplified by their respective SOA experiencing amplitude and spectral phase changes according to the bias current injected to the amplifiers. The output stage is symmetric with respect to the input and combines the pulses. Constructive or destructive interference occurs for the phase conditions shown.

Consider the scenario of Fig. 1(b). An optical pulse to be shaped is linearly chirped and injected into the SOA-MZI. An optical waveform used to control the shaping of the previous pulse counter-propagates through the upper SOA. Assuming that the shaping waveform is a single optical pulse with a duration much shorter than the recovery time of SOA1, $\tau_{\text {rec }}$, and that the gain response of SOA1 follows a negative exponential as in [20], the electrical fields at the output of the SOAs are given by

$$
\begin{gathered}
E_{S O A_{1}}^{\text {out }}(t)=\sqrt{I_{S O A_{1}}\left(1-\alpha_{X G M} \cdot \exp \left(-t / \tau_{\text {rec }}\right)\right)} \exp \left(j\left(\varphi_{S O A_{1}}+\varphi_{X P M} \cdot \exp \left(-t / \tau_{\text {rec }}\right)\right)\right) \\
E_{S O A_{2}}^{\text {out }}(t)=\sqrt{I_{S O A_{2}}} \exp \left(j \varphi_{S O A_{2}}\right) \exp (j \pi)
\end{gathered}
$$




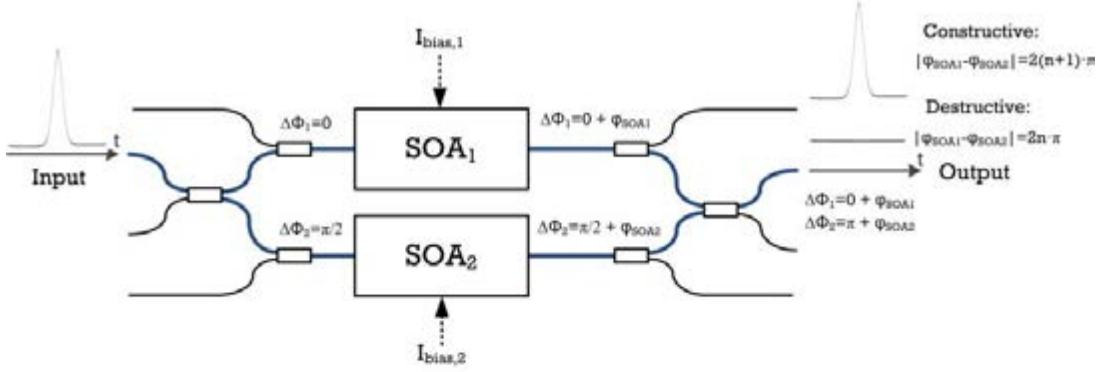

(a)

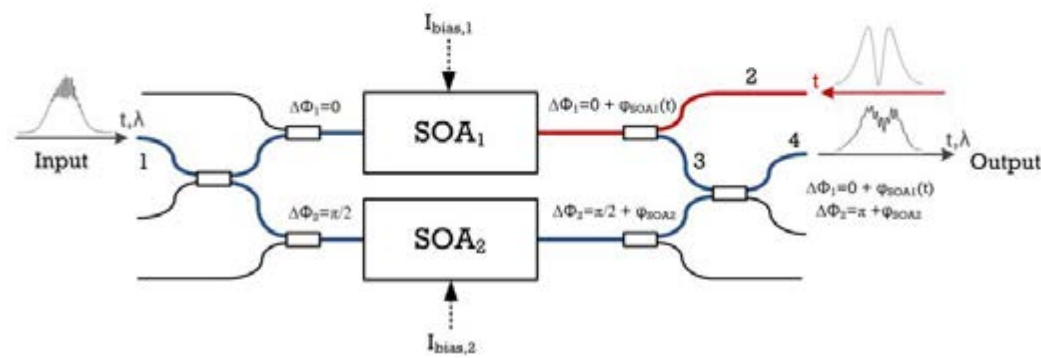

(b)

Fig. 1. (a) SOA interferometer block diagram. (b) SOA interferometer considering a chirped pulse and an additional counter-propagating pulse.

being $\mathrm{I}_{\mathrm{SOA} 1}$ and $\mathrm{I}_{\mathrm{SOA} 2}$ the average power at the output of the SOAs, $\alpha_{\mathrm{XGM}}$ a factor that measures $\mathrm{SOA}_{1}$ gain reduction due to gain saturation when the short pulse arrives, $\varphi_{\mathrm{SOA} 1}$ and $\varphi_{\mathrm{SOA} 2}$ the phase shifts introduced by the SOAs onto the chirped pulse and $\varphi_{\mathrm{XPM}}$ the maximum phase shift originated by XPM. The last exponential of Eq. (2) is for the $\pi$ phase shift introduced by the optical couplers in $\mathrm{SOA}_{2}$ with respect to $\mathrm{SOA}_{1}$. The electrical fields combine by means of the output couplers of the MZI structure and an optical power at the output of the system proportional to $\left|\mathrm{E}^{\text {out }}{ }_{\mathrm{SOA} 1}(\mathrm{t})+\mathrm{E}_{\mathrm{SOA} 2}^{\text {out }}(\mathrm{t})\right|^{2}$ is obtained.

In the initial state, $\mathrm{t}<0$, no external pulse is injected into the system. Assuming identical SOAs, when the same biasing currents $\mathrm{I}_{\text {bias }}$ are applied to both devices $\mathrm{I}_{\mathrm{SOA} 1}$ equals $\mathrm{I}_{\mathrm{SOA} 2}$ as well as $\varphi_{\mathrm{SOA} 1}$ with respect to $\varphi_{\mathrm{SOA} 2}$. Thus, the chirped pulse is suppressed. However, the arrival at $\mathrm{t}=0$ of a pulse with enough energy to reduce the carrier density of $\mathrm{SOA}_{1}$ will temporarily change its gain and phase profiles 
through the $\alpha_{\mathrm{XGM}}$ and $\varphi_{\mathrm{XPM}}$ parameters, putting the system out of the destructive state and impressing variations onto the chirped pulse (i.e. performing an all-optical pulse shaper at the output of port 4). The transform-limited version of the shaped spectrum is obtained after compensating for the initial chirp.

\section{Experimental results}

Figure 2 shows the THz-TDS architecture. Gaussian-shaped optical pulses are generated in a femtosecond fiber-laser (fs-FL) with an average power of $5 \mathrm{dBm}$ at a $50 \mathrm{MHz}$ repetition rate and an estimated full-width at half maximum (FWHM) duration of 100 fs. These pulses are time-stretched by means of $4.16 \mathrm{~km}$ of dispersion compensation fiber (DCF) with a dispersion parameter (D) of $-100 \mathrm{ps} / \mathrm{km} \cdot \mathrm{nm}$ and a dispersion slope $(\mathrm{S})$ of $0.08 \mathrm{ps} / \mathrm{km} \cdot \mathrm{nm}^{2}$. Thus, the pulses suffer a total dispersion $\left(\mathrm{D}_{\mathrm{T}}\right)$ of $-416 \mathrm{ps} / \mathrm{nm}$ with a slope of $0.33 \mathrm{ps} / \mathrm{nm}^{2}$. By using DCF as the dispersive medium, with a much higher dispersion parameter to that of standard single-mode fiber (SSMF) and a similar dispersion slope, the influence of the dispersion slope on the system performance becomes low enough to be neglected.

A polarization controller (PC) is included at the input of the SOA-MZI to account for the polarization dependence of the interferometric structure. The SOA-MZI, manufactured by CIP, comprises two identical bulk SOAs and a set of optical couplers with a $3 \mathrm{~dB}$ splitting ratio to form an interferometer. A transmission-line model technique was applied to model the SOAs according to [21]. The length of both SOAs is $500 \mu \mathrm{m}$ with a linewidth enhancement factor of 8 , a noise figure of $8 \mathrm{~dB}$, a confinement factor of 0.3 and a spontaneous emission factor of 1.5. The transparency current is estimated to be $17 \mathrm{~mA}$ and the unsaturated fiber-to-fiber gain is 13 dB. Carrier lifetimes of 1 ns and 300 ps were measured under 100 and $250 \mathrm{~mA}$ biasing, respectively.

The optical waveform used to control the SOA-MZI (shaping waveform) is derived from the same source than the pulses to be shaped. In this case a fiber Mach-Zehnder interferometer (MZI) was implemented using an optical delay line (ODL) to introduce a delay difference between its arms of $\tau_{\text {ODL }}$ and select two spectral bands of the chirped pulse. SSMF with a length of $\mathrm{L}$ was included for pulse widening. Since two bands are filtered a train of pulses with repetition 
frequency $f_{\text {rep }}$ is obtained after compensation for the initial chirp. Its value is related to the different parameters of the system as follows.

$$
f_{\text {rep }} \approx \frac{\tau_{O D L}}{\left|D_{T}\right|}
$$

The effect of the dispersion slope has been neglected due to its low influence. Regarding the temporal response of the SOA-MZI structure, for shaping waveforms much shorter than the gain recovery time of the SOAs the bandwidth of the filtered bands is given by the recovery time of the SOA while for longer ones the filtered bands approximately follow the shaping waveform. From now on the variable $\tau_{\text {system }}$ is used for the FWHM duration of the system response to an arbitrary shaping pulse width. The FWHM bandwidth of the filtered spectrum $\Delta v$ can be expressed as

$$
\Delta v \approx \frac{\tau_{\text {system }}}{\left|D_{T}\right|}
$$

The FWHM duration of the pulse train envelope can be expressed in terms of the time-bandwidth product (TBP) and Eq. (4) as

$$
\Delta \tau \approx \frac{T B P\left|D_{T}\right|}{\tau_{\text {system }}}
$$

According to Eqs. (3-5) and the different parameters of our system, a train of pulses with a repetition frequency of $0.3 \tau_{\mathrm{ODL}}(\mathrm{ps})$ $\mathrm{GHz}$ and an approximated envelope duration of $1.67 / \tau_{\text {system }}(\mathrm{ps})$ ns for a TBP of 0.5 is obtained at the output of the SOA-MZI structure. The bandwidth of the generated $\mathrm{THz}$ radiation is expected to follow $0.3 \tau_{\text {system }}(\mathrm{ps}) \mathrm{GHz}$.

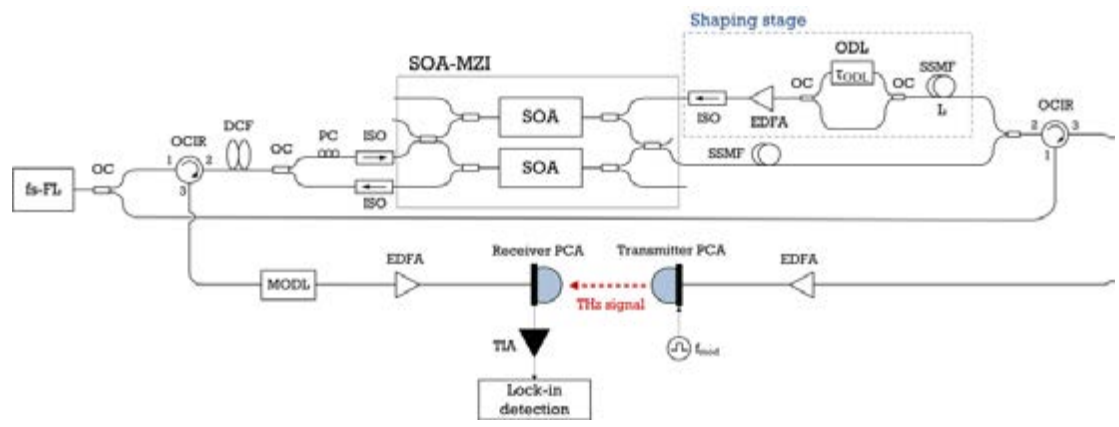

Fig. 2. Optical pulse shaper integrated in a fiber-based THz-TDS system. 


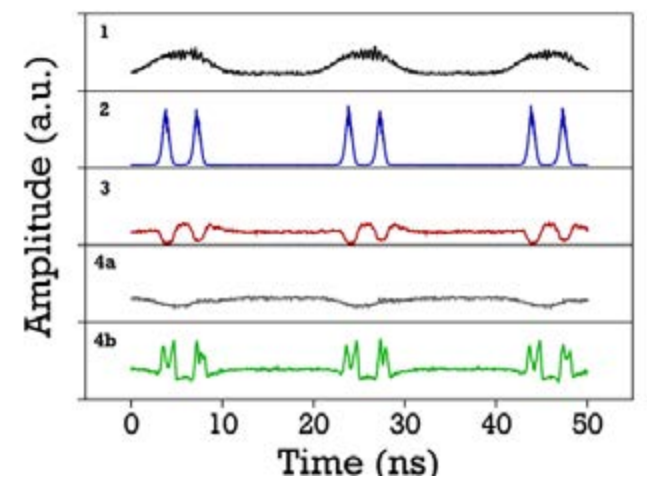

Fig. 3. Photo-detection traces at different points of the SOA-MZI structure as shown in Fig. 1(b).

The traces included in Fig. 3 were obtained in a digital communication analyzer with $30 \mathrm{GHz}$ bandwidth and correspond to the points shown in Fig. 1(b). Trace 1 is for the chirped pulse at the input of the SOA-MZI while trace 2 is the shaping waveform. Trace 3 shows the XGM effect induced by the carrier saturation arising from the shaping waveform injection in the upper SOA. Traces $4 \mathrm{a}$ and $4 \mathrm{~b}$ are for the SOA-MZI output without and with the shaping signal being injected, respectively. For the latter, the combined effect of XGM and XPM can be seen [20]. A slight asymmetry in the biasing currents was observed to provide a better extinction ratio for the spectral filtering. Indeed, the optimum SOAs biasing currents were found to be 110 and $100 \mathrm{~mA}$ for the upper and lower, respectively.

After the SOA-MZI the pulse is dispersion compensated by means of $25 \mathrm{~km}$ of SSMF with a dispersion parameter of $16.7 \mathrm{ps} / \mathrm{km} \cdot \mathrm{nm}$. Due to the need of time alignment between the transmitter and receiver branches of the THz-TDS system, compensating for possible sources of error in both branches turns out to be important. We experimentally observed how the initial difference in fiber length between the transmitter and receiver branches led to time fluctuations of the $\mathrm{THz}$ pulse in the order of hundreds of ps. This occurs because of fiber expansion and contraction due to temperature changes. To solve this problem the optical pulses of both transmitter and receiver pulse must travel similar distances. This is done by the use of optical couplers (OC), optical circulators (OCIR) and isolators (ISO). The 
1x2 OCs employed in the system had a coupling ratio of 50/50 and a directivity of $50 \mathrm{~dB}$. The insertion losses of the OCs, ISOs and OCIRs were $0.5,0.7$ and $1 \mathrm{~dB}$, respectively. The ISOs and the OCIRs provided isolation larger than $40 \mathrm{~dB}$. The OCIRs showed both directivity (signal from port 1 to 3 ) and return loss higher than $50 \mathrm{~dB}$. According to these values both signal paths could be considered independent as confirmed by the experimental measurements.

A motorized optical delay line (MODL) is employed to sweep the $\mathrm{THz}$ traces in $100 \mathrm{fs}$ steps and EDFAs to boost the optical signals into the $\mathrm{mW}$ range before feeding the $1.5 \mu \mathrm{m}$ PCAs. Lock-in detection after $10^{6} \mathrm{~V} / \mathrm{A}$ of trans-impedance amplification (TIA) is employed to record the electric field. The transmitter antenna is square biased from -10 to $+10 \mathrm{~V}$ at a frequency $\left(\mathrm{f}_{\text {mod }}\right)$ of $1 \mathrm{kHz}$.

Before injecting the shaped pulse into the $\mathrm{THz}$ system, autocorrelation traces are obtained. The central frequency and its bandwidth are controlled by means of the MZI delay and the fiber length, respectively. In Fig. 4(a) the duration of the envelope does not change because the fiber length is kept constant and the pulse train duration is directly related to the bandwidth of the filtered spectral bands. Trains of pulses with a repetition period of 2.7, 1.64 and 1.05 ps are obtained for increasing values of $\tau_{\mathrm{ODL}}$ according to Eq. (3). In Fig. 4(b) a set of different values for $\mathrm{L}$ is considered for the same $\tau_{\mathrm{ODL}}$. Fiber reels of 1, 2 and $5 \mathrm{~km}$ widened the optical pulses up to $200 \mathrm{ps}$, $400 \mathrm{ps}$ and $1 \mathrm{~ns}$, respectively, and biasing currents of $300 \mathrm{~mA}$ were used for the SOAs.

For comparison purposes, the THz-TDS system of Fig. 2 is tested without applying any shaping to the signal (by switching off the upper SOA). The estimated FWHM of the pulses applied to both PCAs is $300 \mathrm{fs}$, with an average power of $10 \mathrm{dBm}$ and $5 \mathrm{dBm}$ in the transmitter and receiver, respectively. Recovery of the original pulse duration was not possible since only the second-order dispersion was compensated. Figure 5 shows the $\mathrm{THz}$ trace recorded as well as its Fourier transform.

Finally, the narrowband THz-TDS system is tested in different frequency bands. Figure 6 shows the different $\mathrm{THz}$ waveforms and their corresponding spectra. In Fig. 6(a), as well as in Fig. 5(a), a low frequency component appears as an increasing current slope after the pulse train which is thought to originate from impedance mismatching 
in the antenna. In Fig. 6(b) narrowband radiation with central frequencies of 300,550 and $700 \mathrm{GHz}$ can be seen.

(a)

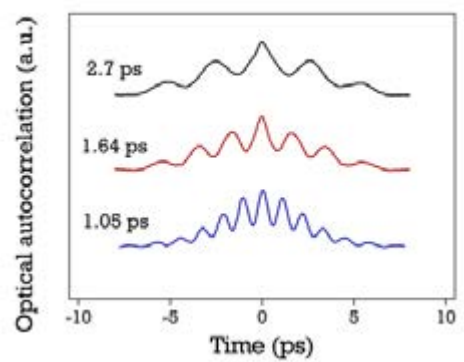

(b)

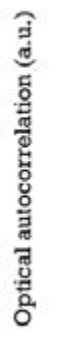

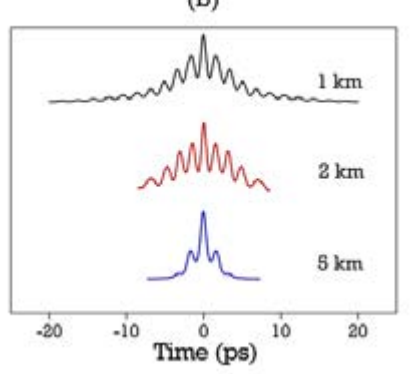

Fig. 4. Optical autocorrelation traces for several values of (a) delay and (b) fiber length in the shaping stage.

(a)

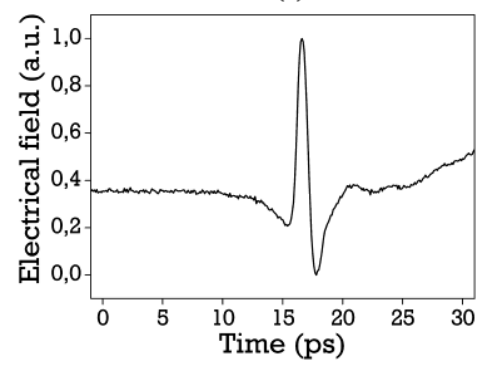

(b)

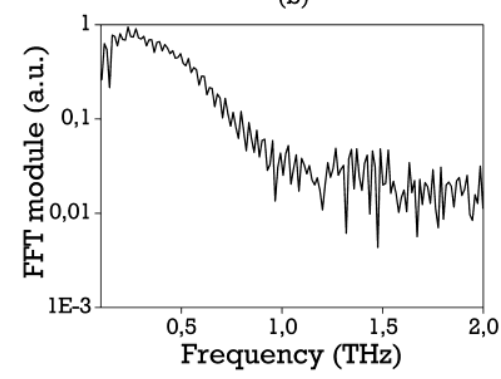

Fig. 5. (a) Time-domain $\mathrm{THz}$ waveform and (b) its corresponding spectra when no shaping is performed.
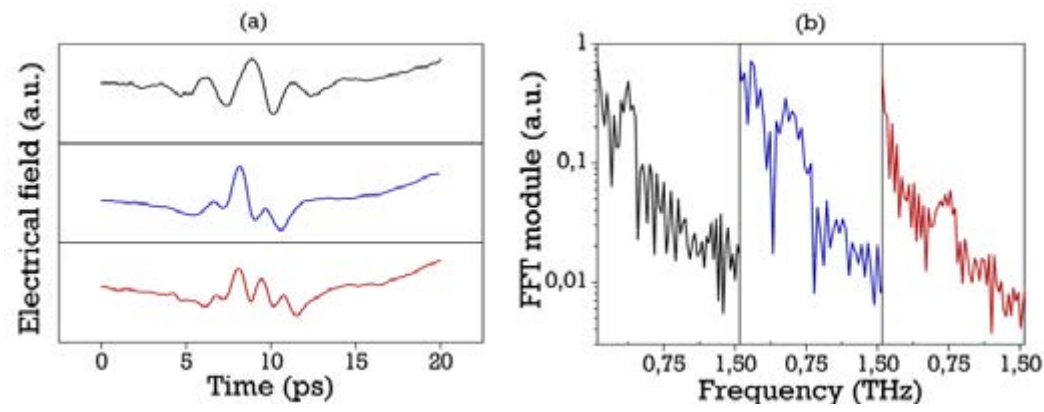

Fig. 6. (a) Time-domain $\mathrm{THz}$ waveforms and (b) their corresponding spectra for different frequency bands. 
(a)

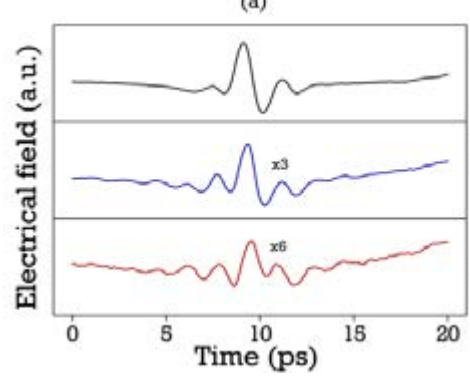

(b)

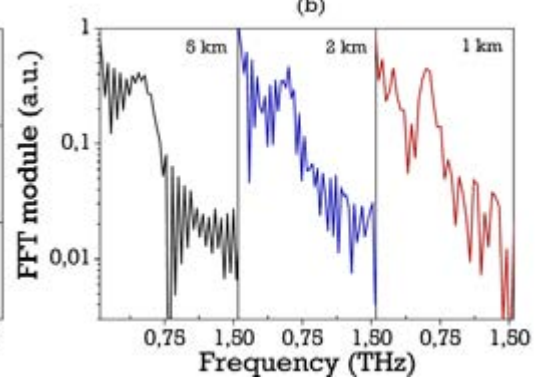

Fig. 7. (a) Time-domain $\mathrm{THz}$ waveforms and (b) their corresponding spectra for bandwidth reconfiguration.

After showing the tunability of the system the $\mathrm{THz}$ radiation bandwidth is controlled by changing the amount of fiber in the shaping stage, $\mathrm{L}$. THz waveforms as well as their spectra are included in Fig. 7 where $\tau_{\mathrm{ODL}}$ is kept constant and the narrowing of the spectrum as the fiber length decreases can be appreciated. Lowfrequency components arising from beating among components of the same spectral band are reduced for narrower $\mathrm{THz}$ bandwidths as shown in Fig. 7. Optical switches can be employed to select among different fiber reels. In this case the amount of fiber in the shaping stage is used to control the width of the $\mathrm{THz}$ radiation, although other approaches could be considered. Phase modulation has been reported to be able to compensate for the dispersion of a fiber link [21]. Therefore it could be employed to dynamically change the radiation bandwidth by increasing or reducing the accumulated dispersion. The SOA dynamics could also be controlled through its bias current or by adding a low wavelength component which would change the response time of the SOA and therefore the selected bands' bandwidth [20].

\section{Conclusions}

An all-optical fiber based $\mathrm{THz}$ pulse shaping scheme based on the optical modulation of the spectrum of ultra-short pulses is proposed and demonstrated. It allows for both tunability and reconfigurability of the emitted radiation through independent system parameters as opposed to previous solutions. Additionally, since it is an all-fiber approach, compact and robust instruments can be implemented. The precision of the modulation of the pulse spectrum is given by the 
recovery time of the SOA and the amount of dispersion that can be introduced onto the signal. High performance could be obtained through a large linear chirping of the signal and fast SOAs, although the introduction of non-linear dispersion could make the generation process more difficult due to the effect of the non-linear mapping of the spectrum in the time domain. The experimental measurements performed agree well with the theoretical analysis performed and prove the feasibility of dynamic $\mathrm{THz}$ processing systems able to concentrate the radiated energy in the spectral region of interest. Although the measurements performed show simple pulse shapes the proposed technique could provide arbitrary pulse shaping by using state-of-the-art SOAs.

\section{References}

[1] P.H. Siegel, "Terahertz technology," IEEE Trans. Microwave Theory Tech., vol. 50, no. 3, pp. 910-928, 2002.

[2] J. Faist, F. Capasso, D.-L. Sivco, C. Sirtori, A.-L. Hutchinson, and A.-Y- Cho, "Quantum cascade laser," Science, vol. 264, no. 5158, pp. 553-556, April 1994.

[3] Y.-S. Lee, T. Meade, V. Perlin, H. Winful and T. B. Norris, "Generation of narrow-band terahertz radiation via optical rectification of femtosecond pulses in periodically poled lithium niobate," Appl. Phys. Lett., vol. 76, pp. 2505-2507, 2000.

[4] Q. Wu and X.- C. Zhang, "Free-space electro-optic sampling of terahertz beams," Appl. Phys. Lett., vol. 67, pp. 3523-3525, 1995.

[5] D. H. Auston, K. P. Cheung, J. A. Valdmanis and D. A. Kleinman, "Cherenkov radiation from femtosecond optical pulses in electro-optic media," Phys. Rev. Lett., vol. 16, pp. 1555-1558, 1984.

[6] D. H. Auston, K. P. Cheung and P. R. Smith, "Picosecond photoconducting Hertzian dipoles," Appl. Phys. Lett., vol. 45, pp. 284286, 1984.

[7] N. Chimot, J. Mangeney, L. Joulaud, P. Crozat, H. Bernas, K. Blary and J. F. Lampin, "Terahertz radiation from heavy-ion-irradiated In0.53Ga0.47As photoconductive antenna excited at $1.55 \mu \mathrm{m}, "$ Appl. Phys. Lett., vol. 87, 193510, 2005. 
[8] A. M. Weiner, "Ultrafast optical pulse shaping: a tutorial review," Opt. Comm., vol. 284, pp. 3669-3692, 2011.

[9] Y. Liu, S.-G. Park and A. M. Weiner, "Terahertz waveform synthesis via optical pulse shaping," IEEE J. Sel. Top. Quantum Electron., vol. 2, pp. 709-719, 1996.

[10] J. Y. Sohn, Y. H. Ahn, D. J. Park, E. Oh and D. S. Kim, "Tunable terahertz generation using femtosecond pulse shaping," Appl. Phys. Lett., vol. 81, pp. 13-15, 2002.

[11] A. M. Weiner, "Femtosecond pulse shaping using space modulators," Rev. Sci. Instrum., vol. 71, pp. 1929-1960, 2000.

[12] R. J. B. Dietz, M. Gerhard, D. Stanze, M. Koch, B. Sartorius and M. Schell, "THz generation at $1.55 \mu \mathrm{m}$ excitation: six-fold increase in $\mathrm{THz}$ conversion efficiency by separated photoconductive and trapping regions," Opt. Express, vol. 19, pp. 25911-25917, 2011.

[13] J. R. Middendorf and E. R. Brown, "THz generation using extrinsic photoconductivity at $1550 \mathrm{~nm}$, " Opt. Express, vol. 20, pp. 16504-16509, 2012 .

[14] A. S. Weling and D. H. Auston, "Novel sources and detectors for coherent tunable narrow-band terahertz radiation in free space," $J$. Opt. Soc. Am. B, vol. 13, pp. 2783-2791, 1996.

[15] J. Palací and B. Vidal, "Tunable and reconfigurable narrow-band $\mathrm{THz}$ generation using photoconductive antennas and chirped-pulse mixing," in Proceedings of the International Topical Meeting on Microwave Photonics (Singapore, 2011), pp. 210-213.

[16] R. E. Saperstein, N. Alic, D. Panasenko, R. Rokitski and Y. Fainman, "Time-domain waveform processing by chromatic dispersion for temporal shaping of optical pulses," J. Opt. Soc. Am. B, vol. 22, pp. 2427-2436, 2005.

[17] H. Ju, S. Zhang, D. Lenstra, H. de Waardt, E. Tangdiongga, G. Khoe and H. Dorren, "SOA-based all-optical switch with subpicosecond full recovery," Opt. Express, vol. 13, pp. 942-947, 2005.

[18] A. J. Zilkie, J. Meier, M. Mojahedi, P. J. Poole, P. Barrios, D. Poitras, T. J. Rotter, C. Yang, A. Stintz, K. J. Malloy, P. W. E. Smith and J. S. Aitchison, "Carrier dynamics of quantum-dot, quantum-dash, and quantum-well semiconductor optical amplifiers operating at 1.55

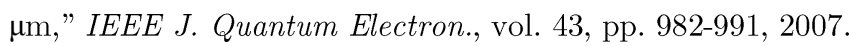


[19] A.S. Weling and T.F. Heinz, "Enhancement in the spectral irradiance of photoconducting terahertz emitters by chirped-pulse mixing," $J$. Opt. Soc. Am. B, vol. 16, pp. 1455-1467, 1999.

[20] R. Inohara, K. Nishimura, M. Tsurusawa and M. Usami, "Experimental analysis of cross-phase modulation and cross-gain modulation in SOA-injecting CW assist light," IEEE Photon. Technol. Lett., vol. 15, pp. 1192-1194, 2003.

[21] A. J. Lowery, "New dynamic semiconductor laser model based on the transmission line modeling method," IEE Proc. J. Optoelectron., vol. 134, pp. 281-289, 1987.

[22] A. I. Siahlo, A. T. Clausen, L. K. Oxenløwe, J. Seoane, K. S. Berg, Z. $\mathrm{Xu}$, J. Zeng and P. Jeppesen, "Phase modulation for postcompensation of dispersion in $160 \mathrm{~Gb} / \mathrm{s}$ systems," IEEE Photon. Technol. Lett., vol. 17, pp. 498-500, 2005. 



\title{
Paper F
}

\section{"SOA-based optical processing for terahertz time-domain spectroscopy"}

\author{
J. Palací, A. Bockelt and B. Vidal
}

Electron. Lett, Vol. 48, No. 10, pp. 593-594, May 2012 



\title{
SOA-based optical processing for terahertz time-domain spectroscopy
}

\author{
Jesús Palací, Alexander Bockelt and Borja Vidal \\ Nanophotonics Technology Center, \\ Universidad Politécnica de Valencia, Camino \\ de Vera s/n, 46022 Valencia, Spain
}

\begin{abstract}
All-fibre control of a terahertz time-domain spectroscopy system based on photoconductive antennas fed with optical sources in the telecom band is demonstrated. A semiconductor optical amplifier (SOA) is used to obtain continuously tunable delays on 100 fs optical pulses. Additionally, the modulation required to carry out lock-in detection can be simultaneously performed avoiding mechanical choppers or high-voltage alternate biasing of the transmitter antenna.
\end{abstract}

\section{Introduction}

Considerable efforts are being carried out to develop simpler terahertz $(\mathrm{THz})$ systems and ease the practical exploitation of radiation in this spectral region. Photoconductive antennas are commonly employed in both generation and detection of $\mathrm{THz}$ waves because of their flexibility and bandwidth. Ti:Sapphire lasers working at $800 \mathrm{~nm}$ are usually used since traditional photoconductive antennas made of low-temperaturegrown GaAs (LT-GaAs) require such photon energies. However, recent advances have led to the development of antennas fed at $1550 \mathrm{~nm}$ [1] allowing the use of cost-effective fibre lasers and the implementation of distributed architectures with reduced $\mathrm{THz}$ heads [2].

In this Letter we report on the processing of ultra-short optical pulses by means of a semiconductor optical amplifier (SOA) [3] with application in a classical terahertz time-domain spectroscopy (THzTDS) system fed with $1550 \mathrm{~nm}$ sources. The implementation of continuously tunable delays on the optical pulse is directly observed at $\mathrm{THz}$ frequencies showing a delay-bandwidth product of 10. Chopping of the optical signal, necessary to carry out the detection of the $\mathrm{THz}$ 
field, can be simultaneously performed through the modulation of the bias current of the SOA.

\section{Principle of operation}

When optical pulses with sufficient peak power are injected into a SOA, saturation occurs leading to changes in the pulse amplitude and phase [4]. In the case of pulses that have been previously chirped, their spectral components are differently affected by the SOA. If the pulse is recompressed after the SOA, an advanced or delayed version of the initial pulse is obtained depending on the sign of the initial chirp [5]. Since the pulse spectral shaping is related to the gain recovery time of the SOA and it is given by the amount of current-injected carriers, it is possible to control the temporal shift by tuning the SOA bias current. Fig. 1 sums up this process.

\section{Experimental results}

The terahertz processing system proposed is shown in Fig. 2. It is a conventional THz-TDS system where a SOA has been included to implement tunable delays. Ultra-short optical pulses with a full-width at half maximum duration of 100 fs delivered at a $50 \mathrm{MHz}$ repetition rate by a $1.55 \mathrm{\mu m}$ fibre mode-lock laser are driven to both the transmitter and the receiver photoconductive antennas (PCAs). Dispersion pre-compensation is required to obtain narrow pulses at the PCAs while minimizing nonlinear effects taking place during fibre transmission. An average power of $6 \mathrm{dBm}$ at the output of the laser was measured. At the transmitter branch pulses were additionally stretched up to $30 \mathrm{ps}$ before entering the SOA by means of $16 \mathrm{~m}$ of dispersion-compensating fibre (DCF) with an accumulated dispersion of $-1.66 \mathrm{ps} / \mathrm{nm}$. The optical power at the input of the SOA was set to $-8 \mathrm{dBm}$, enough to saturate the multi-quantum well SOA used in the experiment (Philips CQF871). Its transparency current was experimentally estimated to be around $12 \mathrm{~mA}$. The SOA-induced delay on the pulse is controlled by changing the SOA bias current. The pulses compress again by transmission through standard singlemode fibre (SSMF). The SSMF length was estimated to compensate for the accumulated second-order dispersion of the transmitter branch. A pair of erbium-doped fibre amplifiers (EDFAs) was employed to 
boost the optical power at the input of the PCAs up to $6 \mathrm{dBm}$. The transmitter PCA was biased with a continuous voltage of $18 \mathrm{~V}$. A computer controlled motorized optical delay line (MODL) has been included in the experimental setup to characterize the SOA generated time delays. Lock-in detection is carried out in order to obtain clean traces of the $\mathrm{THz}$ signal, meaning that chopping of the optical train of pulses is required. It is performed by means of a mechanical chopper at $1 \mathrm{kHz}$ which was placed in the $\mathrm{THz}$ path. An integration time of 30 ms was employed in the lock-in amplifier to obtain the traces. At the input of the transmitter and receiver PCAs pulses with an approximated duration of 300 and 200 fs were obtained, respectively. This is due to uncompensated third-order dispersion, which becomes the dominant source of degradation once the second-order dispersion is compensated.

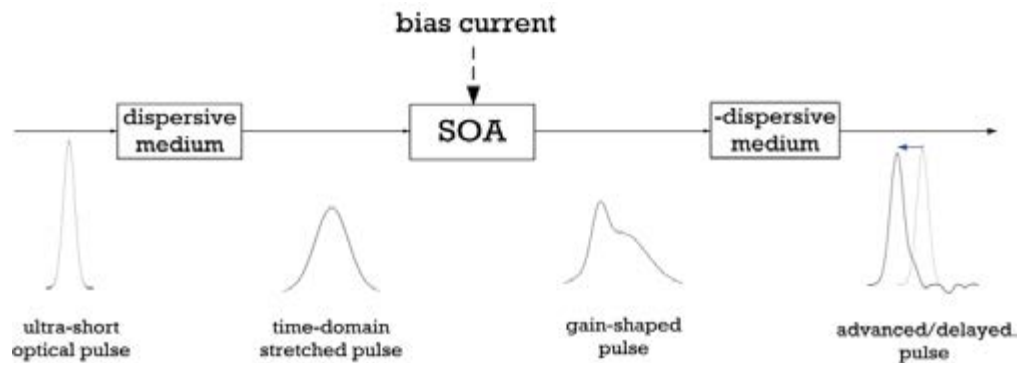

Figure 1: Concept of pulse delay/advance by means of SOA gain saturation

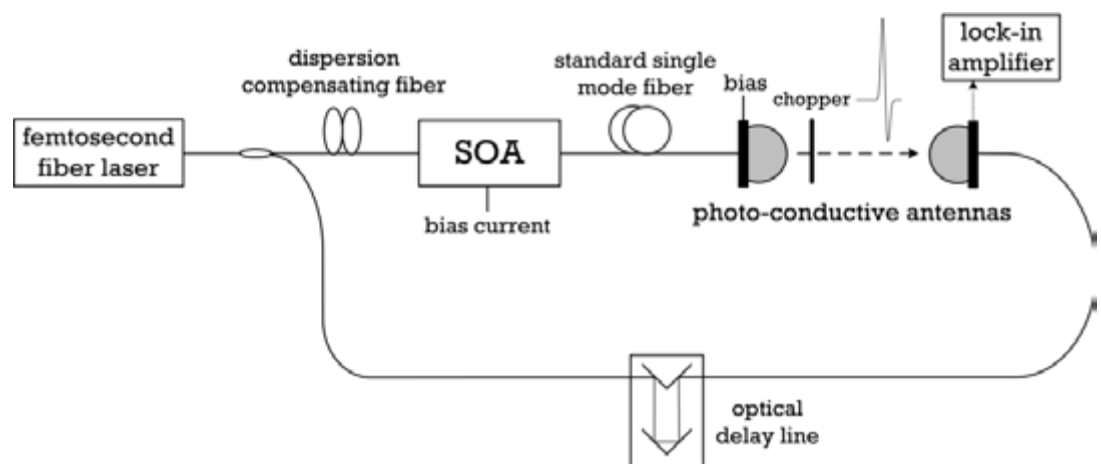

Figure 2: Experimental setup for all-fibre control of $\mathrm{THz}$ time-domain spectroscopy system. An optical delay line is employed to check the performance of the delayed pulses in the $\mathrm{THz}$ signal 


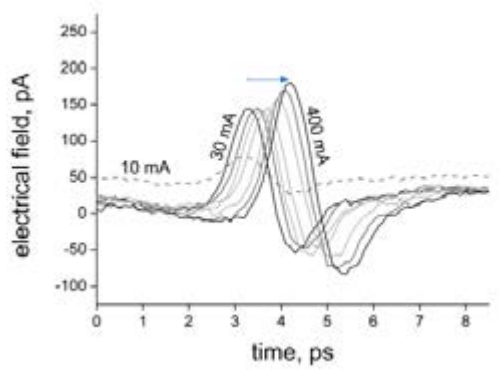

(a)

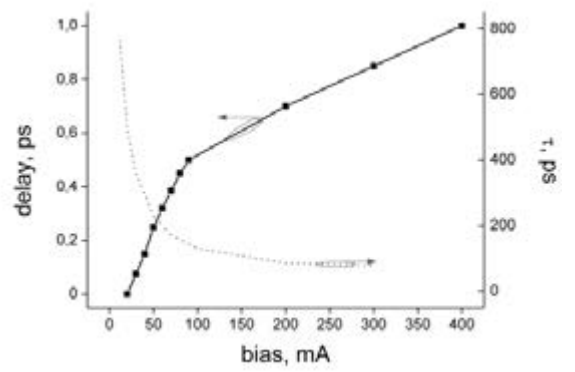

(b)

Figure 3: Experimental results. (a) Time-domain terahertz traces obtained against different SOA bias current values. (b) Delay and gain recovery time against SOA bias current.

The experimental measurements obtained are shown in Fig. 3a, where the delay induced in the pulse is clearly observed when changing the current injected to the SOA. The dashed line is for a current below transparency. For higher values the amplitude of the terahertz pulse is kept almost constant for currents up to $100 \mathrm{~mA}$ and then increases. All $\mathrm{THz}$ pulses exhibit a similar signal-to-noise ratio. Fig. $3 \mathrm{~b}$ shows the introduced delay with the bias current according to data in Fig. 3a. A delay-bandwidth product of 10 and a nonlinear relation between the delay and the bias current are observed. This behavior relates to the gain recovery time of the SOA and its dependency on the bias current. When an intense optical pulse arrives at the SOA, it gets saturated, reducing its gain. The time required for recovery depends on the number of carriers available and thus on the injected current. A simple model for the gain dynamics assuming it recovers to its initial state according to a first-order exponential decay with a time constant $\tau$ will be assumed as in [6]. To measure the SOA gain response a low-power optical carrier (probe) is allocated outside the bandwidth of the optical pulsed source (pump) and injected to the SOA. Its power must be as low as not to influence the SOA. This is checked in an optical spectrum analyzer by turning the probe on and off. The probe is spectrally filtered at the output of the SOA and then photo-detected, providing direct measurement of the gain changes originated by the chirped pulses. The obtained results are plotted in Fig. 3b. For low current values large variations in the recovery time 
originate from small increments in the bias current while for large currents small changes in the recovery time occur. Noticeable variations in the recovery time imply that the chirped pulse gets shaped in a different way leading to changes in the delay. It can be seen that large (small) increases in $\tau$ translate into large (small) increases for the delay. Moreover, larger delays and a more precise control on the pulse amplitude can be obtained using several SOAs in cascade [7].

When measuring time-domain $\mathrm{THz}$ pulses lock-in detection turns out to be necessary to improve the signal-to-noise ratio. Therefore, some kind of modulation must be applied to the pulses. This is usually performed using a mechanical chopper or applying a high-voltage lowfrequency modulation signal to the transmitter antenna. The SOA can also be used as an optical modulator. Since it is possible to saturate its gain even for low injected currents a low-cost signal generator could be employed. Furthermore, both the optical modulation and pulse delay can be performed simultaneously. By applying a square signal whose amplitude levels are $0 \mathrm{~mA}$ and $\mathrm{I}_{\text {mod }}$ no pulse is transmitted for the low level while for the high one a time delay given by the amount of current injected applies to the pulse. Fig. 4 shows results for different values of $\mathrm{I}_{\text {mod }}$ and a frequency of $1 \mathrm{kHz}$. Clean traces as well as similar spectra are observed with no severe penalties compared with previous results. Although the same low frequency was employed for comparison purposes the electrical port of the SOA can be modulated up to several $\mathrm{MHz}$, potentially reducing the measurement time required to carry out $\mathrm{THz}$ measurements.

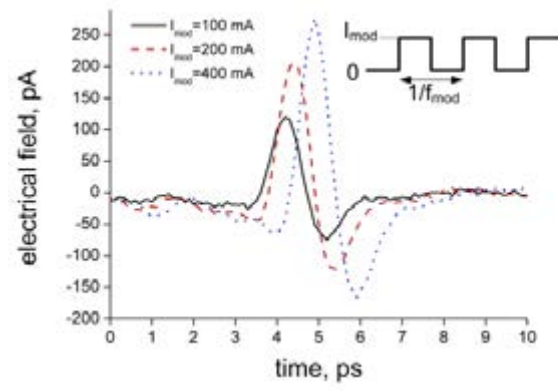

(a)

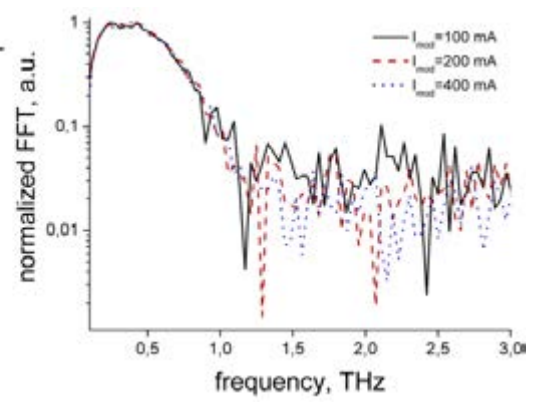

(b)

Figure 4: Experimental results employing SOA as modulator. (a) Timedomain terahertz traces for different SOA modulation current values. (b) Fourier transform of previous pulses. 


\section{Conclusion}

In this Letter the use of SOAs to control THz-TDS systems has been studied. A single device allows the implementation of time delay and signal modulation. A delay-bandwidth product of 10 for 100 fs pulses has been obtained. Larger time delays can be obtained by using more than one SOA or by further chirping of the optical pulse at its input. The complexity of the system is reduced since no mechanical parts or high-voltage amplifiers for the modulation of the photoconductive antenna bias are required.

\section{References}

[1] M. Kamakura, A. Takasato, J. Kitagawa, and Y. Kadoya "Generation and detection of $\mathrm{THz}$ waves using InGaAs photoconductive antenna with $1.55 \mu \mathrm{m}$ excitation," Conf. on Lasers and Electro-Optics, Long Beach, CA, USA, 2006.

[2] J. Palací and B. Vidal, "Distributed $\mathrm{THz}$ transmitter/receiver based on a $1.5 \mu \mathrm{m}$ fiber link," 36th Int. Conf. on Infrared, Millimeter and Terahertz Waves, Houston, TX, USA, 2011.

[3] M.J. Conelly, Semiconductor optical amplifiers, Boston, MA: Kluwer Academic, 2002.

[4] G. P. Agrawal and N. A. Olsson, "Self-phase modulation and spectral broadening of optical pulses in semiconductor laser amplifiers," IEEE J. Quantum Electron., vol. 25, no. 11, pp. 2297-2306, 1989.

[5] F. G. Sedgwick, B. Pesala, A. V. Uskov, and C. J. Chang-Hasnain, "Chirp-enhanced fast light in semiconductor optical amplifiers," Opt. Express, vol. 15, no. 26, pp. 17631-17638, 2007.

[6] R. Inohara, K. Nishimura, M. Tsurusawa, and M. Usami, "Experimental analysis of cross-phase modulation and cross-gain modulation in SOA-injecting CW assist light," IEEE Photonics Technol. Lett., vol. 15, no. 9, pp. 1192-1194, 2003.

[7] B. Pesala, F. Sedgwick, A. V. Uskov, and C. Chang-Hasnain, "Electrically tunable fast light at $\mathrm{THz}$ bandwidth using cascaded semiconductor optical amplifiers," Opt. Express, vol. 15, no. 24, pp. 15863-15867, 2007. 


\title{
Paper G
}

"Tunable optical delay line based on single sideband suppressed-carrier modulation"

\author{
J. Palací and B. Vidal
}

IEEE Photon. Technol. Lett., Vol. 25, No. 1, pp. 43-46, January 2013 



\title{
Tunable optical delay line based on single-sideband suppressed-carrier modulation
}

\author{
Jesús Palací and Borja Vidal
}

\author{
Nanophotonics Technology Center, \\ Universidad Politécnica de Valencia, Camino \\ de Vera s/n, 46022 Valencia, Spain
}

\begin{abstract}
A technique to electrically control the time shift of ultra-short optical pulses without the generation of replicas is demonstrated. It is based on frequency-to-time conversion and single-sideband suppressed-carrier modulation of the optical spectrum. Pulses are advanced or delayed according to the modulated sideband. The time shift depends on the intensity of the frequency-to-time conversion and the frequency of the electro-optical modulation. The technique can be applied to pulses with any duration although it is susceptible to pulse widening due to the combined effect of nonlinear frequency-totime conversion and electro-optical modulation. Experimental results show dynamic shifting of ultra-short optical pulses in a range of $100 \mathrm{ps}$ at the expenses of widening originating from the third-order dispersion of the fiber.
\end{abstract}

\section{Introduction}

Controlling the speed at which light propagates provides important utilities in several fields. It allows increased light-matter interaction for nonlinear applications and is essential for the future development of optical memories [1-2].

In particular, the control of the speed of optical pulses has attracted considerable attention recently due to its applicability in fields such as high-speed communications and optical sampling. Several solutions based on non-linear effects as stimulated Brillouin or Raman scattering [3-4] and self-phase modulation in optical fiber [5] have been proposed. While the former schemes provide small delays the latter provides much larger ones at the expenses of pulse widening. Another solution relies on gain saturation in a semiconductor optical amplifier [6], which however shows small delays with only a slight degradation of the pulse width. 
Schemes based on electro-optical modulation of the time-stretched optical spectrum have recently been reported [7-9]. In [7] a ramp-type signal feeding an electro-optical phase modulator introduced a timedependent phase shift onto the previously chirped pulses, which translated into a time delay once the pulses were recompressed. The spectrum was time-dispersed and recompressed by means of several chirped fiber Bragg gratings (CFBGs). The use of CFBG although feasible for picosecond pulses, becomes quite difficult for shorter pulses due to their large bandwidth. In [10], frequency-to-time conversion was performed by means of standard single-mode fiber (SSMF) and the amplitude modulation of the optical spectrum. This technique generates several pulses [11] and a second stage is required to filter the pulse of interest, which increases the complexity of the system and requires synchronization.

In this letter we propose the implementation of a tunable all-fiber optical delay line by means of the single-sideband suppressed-carrier (SSB-SC) modulation of the time-decomposed optical spectrum. Only a single delayed pulse is generated, thus no additional processing is required.

\section{Principle of operation}

The principle of operation is detailed in Fig. 1. Optical pulses generated in a mode-locked laser (MLL) are injected into a dispersive device which performs frequency-to-time conversion because the different spectral components propagate at different group velocities. The stretched pulses are then modulated by means of an electrooptical modulator (EOM) before the opposite dispersion is applied to the pulses and the domain conversion is reverted (Fig. 1 (a)). SSB-SC modulation introduces a delay on the single sideband that depends on the local oscillator (LO) frequency while no additional pulses are generated. The process can be compared with the external modulation of a continuous-wave (CW) laser as shown in Fig. 1 (b). The main difference is the domain conversion performed before the electrooptical modulation, which leads to the generation of the additional components in the time domain instead of appearing in the spectral one $[12]$. 


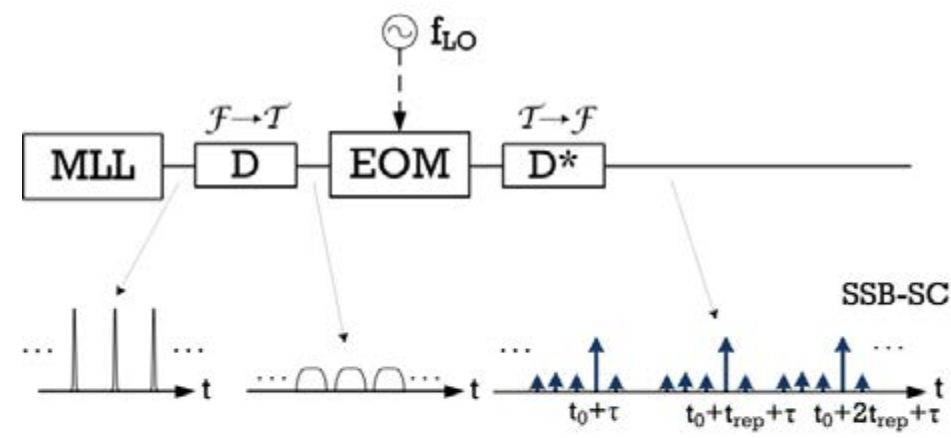

(a)

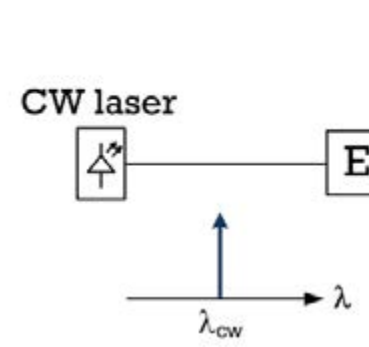

\section{$\mathrm{f}_{\mathrm{LO}}$}

EOM

(b)

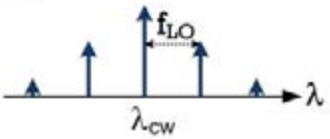

Fig. 1.- (a) External modulation of a mode-locked laser after frequency-to-time conversion. The signal is time-to-frequency converted after being modulated and the result shown for SSB-SC modulation. (b) External modulation of a single-mode laser.

The frequency-to-time conversion is performed using DCF, while SSMF recompresses the spectrum in time. The spectrum at the output of the DCF considering up to the third term of the expansion of the mode-propagation constant, $\beta$, in Taylor series, is

$$
S_{D C F}(\omega)=S_{f s F L}(\omega) \exp \left(-j\left(\frac{\beta_{2}{ }^{D C F}}{2} \omega^{2}+\frac{\beta_{3}^{D C F}}{6} \omega^{3}\right) L^{D C F}\right)
$$

$\omega$ being frequency, $S_{f_{s} F}(\omega)$ the optical spectrum of the femtosecond laser, $L^{D C F}$ the DCF length, and $\beta_{2}$ and $\beta_{3}$ the second- and third-order dispersion parameters, SOD and TOD, respectively.

This signal is injected into the DP-EOM and its spectral components recompressed by the SSMF. The resultant spectrum for the delayed pulse is 


$$
\begin{aligned}
& S_{\text {out }}(\omega) \propto S_{f S F L}\left(\omega-\omega_{L O}\right) \times \exp \left(\frac{j}{6}\left(3 \beta_{2}{ }^{D C F}+\beta_{3}{ }^{D C F} \omega_{L O}\right) L^{D C F} \omega_{L O}{ }^{2}\right) \\
& \times \exp \left(-j\left(\beta_{2}{ }^{D C F}+\frac{1}{2} \beta_{3}^{D C F} \omega_{L O}\right) L^{D C F} \omega_{L O} \omega\right) \\
& \times \exp \left(-\frac{j}{2}\left(\beta_{2}{ }^{D C F} L^{D C F}+\beta_{2}{ }^{S S M F} L^{S S M F}+\beta_{3}{ }^{D C F} L^{D C F} \omega_{L O}\right) \omega^{2}\right) \\
& \times \exp \left(-\frac{j}{6}\left(\beta_{3}{ }^{D C F} L^{D C F}+\beta_{3}^{S S M F} L^{S S M F}\right) \omega^{3}\right)
\end{aligned}
$$

where $\omega_{L O}$ accounts for the LO frequency and $L^{S S M F}$ and $\beta^{S S M F}$ correspond to length and dispersion parameters of the standard fiber.

The spectrum of the pulse is the convolution of the transformlimited source spectrum with different terms. The first exponential does not depend on $\omega$ therefore not affecting the pulse shape. The second exponential corresponds to the temporal shift of the pulse. Its value depends on the parameters of the DCF and the LO frequency as

$$
\tau=\left(\beta_{2}{ }^{D C F} \omega_{L O}+\frac{1}{2} \beta_{3}{ }^{D C F} \omega_{L O}^{2}\right) L^{D C F}
$$

Although the dependence of the delay on the modulation frequency is quadratic it can be considered linear for the dispersion parameters and modulation frequencies considered in this work. The third exponential term of (2) introduces a quadratic phase change in the spectrum and accounts for the pulse widening introduced by the SOD of DCF and SSMF and the accumulated TOD of the DCF multiplied for the modulation frequency. Although the first two components may get cancelled as long as the accumulated SOD of both fiber links presents the same magnitude and different sign, the last term depends on the LO frequency. Thus, it cannot be compensated for the whole set of delayed pulses unless controllable dispersion compensating devices such as spatial gratings of even pulse shapers are employed. Its effect is expected to limit the performance of the system, especially for large delays. The last term of (2) accounts for cubic changes in the phase of the spectrum, affecting the pulse shape through uncompensated TOD.

Simultaneous compensation of both SOD and TOD is difficult because it requires identical SOD/TOD ratios between the compensating and compensated fibers. Thus, the most harmful dispersion term is often compensated. Assuming unchirped Gaussian 
pulses and perfect SOD compensation the full width at half maximum (FWHM) duration of the time shifted pulses can be expressed as a function of the parameters of the system as follows [13]

$$
T_{1}=T_{0} \sqrt{1+\left(\frac{\beta_{3}{ }^{D C F} L^{D C F} \omega_{L O}}{T_{0}^{2}}\right)^{2}+\left(\frac{\beta_{3}{ }^{D C F} L^{D C F}+\beta_{3}{ }^{S S M F} L^{S S M F}}{T_{0}^{3}}\right)^{2}}
$$

As shown in (4) pulses are widened due to uncompensated TOD and the nonlinear response of the frequency-to-time conversion stage whose importance raises for increasing modulation frequencies. The degradation introduced could be dynamically compensated by phase modulation of the chirped pulses [14]. According to (2) there is a change in the carrier frequency due to the time shift. Additional modulation at the output of the system could bring the signal back to its original spectral band.

\section{Experimental results}

The experimental setup is shown in Fig. 2. Gaussian pulses with a FWHM of 100 fs are generated in a femtosecond fiber laser (fs FL). The repetition rate is $50 \mathrm{MHz}$ and the average power is $5 \mathrm{dBm}$, which is low enough to minimize the non-linear effects taking place during fiber propagation. These pulses are time-stretched up to 5 ns by means of $2.7 \mathrm{~km}$ of DCF with dispersion of $-160 \mathrm{ps} / \mathrm{km} \cdot \mathrm{nm}$ and a dispersion slope of $-0.2 \mathrm{ps} / \mathrm{km} \cdot \mathrm{nm} 2$. They are injected into a $10-\mathrm{GHz}$ DP-MZM which is fed by a frequency tunable microwave generator. A microwave $\pi 2$ hybrid suitable for the $2-18 \mathrm{GHz}$ band is also employed. By properly adjusting the bias points of the DP-MZM a SSB-SC modulation is applied to the chirped pulses. The modulated pulses are recompressed by means of SSMF. The shape and introduced delay are observed in an optical autocorrelator (OA) and a digital communications analyzer (DCA). Amplification was introduced when necessary by means of Er-doped fiber amplifiers (EDFAs).

Temperature changes on the long fiber reels leads to noticeable delay changes due to thermal expansion/contraction. According to the coefficient of thermal expansion of the silica at room temperature $(0.5$ $\mathrm{ppm} / \mathrm{K})$, the additional advancement/delay is $75 \mathrm{ps} / \mathrm{K}$, in good agreement with the observations. This problem was solved by 
introducing several optical circulators (OCs) in the system in such a way that the fs FL output went through an optical splitter (OS) and was used as the DCA trigger after passing through both SSMF and DCF reels and being photo-detected (PD). This solution provides stability to interferometry setups such as $\mathrm{THz}$ time-domain spectroscopy systems [15].

Fig. 3 shows the results obtained. The microwave hybrid was removed and measurements for DSB modulation obtained as shown in Fig. 3 (a). The $\mathrm{f}_{\mathrm{LO}}$ swept the range $3-15 \mathrm{GHz}$ in $2 \mathrm{GHz}$ steps. The traces of Fig. 3 were obtained in an $80-\mathrm{GHz}$ bandwidth DCA, thus the real pulse width is not represented. The inset of Fig. 3 (a) shows the advance/delay $(\tau)$ introduced on the sidebands as a function of LO frequency. The inset of Fig. 3(b) shows that carrier-suppressed modulation was obtained for both the lower- (LSB) and uppersidebands (USB). An extinction ratio of $20 \mathrm{~dB}$ with respect to the optical carrier and removed sideband was obtained. Fig. 3 shows that single advanced/delayed pulses can be achieved when SSB-SC modulation is performed. In particular, Fig.3 (b) shows advanced and delayed pulses for LSB-SC and USB-SC modulations, respectively. The lower amplitude of the pulses with longer delays is due to the poor response of the modulator used in the experiments for high frequencies.

Once the operating principle has been demonstrated, autocorrelation traces and delay characterization as a function of the LO frequency were performed. Fig. 4 (a) shows experimental results for the delay. It fits the theoretical response, which can be considered linear with $\beta_{2}^{D C F} \cdot L^{D C F}$ slope in terms of the LO angular frequency as predicted by theory.

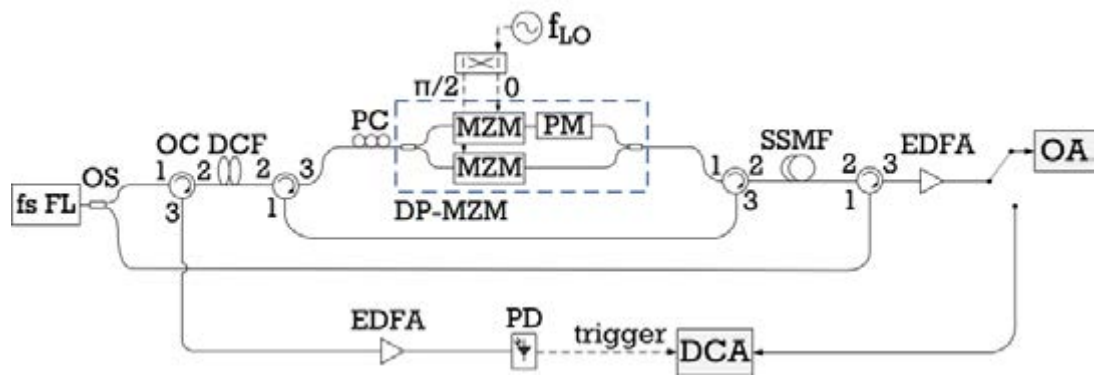

Fig. 2.- Experimental setup. 


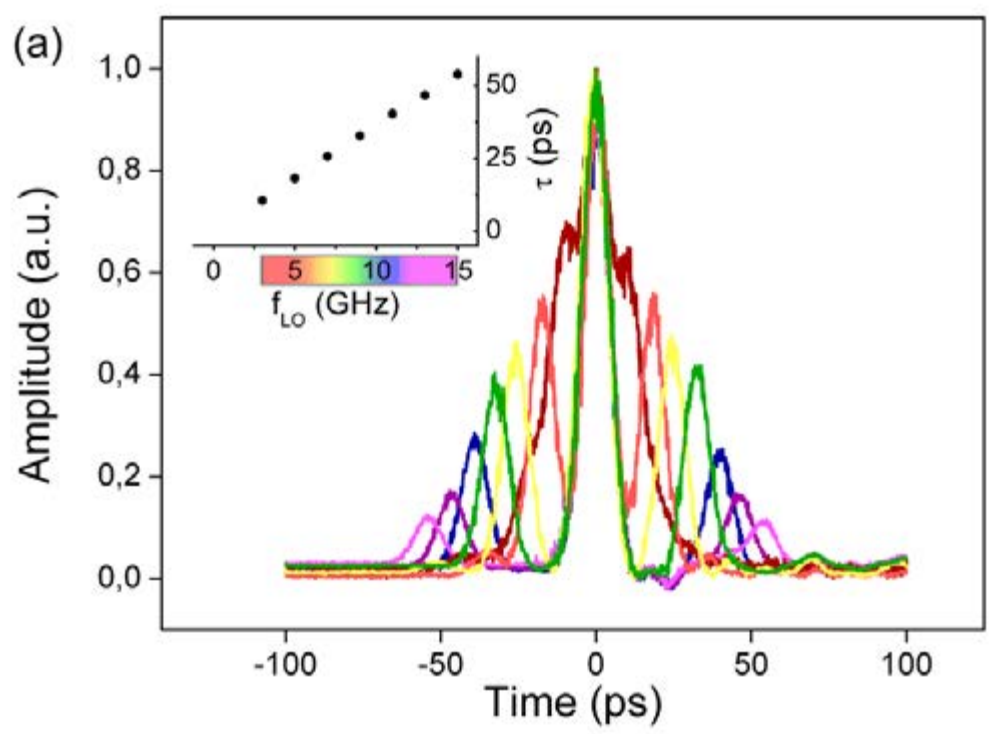

(b)

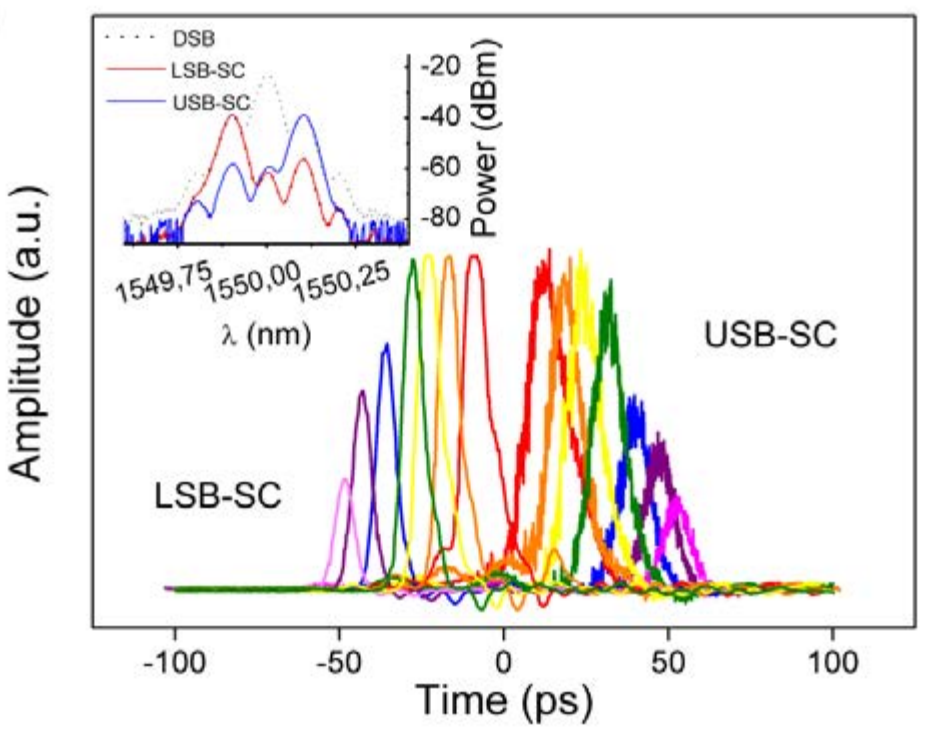

Fig. 3.- (a) Optical pulses generated after DSB modulation. Inset: time difference between the main and secondary pulses as a function of the modulation frequency. (b) Optical pulses generated after LSB-SC and USB-SC modulation. Inset: optical spectra for DSB and SSB-SC modulation of a CW laser. 

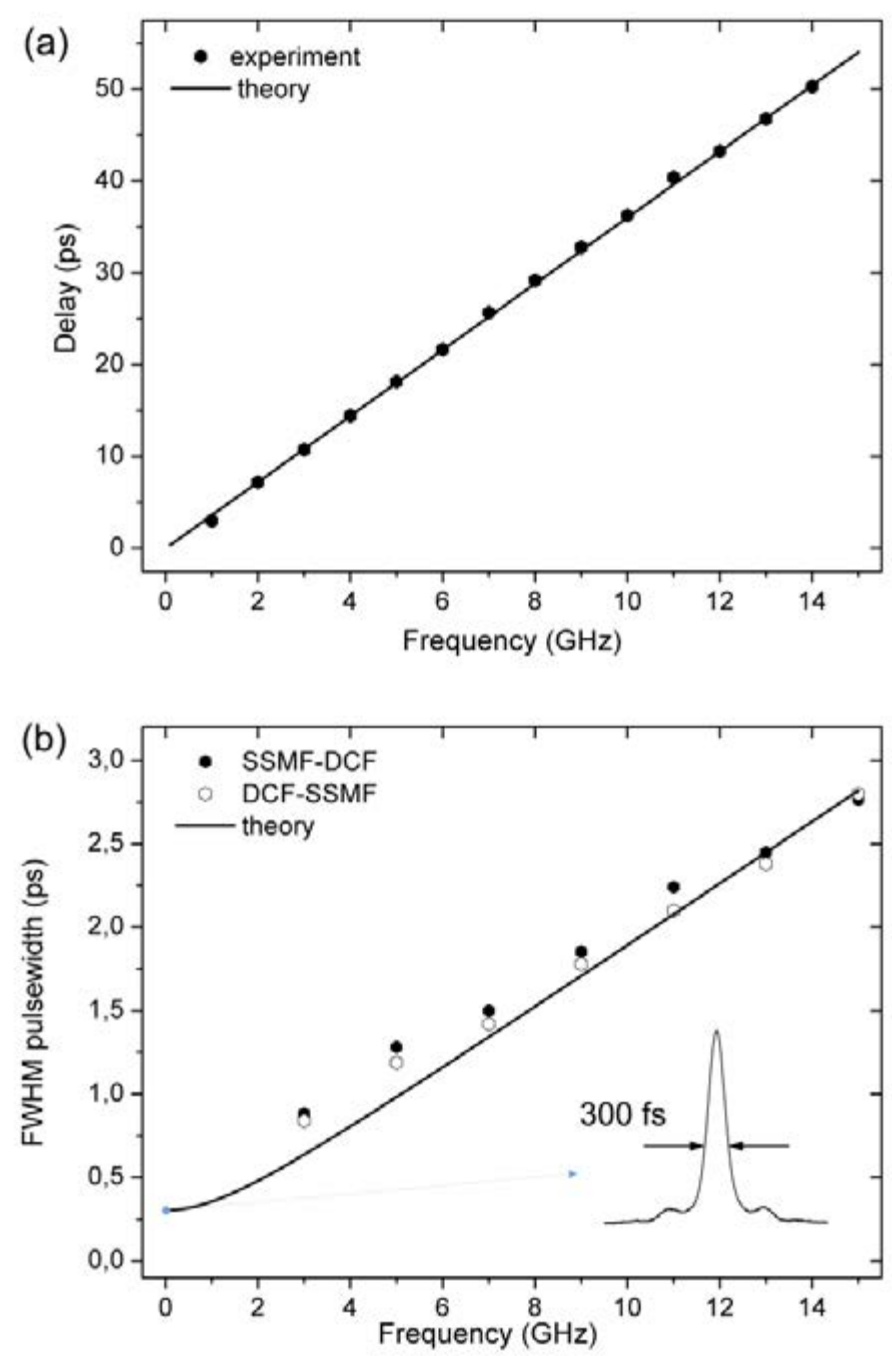

Fig. 4.- (a) Experimental and theoretical values for the delay as a function of the local oscillator frequency. (b) Theoretical full-width at half maximum pulse-width values for the delayed pulse as well as experimental results.

The coefficient of determination for a linear fit of the experimental results was greater than 0.99 . Fig. 4 (b) shows the experimental measurement of the width of the advanced/delayed pulses when using the DCF and SSMF as frequency-to-time converters. The width of the pulses is estimated by means of optical autocorrelation and assuming 
Gaussian-shaped pulses. A FWHM of 300 fs with small side-lobes originating from the uncompensated TOD is obtained for the pulses with no modulation. To compensate for TOD the ratio between the magnitudes of the $\beta_{3}$ parameters is similar to the one for $\beta_{2}$. The theoretical degradation of the pulses shown in Fig. 4 (b) is given by (4). Good agreement is observed between theory and experiments. Although the duration of the pulse has been provided at several parts of the system, it could be estimated at any point by numerically solving the equation for propagation of pulses through optical fiber [13]. A time shifting of the original $100-$ fs up to 100 ps imply a delaybandwidth product of the system of 1000 although different degradations widen the output pulses up to the ps range. Enhanced performance could be obtained by means of additional processing techniques [14] or a more linear frequency-to-time converter.

Dependence of the pulse duration at the output of the system on the LO frequency limits the maximum data rate that can be transmitted. For the experiments performed rates exceeding 300 Gsymbol/s can be transmitted without inter-symbol interference.

\section{Conclusion}

It has been shown that single-sideband suppressed-carrier modulation of the spectrum of ultra-short pulses is an efficient technique to obtain single delayed pulses with a simple setup. Femtosecond pulses have been advanced and delayed up to 100 ps by means of frequency-totime conversion in optical fiber. Since the technique is not bandwidth limited it can be applied to pulses with arbitrary duration, being its performance limited only by the linearity of the frequency-to-time converter and the residual dispersion.

\section{References}

[1] L. V. Hau, S. E. Harris, Zachary Dutton, and Cyrus H. Behroozi. "Light speed reduction to 17 metres per second in an ultracold atomic gas," Nature, vol. 397, pp. 594-598, Feb. 1999.

[2] Thomas F. Krauss, "Why do we need slow light?," Nature Photonics, vol. 2, pp. 448-450, Aug. 2008.

[3] K. Y. Song, M. G. Herráez, and L. Thévenaz, "Observation of pulse delaying and advancement in optical fibers using stimulated Brillouin scattering," Opt. Express, vol. 13, no. 1, pp. 82-88, Jan. 2005. 
[4] J. E. Sharping, Y. Okawachi, and A. L. Gaeta, "Wide bandwidth slow light using a Raman fiber amplifier," Opt. Express, vol. 13, no. 16, pp. 6092-6098, Aug. 2005.

[5] Y. Okawachi, J. E. Sharping, C. Xu, and A. L. Gaeta, "Large tunable optical delays via self-phase modulation and dispersion," Opt. Express, vol. 14, no. 25, pp. 12022-12027, Dec. 2006.

[6] B. Pesala, F. Sedgwick, A. V. Uskov, and C. Chang-Hasnain, "Electrically tunable fast light at $\mathrm{THz}$ bandwidth using cascaded semiconductor optical amplifiers," Opt. Express, vol. 15, no. 24, pp. 15863-15867, Nov. 2007.

[7] M. Xin, F. Yin, C. Lei, M. Chen, H. Chen, C. Tang, and S. Xie, "Broadband tunable optical delay based on real-time Fourier transformation and ramp-type phase modulation," Opt. Lett., vol. 35, no. 10, pp. 1596-1598, May 2010.

[8] K. Jamshidi, A. Wiatrek, C. Bersch, G. Onishchukov, G. Leuchs, C.A. Bunge and T. Schneider, "Very large, tunable, positive and negative group delay for high-bandwidth signals," in European Conference on Communication. ECOC 2010, Turin, Italy, Sep 2010.

[9] K. Jamshidi, S. Meister, B. A. Franke, O. Dyatlova, A. Al-saadi, U. Woggon, H. J. Eichler, and T. Schneider, "Compact electrically tunable delay generator on silicon," in Conference on Lasers and Electro-Optics. CLEO 2012, San Jose, CA, USA, May 2012.

[10] K. Jamshidi, A. Wiatrek, C. Bersch, G. Onishchukov, G. Leuchs, and T. Schneider, "Widely tunable optical delay generator," Opt. Lett., vol. 35, no. 21, pp. 3592-3594, Nov. 2010.

[11] S. Thomas, A. Malacarne, F. Fresi, L. Potì, and J. Azaña, "Fiberbased programmable picosecond optical pulse shaper," J. Lightw. Technol., vol. 28, no. 12, pp. 1832-1843, June 2010.

[12] R. E. Saperstein, N. Alic, D. Panasenko, R. Rokitski, and Y. Fainman, "Time-domain waveform processing by chromatic dispersion for temporal shaping of optical pulses," J. Opt. Soc. Am. B, vol. 22, no. 11, pp. 2427-2436, Nov. 2005.

[13] G. P. Agrawal, Nonlinear Fiber Optic, 4th ed. (Academic Press, 2006).

[14] K. Jamshidi, S. Meister, B. Franke, A. AlSaadi, S. Kupijai, and T. Schneider, "An integratable electrically tunable dispersion trimming," 
in Integrated Photonics Research, Silicon and Nanophotonics. IPRSN 2012, Colorado Springs, CO, USA, June 2012.

[15] P. U. Jepsen, R. H. Jacobsen, and S. R. Keiding, "Generation and detection of terahertz pulses from biased semiconductor antennas," $J$. Opt. Soc. Am. B, vol. 13, no. 11, pp. 2424-2436, Nov. 1996. 
